\title{
Carbon Nanotube Synthesis and Organization
}

\author{
Ernesto Joselevich ${ }^{1}$, Hongjie Dai ${ }^{2}$, Jie Liu ${ }^{3}$, Kenji Hata ${ }^{4}$, \\ and Alan H. Windle ${ }^{5}$
}

1 Department of Materials and Interfaces, Weizmann Institute of Science, Rehovot 76100, Israel ernesto.joselevich@weizmann.ac.il

2 Department of Chemistry, Stanford University Stanford, CA 94305, USA hdai10stanford.edu

3 Department of Chemistry, Duke University Durham, NC 27708, USA

\section{j.liu@duke.edu}

4 Research Center for Advanced Carbon Materials, National Institute of Advanced Industrial Science and Technology (AIST) 1-1-1 Higashi, Tsukuba, Ibaraki 305-8565, Japan kenji-hata@aist.go.jp

5 Department of Materials Science and Metallurgy, University of Cambridge Pembroke Street, Cambridge CB2 3QZ, UK

ahw1@cam.ac.uk

\begin{abstract}
The synthesis, sorting and organization of carbon nanotubes are major challenges toward future applications. This chapter reviews recent advances in these topics, addressing both the bulk production and processing of carbon nanotubes, and their organization into ordered structures, such as fibers, and aligned arrays on surfaces. The bulk synthetic methods are reviewed with emphasis on the current advances toward mass production and selective synthesis. New approaches for the sorting of carbon nanotubes by structure and properties are described in the context of the specific physical or chemical interactions at play, and referring to the characterization methods described in the contribution by Jorio et al. Recent advances in the organization of carbon nanotubes into fibers are reviewed, including methods based on spinning from solution, from dry forests, and directly from the gas phase during growth. The organization of carbon nanotubes on surfaces, as a critical prerequisite toward future applications in nanoelectronics, is reviewed with particular emphasis given to the synthesis of both vertically and horizontally aligned arrays. Vertically aligned growth has been recently boosted by the development of highly efficient catalytic processes. Horizontally aligned growth on surfaces can yield a whole new array of carbon-nanotube patterns, with interesting physical properties and potential applications. Different mechanisms of horizontally aligned growth include field- and flow-directed growth, as well as recently developed methods of surface-directed growth on single-crystal substrates by epitaxial approaches. The proposed mechanisms pertinent to each technique are discussed throughout this review, as well as their potential applications and critical aspects toward future progress.
\end{abstract}




\section{Introduction}

Carbon nanotubes can have different individual structures, morphologies and properties, as well as different collective arrangements and emerging properties, all of which are determined by the method of preparation and further processing. Hence, a wide variety of synthetic methods have been developed to produce the desired materials and properties for specific scientific studies or technological applications. The first growth of high-quality and milligram quantities of multiwall carbon nanotubes (MWNTs) [1] and single-wall carbon nanotubes (SWNTs) [2,3] marked important milestones that enabled the study of the intrinsic properties of nanotubes. Chemical vapor deposition (CVD) for high-quality SWNTs [4-6] further opened up new routes for controlled synthesis and device integration. It is clear that future developments in nanotube-based science and technology will continue to rely on the further improved highly controlled synthesis of nanotubes. Some of the longstanding problems in the nanotube area are due to the lack of control in the synthesis and chemical processing of SWNTs, in chirality control that determines whether a nanotube is metallic or semiconducting, in diameter control that determines the bandgap of a semiconducting SWNT, and in the placement and orientation control on large substrates that is needed for scalable production of nanotube electronics and other devices. Currently, there are four main challenges in the field of nanotube synthesis: 1. Mass production, i.e., the development of low-cost, large-scale processes for the synthesis of high-quality nanotubes, including SWNTs; 2. Selective production, i.e., control over the structure and electronic properties of the produced nanotubes; 3. Organization, i.e., control over the location and orientation of the produced nanotubes on a flat substrate; and 4. Mechanism, i.e., the development of a thorough understanding of the processes of nanotube growth. For applications such as composites and hydrogen storage, it is desired to obtain high-quality nanotubes at the kilogram or ton level using bulk-growth methods that are simple, efficient and inexpensive. For devices such as nanotube-based electronics, which require highly organized arrays, scaleup will unavoidably rely on self-assembly techniques or controlled growth strategies on surfaces combined with microfabrication techniques.

In this review, we present an overview of the current state-of-the-art methods and understanding in the synthesis of carbon nanotubes, following this rational order from bulk production to organized production, namely from methods involving large amounts of material and a low level of organization, to methods yielding higher levels of organization in smaller amounts. Thus, we start with bulk production methods (Sect. 2), with emphasis on mass production and selective synthesis, followed by purification (Sect. 3) and sorting (Sect. 4). Then we describe current methods for the production of nanotubes organized into fibers (Sect. 5) and into vertical and horizontal arrays on surfaces (Sect. 6), the latter representing the highest level of organization. Relevant mechanisms are briefly referred to throughout this re- 
view, and the general perspectives of nanotube synthesis are summarized in the last section (Sect. 7). For the synthesis of doped carbon nanotubes and double-walled carbon nanotubes, see contributions by Terrones et al. and Pfeiffer et al., respectively.

\section{Bulk Production Methods}

The preparation of high-quality carbon nanotubes with high yield has been the goal of many research endeavors. So far, arc discharge, laser ablation, and chemical vapor deposition (CVD) are the three main methods for SWNT production. In the following subsections, we discuss the various methods for the bulk synthesis of carbon nanotubes, first describing their general principles, and then focusing specifically on mass production and selective synthesis. In addition, some of the recent methods of vertical growth on surfaces (Sect. 6.1) can also yield bulk quantities of carbon nanotubes.

\subsection{Arc Discharge and Laser Vaporization}

Arc discharge and laser ablation were the first methods that allowed synthesis of SWNTs in relatively large (gram) amounts [7]. Both methods involve the condensation of hot gaseous carbon atoms generated from the evaporation of solid carbon. In 1992, a breakthrough in MWNT growth by arc discharge was first achieved by Ebbesen and Ajayan who demonstrated growth and purification of high-quality MWNTs at the gram level [1]. For the growth of single-wall tubes, a metal catalyst is needed in the arc-discharge system. The first success in producing substantial amounts of SWNTs by arc discharge was achieved by Bethune and coworkers in 1993 [2]. The growth of highquality SWNTs at the 1-10 g scale was achieved by Smalley and coworkers using a laser-ablation (laser oven) method [3]. Nevertheless, the equipment requirements and the large amount of energy consumed by these methods make them less favorable for nanotube production. With the arc and laser methods, only powdered samples with nanotubes tangled into bundles can be produced. Controlled synthesis on substrates with ordered nanotube structures has not been possible by these methods.

\subsection{Chemical Vapor Deposition (CVD)}

The CVD method involves the decomposition of a gaseous or volatile compound of carbon, catalyzed by metallic nanoparticles, which also serve as nucleation sites for the initiation of carbon-nanotube growth. This method, which can be easily scaled up to industrial production levels, has become the most important commercial method for SWNT production [7-9]. Chemical vapor deposition is the term used to describe heterogeneous reactions 
in which both solid and volatile products are formed from a volatile precursor through chemical reactions, and the solid products are deposited on a substrate. Both MWNT and SWNT synthesis have been well developed using CVD. Another advantage of CVD methods is that they allow more control over the morphology and structure of the produced nanotubes. With the CVD methods, one can produce well-separated individual nanotubes either supported on flat substrates or suspended across trenches [7-9]. These nanotubes can be directly used to fabricate nanoscale electronics.

Over the last ten years, several methods have been developed that have the potential for industrial-scale preparation of nanotubes. All of them are based on CVD methods. Among these methods, five different approaches have been shown to be the most promising: methane CVD, HiPCO, CO CVD, alcohol CVD and PECVD (plasma-enhanced CVD). Methane CVD was developed by Dai's group at Stanford; they first reported the synthesis of bulk amounts of SWNTs by CVD from methane at $900^{\circ} \mathrm{C}[5,10,11]$. Su et al. significantly improved the yield of this method using $\mathrm{Al}_{2} \mathrm{O}_{3}$ aerogels impregnated with Fe/Mo nanoparticles as a catalyst [12]. Several groups used other hydrocarbons and catalysts to prepare SWNTs. For example, Hafner et al. prepared SWNTs using an extremely small amount of $\mathrm{C}_{2} \mathrm{H}_{4}$ diluted by $\mathrm{Ar}$ and an $\mathrm{Fe} / \mathrm{Mo}$ bimetallic catalyst with an $\mathrm{Al}_{2} \mathrm{O}_{3}$ support. Both single- and double-wall nanotubes were observed for reaction temperatures from $700{ }^{\circ} \mathrm{C}$ to $750{ }^{\circ} \mathrm{C}[6]$.

HiPCO, which stands for high-pressure catalytic decomposition of carbon monoxide [13], is a method for the preparation of SWNTs using high-pressure CO as the carbon source. The catalysts used in a HiPCO process are formed in the gas phase from a volatile organometallic catalyst precursor introduced into the reactor. The organometallic species decompose at high temperature, forming metal clusters on which SWNTs nucleate and grow. The HiPCO process was originally developed by Smalley's research group at Rice University. Currently, the HiPCO process is the only process that can make SWNTs on a kilogram per day scale. CO was actually the first feed gas used for the growth of SWNTs. Dai et al. performed the first CVD synthesis of SWNTs by Mo-catalyzed disproportionation of CO at $1200^{\circ} \mathrm{C}$ in 1996 [4]. It was reported that most of the resulting SWNTs had catalytic particles attached to the ends, indicating that the growth of SWNTs was catalyzed by preformed nanoparticles. The use of $\mathrm{CO}$ as a feed gas does offer certain advantages over hydrocarbons. Compared with samples made using the same catalyst and methane, the amount of amorphous carbon can be reduced. An important advance in the CO CVD method that makes it potentially commercial is the development of the Co-MoCat process by Resasco's group at the University of Oklahoma, who used Co-Mo bimetallic catalysts and a fluidized-bed CVD reactor to produce a large quantity of SWNTs [14].

A recent addition to the family of CVD methods for SWNT production is the alcohol CVD method [15]. Maruyama and coworkers recently reported the synthesis of high-purity SWNTs using alcohols such as methanol and 
ethanol as a carbon source. TEM and SEM showed that the products are very clean SWNTs without any amorphous carbon coating. It is hypothesized that the $\mathrm{OH}$ radical formed at high temperature from alcohols can remove the amorphous carbon efficiently during nanotube growth, leaving only pure SWNTs as a product.

Plasma-enhanced CVD (PECVD) methods have also been widely used for making carbon materials including MWNTs. PECVD for high-quality SWNT synthesis is only recent and has been reported by several groups [16-20]. The reactive species in the plasma system could affect the growth of very small diameter tubes, with implications to both diameter control and selective etching of metallic SWNTs (Sect. 4.3). PECVD growth of large multiwall structures has also been studied extensively and has been discussed in review articles [21].

\subsection{Mass Production}

Mass production of CNTs is the key factor to realize a viable CNT industry. Among the various approaches to produce CNTs, including laser ablation [3] and arc discharge [22], CVD [4] possesses promising characteristics, such as scalability and controllability and thus is regarded as the most promising approach for mass production. Table 1 compares the relative advantages of some of the CVD methods for mass production. For MWNTs, industrial scale and economical mass production has been commercially realized by a floatingcatalyst CVD method. However, low-cost mass production of high-quality and high-purity SWNTs still remains a big challenge. Some approaches based on floating-catalyst [23] or catalyst-supported CVD methods have launched the market, but generally the cost is too high and the purity is not yet sufficient. The difficulty for mass production of SWNTs stems from the fact that a single MWNT is thousands of times heavier than a single SWNT, and thus to make the same amount of SWNTs, thousands of times more SWNTs in number have to be synthesized. In the floating-catalyst CVD method, catalytic metal nanoparticles are injected into a vertical furnace with a carbon source and SWNTs precipitate from the nanoparticles. Floating-catalyst CVD is an ideal system for MWNT growth because 1. CNTs can grow laterally by deposition of carbon on the sidewalls, and 2. catalyst particles are allowed to aggregate, enabling high-density catalyst injection into the furnace. Thus, the furnace is scalable in three dimensions. These features make the floatingcatalyst CVD method highly productive for MWNT synthesis. On the other hand, the situation is very different for SWNT growth because neither lateral growth nor catalyst aggregation are acceptable. Also, the catalyst size has to be small, in the range of a few nanometers. As such, the general strategy to synthesize SWNTs is to dilute the catalyst density in order to avoid aggregation and to shorten the growth time in order to avoid lateral growth. Therefore, scalability has a tendency to become two-dimensional, and 
Table 1. Comparison of different CVD methods for mass production

\begin{tabular}{|c|c|c|c|c|c|c|}
\hline CVD method & Temperature & Scalability & Efficiency & Purity & Quality & Alignment \\
\hline Floating catalyst & High & $\circ(3 \mathrm{D} \rightarrow 2 \mathrm{D})$ & $\circ$ & ० & ○ & - \\
\hline Fluidized bed* & Medium & ○) $(3 \mathrm{D})$ & $\circ$ & $x$ & $\circ$ & $x$ \\
\hline Supergrowth & Medium & $-(2 \mathrm{D})$ & (อ) & ○) & ० & ○) \\
\hline
\end{tabular}

Notes: *Rotary kiln with supported catalyst. Quality: ๑ Excellent.

○ Good. $\times$ Bad. - Unknown or depends.

thus the productivity of SWNTs is diminished significantly when compared to MWNT synthesis.

Another well-pursued approach is to synthesize SWNTs on supported catalysts in systems such as a fluidized bed or a rotary kiln. Generally, these approaches have high scalability. For example, metal furnaces can be used since the growth temperature is typically below $900{ }^{\circ} \mathrm{C}$, while most furnaces for floating-catalyst CVD growth are made of quartz or a ceramic because the growth temperature is usually above $1000^{\circ} \mathrm{C}$. The weak point of supportedcatalyst CVD is the low purity of the growth product, because the support material intrinsically remains as an impurity. At this moment, there seems to be no promising approach to address this key issue. Another emerging approach is to grow massive vertically aligned SWNT forests on substrates. For example, water-assisted CVD (denoted by "supergrowth", Sect. 6.1) has a very high growth efficiency $(10 \mathrm{~min}$ growth on a $29.7 \mathrm{~cm} \times 21 \mathrm{~cm}$ metal foil produces more than $1 \mathrm{~g}$ ) and can provide the purest SWNT material ever made. However, at this moment it is not obvious if this process is scalable or not. Several key points, such as large area and continuous CVD growth, need to be addressed, not to mention the issue of reducing the cost of the substrate. Finally, for SWNTs to become a widely used industrial material, eventually the cost must be reduced to the level of classic carbons, such as activated carbon or carbon fibers. This means a cost reduction of factors of hundreds to thousands must be realized in the future. All in all, so far, every existing synthetic approach has its specific weak points that need to be solved to realize mass production of high-quality, high-purity and cheap SWNTs. Only time will show which way will be the best.

\subsection{Toward Selective Synthesis}

Controlling SWNT growth by selectively forming nanotubes with desirable $(n, m)$ indices is important to obtain pure metallic or semiconducting materials needed for electronics applications, such as interconnects or transistors. Unfortunately, most of the synthesis methods produce mixtures of SWNTs with random $(n, m)$. In a few rare cases, selective synthesis phenomena have been observed. $L i$ et al. [16] reported that a low-temperature PECVD growth method selectively grew $\sim 85-90 \%$ of semiconducting SWNTs, as opposed 
to $\sim 67 \%$ of semiconductors expected for random $(n, m)$ distribution. The selective growth was attributed to the relative low heat of formation energy of semiconducting vs. metallic nanotubes, especially for SWNTs in the very small diameter range [24]. Later, Zhang et al. [25] reported selective plasma etching of metallic SWNTs over semiconducting ones, raising the possibility of plasma playing a role in selective formation of semiconducting nanotubes.

Resasco and coworkers reported SWMT synthesis with highly enriched $(6,5)$ species using a CoMo binary catalyst and CO feedstock [26]. Recently, they suggested that the $(n, m)$ distribution of the SWNT product can be reproducibly altered by varying the reaction temperature, the gaseous feed, or the cluster surface morphology [27]. The main effect of increasing the reaction temperature is to increase the metal-particle size during the SWNT growth, and consequently the nanotube diameter. By varying the support one affects the resulting morphology of the metal cluster, and as a result, the chiral angle of the nanotube produced. The interaction of the metal and the support plays an important role in the size and morphology of the cluster. It may be possible that by the appropriate selection of the growth parameters (support, gas, temperature, etc.) one can have a relatively narrow control of the $(n, m)$ distribution [27]. However, much work remains to be done to selectively grow SWNTs with specific $(n, m)$ over a wide range of diameters and chiralities.

\section{Purification}

"Purification" of carbon nanotubes is a process that separates nanotubes from non-nanotube impurities included in the raw products, or from nanotubes with undesired numbers of walls. These impurities generally include amorphous carbon, catalysts, catalyst supports, carbon nanoparticles, and unwanted nanotubes such as a small amount of MWNTs in a raw SWNT sample, or SWNTs in DWNT samples. Generally, the purification methods can be separated into dry methods and wet methods. However, the separation method varies dramatically for nanotubes produced using different methods described above, since the type of impurities included in the raw samples are quite different depending on the production methods.

Purification has been an important synthetic effort since the discovery of carbon nanotubes, and there are hundreds, if not thousands, of publications discussing different aspects of the purification process. Here, we just intend to give a brief overview of the principles with a few examples. Good review articles on the purification of nanotubes are available in the recent literature [28, 29].

\subsection{Dry Methods}

The dry methods of purification refer to methods that can selectively remove, through gas-phase oxidation, amorphous-carbon species due to their 
higher reactivity compared to that of carbon nanotubes. The simplest method is air oxidation at selected temperature, originally developed for the purification of MWNTs produced by either CVD or arc-discharge methods [30]. For SWNTs, due to their higher reactivity than MWNTs, the reaction conditions need to be adjusted from simple air oxidation to improve the selectivity of oxidation and to improve the purification yield. For example, Zimmerman et al. [31] reported a gas-phase purification method incorporating $\mathrm{Cl}_{2}$, $\mathrm{H}_{2} \mathrm{O}$, and $\mathrm{HCl}$ gaseous mixtures for SWNTs grown from pulsed-laser vaporization. SWNTs grown from the arc discharge method can be purified by oxidizing in air at $350{ }^{\circ} \mathrm{C}$ or $470{ }^{\circ} \mathrm{C}$, as described in different reports $[32,33]$. For CVD and HiPCO nanotubes, there are also many reports on their purification. For example, HiPCO nanotubes can be purified using wet air (or wet Ar- $\mathrm{O}_{2}$ ) oxidation, at $180-300{ }^{\circ} \mathrm{C}$ [34]. A scalable method to effectively purify HiPCO nanotubes was also developed by Smalley's group at Rice University by adding a step to convert metal oxide in raw samples after mild oxidation to $\mathrm{C}_{2} \mathrm{H}_{2} \mathrm{~F}_{4}$ or $\mathrm{SF}_{6}$ to form a metal fluoride that can be easily removed by $\mathrm{HCl}$ treatment [35]. Many more reports on gas-phase purification are available in the literature $[28,29]$.

\subsection{Wet Methods}

The wet methods treat nanotubes in solution for purification purposes, and can be used alone or together with dry methods. Actually, most dry methods of purification are also followed by a step of acid treatment to dissolve metal catalyst and/or metal oxides formed during the gas-phase oxidation step. Use of nitric acid $\left(\mathrm{HNO}_{3}\right)$ is the most common wet method for purification, as it is straightforward, inexpensive, and effective in removing metal catalysts and amorphous carbon from large quantities of raw material. For example, as-prepared SWNTs grown by laser-ablation techniques are known to have long bundles of SWNTs with few defects. They can be purified using nitric acid treatment [36-43]. In a typical run [41,43], raw SWNTs are refluxed in $2.6 \mathrm{M} \mathrm{HNO}_{3}$ for $45 \mathrm{~h}$. After repeated filtration steps and maybe also centrifugation steps, highly purified nanotubes can be obtained. This method is also widely adapted for the purification of nanotubes prepared by other methods with a small variation in acid concentrations. Wet purification methods can also be combined with dry oxidation methods. For example, Dillon et al. [37] have reported a nondestructive, scalable, three-step purification process that yields materials with $98 \%$ purity. Their method contains a dilute nitric acid $\left(3 \mathrm{M} \mathrm{HNO}_{3}\right)$ reflux for $16 \mathrm{~h}$, functionalized, and redistributes the nonnanotube fractions, so as to form a uniform and reactive coating on the SWNTs. This coating is selectively removed by oxidation in stagnant air. In addition, they investigated the effect of this procedure on arc discharge SWNTs, and found that a relatively long-period exposure consumed a significant fraction of the nanotubes themselves, due to the presence of a larger metal content. 
Additionally, other methods, including different chromatography methods [44-46] and centrifugation methods [47, 48], were also demonstrated to be very useful for the purification of carbon nanotubes. For example, Haddon's group at UC Riverside discovered that ultracentrifugation at different speeds can be a very effective method for the purification of SWNTs made by the arc method [47]. The method is capable of large scaleup at low cost.

\section{Sorting}

Although there has been promising progress toward the selective synthesis of carbon nanotubes of specific structure or electronic type (Sect. 2.4), all synthesis methods reported to date produce mixtures of carbon nanotubes of different structures and types. Therefore, significant research is currently being devoted to developing methods for sorting these mixtures into specific structures or types $[29,49]$. By "sorting", one ideally refers to the partition of a mixture into physically separated ensembles of carbon nanotubes of a particular type (metallic or semiconducting) or structure (length, diameter, chirality), or, even more ideally, a specific pair of $(n, m)$ indices. Less ideally, but often more realistically, one can also refer to partial sorting or "enrichment" of samples having a higher proportion of a particular type, or "fractionation" into ensembles having a narrower distribution of diameters or lengths.

During the last five years, significant progress in carbon nanotube sorting has been enabled by the development of new methods of dispersing carbon nanotubes in solution [50], as well as by new methods of carbon nanotube metrology (see contribution by Jorio et al.), which are critical for determining the distribution of structures in carbon nanotube samples. The dispersion of discrete carbon nanotubes in solution and their systematic population analysis have also enabled the study of selective interactions between external forces or chemical species and carbon nanotubes of different structures and/or types. Any of these selective interactions can in principle be exploited for the sorting of carbon nanotubes. This section reviews the different methods of carbon nanotube sorting, and also the different selective interactions, which could in principle become the basis for future sorting methods. Rather than sequentially reviewing the more than 70 reports related to the topic of carbon nanotube sorting, we shall first attempt to classify them in a rational way by their operating principles. We then describe some of the most promising methods of sorting reported so far, and finally try to draw some conclusions and perspectives toward the ideal sorting of carbon nanotubes.

\subsection{Classification of Sorting Methods and Selective Processes}

The different methods of carbon nanotube sorting, and selective interactions or processes, which could eventually lead to new methods of sorting, may be rationally classified by the following aspects: 
1. Parameter of selectivity (from more general to more specific): length $(L)$, electronic type $(\mathrm{M} / \mathrm{S})$, diameter $\left(d_{\mathrm{t}}\right)$, chirality $(\theta)$, and structure $(n, m)$.

2. Type of selective force or interaction (from weaker to stronger, or from longer to shorter range): gravity (e.g., in sedimentation), inertial forces (e.g., in centrifugation), volume-exclusion forces (e.g., in size-exclusion chromatography), electric-field (e.g., dielectrophoresis), selective noncovalent adsorption (e.g., by van der Waals forces, charge-transfer, $\pi$-stacking, etc.), reversible covalent functionalization, irreversible chemical reaction, and finally, a selective elimination by a chemical (e.g., etching) or physical (e.g., electrical breakdown) process.

3. Type of medium (from less to more condensed): vacuum, plasma, gas, organic solvent, aqueous solution, gel, and solid.

4. Degree of separation (from lower to higher): selective orientation (e.g., by electric field), selective modification (e.g., adsorption, reaction, etc.), microscopic selection (e.g., selective deposition of individual nanotubes), bulk enrichment, and finally, complete bulk separation.

5. Production scale or scalability, namely, what amount of nanotubes are sorted, or can be sorted, using a particular method.

Combinations of different selection parameters, types of interactions, or types of medium are also possible.

Table 2 summarizes different reported methods of sorting and selective processes, with their respective references, classified by the parameter of selectivity and the type of selective force or interaction. Specific subgroups of interactions are listed in italics. The type of medium is indicated in parentheses, unless it is an aqueous dispersion with common ionic or nonionic surfactants, such as sodium dodecylsulfate (SDS), sodium dodecylbenzenesulfonate (SDBS) or Triton X-100, assuming that the interaction with the surfactants is not the main selective agent. In each field, the different items are listed by increasing degree of separation (4). Items in black indicate actual sorting or enrichment methods, which are in principle scaleable, whereas items in gray are selective processes not attaining a physical separation of the different types of nanotubes. Specific remarks are listed below the table.

\subsection{Nondestructive Sorting}

Four exemplary nondestructive methods of carbon nanotube sorting selected from Table 2 are schematically represented in Fig. 1, and explained below (other methods have been described in recent reviews [29, 49]).

\section{Dielectrophoresis (Fig. 1a)}

This was the first demonstrated method of sorting by electronic type [59]. In this method, a surfactant-stabilized aqueous suspension of single-wall carbon nanotubes is placed onto an array of interdigitated electrodes, across 
Table 2. Classification of sorting methods and selective processes

\begin{tabular}{|c|c|c|c|}
\hline & $L$ & $\mathrm{M} / \mathrm{S}$ & $d_{\mathrm{t}}$ \\
\hline Gravity & & Precipitation $^{1}$ & \\
\hline Inertial forces & & Centrifugation $^{1}$ & \\
\hline $\begin{array}{l}\text { Hydrodynamic } \\
\text { forces }\end{array}$ & $\begin{array}{l}\text { Field-flow } \\
\text { fractionation }[43,51]\end{array}$ & & \\
\hline $\begin{array}{l}\text { Volume- } \\
\text { exclusion } \\
\text { forces }\end{array}$ & $\begin{array}{l}\text { Size-exclusion } \\
\text { chromatography [44, } \\
45,52-56]\end{array}$ & Filtration $^{1}$ & \\
\hline Electric field & $\begin{array}{l}\text { Electrophoresis [57, } \\
58]\end{array}$ & $\begin{array}{l}\text { Dielectrophore- } \\
\text { sis [59-67] }\end{array}$ & Electrophoresis [58] \\
\hline $\begin{array}{l}\text { Noncovalent } \\
\text { adsorption }\end{array}$ & & $\begin{array}{l}\mathrm{RNH}_{2}(\mathrm{THF})^{2}+ \\
\text { filtration }[68,69] ;+ \\
\text { centrifugation }[70,71]\end{array}$ & \\
\hline Van der Waals & $\begin{array}{l}\text { Extraction TOAB } \\
\left(\mathrm{H}_{2} \mathrm{O} / \mathrm{EA}\right)^{3}[72]\end{array}$ & & $\begin{array}{l}\text { Encapsulation of } \\
\text { metallocenes [73] } \\
\text { Reversible cyclic } \\
\text { peptides }+ \\
\text { centrifugation [74] }\end{array}$ \\
\hline Charge transfer & & $\begin{array}{l}\mathrm{Br}_{2}+ \\
\text { centrifugation [75] }\end{array}$ & $\begin{array}{l}\text { Electrochemical } \\
\text { doping }[76] \\
\mathrm{FeCl}_{3}[77,78] \\
\mathrm{K}[77,78]\end{array}$ \\
\hline$\pi$ stacking & & $\begin{array}{l}\text { Porphyrins }+ \\
\text { centrifugation [79] }\end{array}$ & $\begin{array}{l}\mathrm{DNA}+ \\
\text { centrifugation [80] }\end{array}$ \\
\hline $\begin{array}{l}\text { Reversible } \\
\text { covalent func- } \\
\text { tionalization }\end{array}$ & & $\begin{array}{l}\mathrm{RN}_{2}^{+}+\mathrm{e}^{-}[66] \\
\mathrm{RN}\left(\mathrm{CH}_{2}\right)_{2}^{-}+ \\
\text {precipitation [81] }\end{array}$ & \\
\hline $\begin{array}{l}\text { Chemical } \\
\text { reaction }\end{array}$ & & $\mathrm{OsO}_{4}+h \nu \quad[82]$ & \\
\hline $\begin{array}{l}\text { Chemical } \\
\text { decomposition }\end{array}$ & & $\begin{array}{l}\mathrm{RN}_{2}^{+}(\mathrm{l} / \mathrm{s})[83] \\
\mathrm{H}_{2} \mathrm{O}_{2}[84] \\
\mathrm{H} \text { plasma }[85] \\
\mathrm{CH}_{4} \text { plasma }(\mathrm{g})[86]\end{array}$ & $\begin{array}{l}\mathrm{HNO}_{3}[87] \\
\mathrm{O}_{2}(\mathrm{~g})[87] \\
\mathrm{H}_{2} \mathrm{SO}_{4} / \mathrm{H}_{2} \mathrm{O}_{2}[88]\end{array}$ \\
\hline $\begin{array}{l}\text { Physical } \\
\text { destruction }\end{array}$ & & $\begin{array}{l}\text { Electrical breakdown } \\
(\mathrm{g} / \mathrm{s})[89,90]\end{array}$ & \\
\hline
\end{tabular}

Notes: ${ }^{1}$ These processes are not selective per se, but are used for physical separation in combination with the selective processes listed below. ${ }^{2} \mathrm{THF}=$ tetrahydrofurane. ${ }^{3} \mathrm{TOAB}=$ tetraoctylammonium bromide, $\mathrm{EA}=$ ethyl acetate 
Table 2. continued

$\mathrm{M} / \mathrm{S}+d_{\mathrm{t}} \quad \theta \quad n, m$

Gravity

Inertial forces

Hydrodynamic

forces

Volume-

exclusion

forces

Electric field Dielectrophoresis

field-flow

fractionation [91]

Noncovalent $\quad \mathrm{RNH}_{2}(\mathrm{THF})^{2}+$

adsorption filtration [92]

Van der Waals

Bile salts +

density-gradient

centrifugation [93]

Charge transfer $\mathrm{Fe}(\mathrm{CN})_{6}^{3-}[94]$

$\pi$ stacking

$\mathrm{DNA}+$ ion-exchange chromatography [95-98]

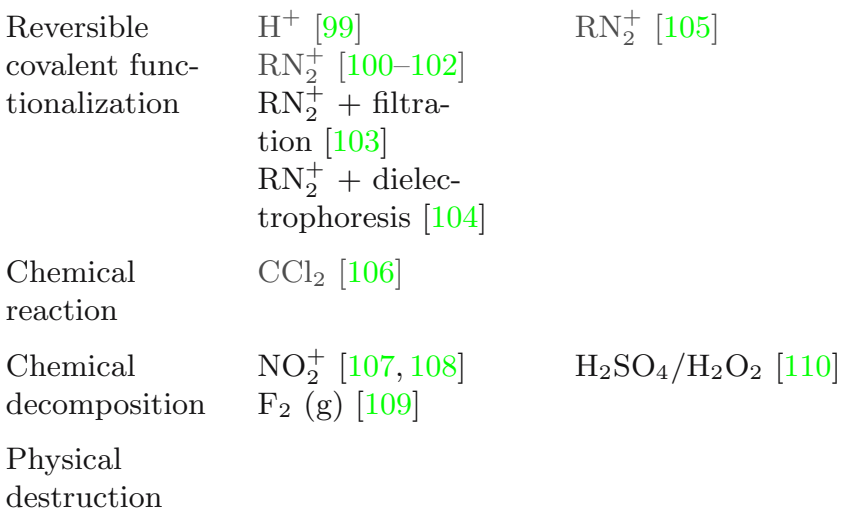

Notes: ${ }^{1}$ These processes are not selective per se, but are used for physical separation in combination with the selective processes listed below. ${ }^{2} \mathrm{THF}=$ tetrahydrofurane. ${ }^{3}$ TOAB $=$ tetraoctylammonium bromide, $\mathrm{EA}=$ ethyl acetate 
which an ac voltage of $10 \mathrm{~V}$ at $10 \mathrm{MHz}$ is applied for a few minutes. The dielectrophoretic force acting on a particle is proportional to the field square gradient and the Clausius-Mossotti factor $\left(\varepsilon_{\mathrm{p}}-\varepsilon_{\mathrm{m}}\right) /\left(\varepsilon_{\mathrm{p}}+2 \varepsilon_{\mathrm{m}}\right)$, where $\varepsilon_{\mathrm{p}}$ and $\varepsilon_{\mathrm{m}}$ are the dielectric constants of the particles and the medium, respectively. The dielectric constant of metallic SWNTs is larger than that of water, whereas that of semiconducting nanotubes is actually smaller, so the Clausius-Mossotti factor is positive for metallic nanotubes but negative for semiconducting ones. Hence, only the metallic carbon nanotubes are attracted toward the electrodes, and selectively deposited onto them, while the semiconducting nanotubes remain in the suspension. This method can yield $\sim 100 \mathrm{pg}$ quantities of carbon nanotubes enriched with $80 \%$ metallic nanotubes. Interestingly, the Clausius-Mossotti factor can be modified by varying the frequency [61] or by chemical functionalization [104], thus allowing different sorting possibilities. The method can be used for depositing thin films of aligned metallic nanotubes [67], and can in principle be scaled up using macroscopic electrodes [64], filtration [62], and microfluidics. Dielectrophoretic field-flow fractionation is an interesting way of combining the advantages of dielectrophoresis and field-flow fractionation [91], which also allows sorting by diameter. In any case, the fact that dielectrophoresis requires an inhomogeneous field means that its scaling up for mass production may not be trivial.

\section{Covalent Functionalization (Fig. 1b)}

This is a more classically chemical approach to type and diameter sorting. Diazonium ions $\left(\mathrm{RN}_{2}^{+}\right.$, where $\mathrm{R}$ is an aromatic group, such as 4-chlorophenyl) were shown to react selectively with metallic carbon nanotubes of small diameters [100]. Similar selective functionalization with diazonium ions having the bulky group $\mathrm{R}=4$ tert-butylphenyl enabled subsequent separation by filtration on silica gel [103]. After separation, the metal-enriched functionalized SWNTs could be defunctionalized into pristine SWNTs by thermal treatment. This process can in principle be performed in a chemical reactor, so it could be massively scaled up.

\section{DNA-Assisted Dispersion (Fig. 1c)}

This is an interesting pathway for type and diameter sorting [95]. Singlestranded DNA was shown to wrap around SWNTs, allowing their dispersion in aqueous solutions. The DNA-wrapped SWNTs are negatively charged, and can then be fractionated by ion-exchange chromatography, yielding a fraction enriched in metallic or semiconducting SWNTs with different diameter ranges. The use of a specific DNA sequence consisting of a d $(\mathrm{GT})_{10-45}$ repeat (i.e., 10 to 45 alternate $G$ and $T$ bases) significantly enhances the structure-based sorting of SWNTs, producing highly enriched fractions of 

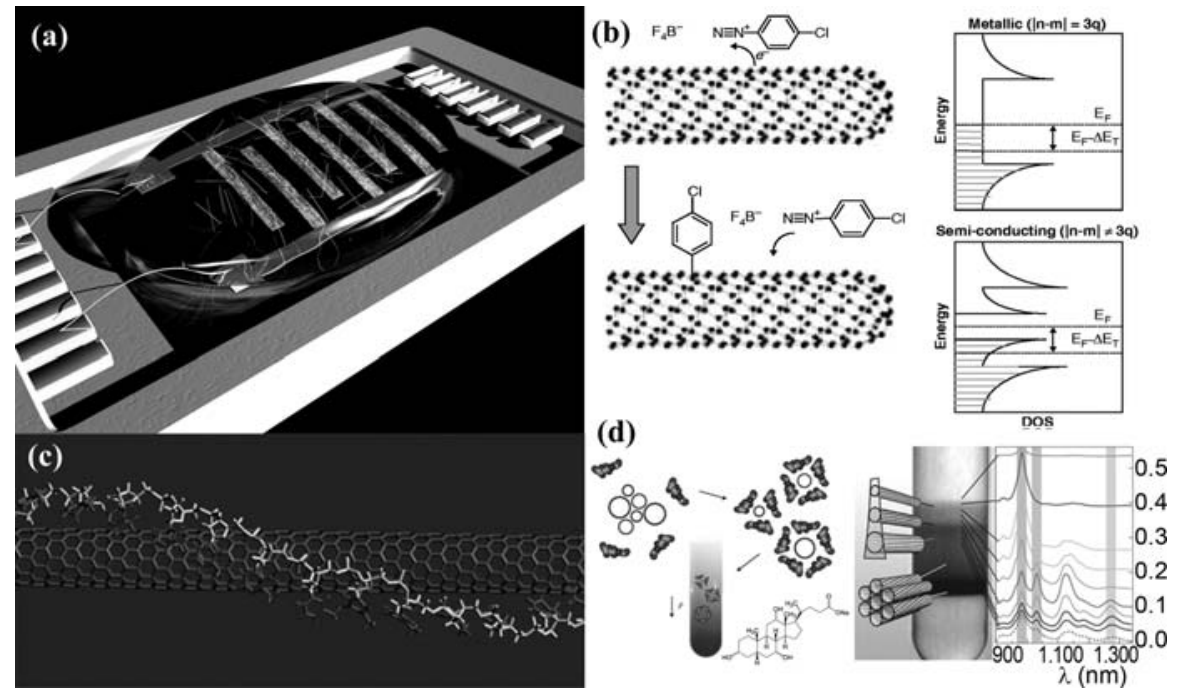

Fig. 1. Exemplary nondestructive methods of carbon nanotube sorting. (a) Dielectrophoresis [59]. (b) Covalent functionalization [100] $\left(E_{\mathrm{F}}\right.$ : Fermi energy; $\Delta E_{\mathrm{T}}$ : charge-transfer energy). (c) Ion-exchange chromatography with DNA. Model of a DNA-wrapped SWNT [95]. (d) Density-gradient centrifugation with bile salts (e.g., sodium cholate) [93]

specific $(n, m)$ [96]. This process is scalable in principle, but relatively expensive due to the high price of oligonucleotides.

\section{Density Differentiation with Bile Salts (Fig. 1d)}

This is a very promising recent approach for $(n, m)$ sorting [93]. Bile salts are relatively bulky and rigid surfactants that are naturally derived from cholesterol. They encapsulate SWNTs in such an arrangement that the density of the supramolecular assembly depends on the nanotube diameter. Hence, ultracentrifugation in a density gradient leads to separation by type and diameter. Using narrowly dispersed starting materials, such as CoMoCATgrown SWNTs, and repeating the separation with the right combination of surfactants, allow the production of highly enriched fractions with single $(n, m)$ structures. The fact that bile salts are abundant byproducts of the meat-processing industry, and the relative simplicity of the centrifugation technique, could make this sorting method highly scalable and inexpensive.

\subsection{Selective Elimination}

The last two rows of Table 2 show different sorting methods based on selective elimination. Such methods may be especially important for the elimina- 
tion of metallic nanotubes from nanotube-based electronic circuits in situ, in order have only semiconducting nanotubes to operate as field-effect transistors (FETs). Selective physical destruction of metallic nanotubes by electrical breakdown has been used for this purpose [89, 90]. However, this method requires the nanotubes to be connected to electrodes, which is a limitation for large-scale integration of nanocircuits. Dai and coworkers [25] have recently developed a process of methane plasma followed by annealing, to selectively etch or hydrocarbonate M-SWNTs and retain S-SWNTs in the 1-2 nm diameter window. It is revealed that diameter is an important factor in the chemical reactivity of a SWNT towards hydrocarbonation and etching. Smallerdiameter SWNTs (below $\sim 1.2 \mathrm{~nm}$ ) are preferentially etched over larger ones due to the higher radius of curvature and higher strain in the $\mathrm{C}-\mathrm{C}$ bonding configuration. In a certain diameter range, metallic tubes are selectively etched over semiconducting ones due to more abundant delocalized electronic states. The difference in chemical reactivity diminishes for SWNTs with large diameters ( $\sim 2 \mathrm{~nm}$ and larger $)$ without selectivity [25].

The gas-phase etching method is reliable and can provide $100 \%$ yield of semiconductors in an ensemble of SWNTs. The retained S-SWNTs are free of covalent alterations upon thermal treatment and thus exhibit electrical properties similar to pristine materials. The distribution of diameters of the S-SWNTs is narrowed down to a window $(\sim 1.3-1.6 \mathrm{~nm})$ that provides sufficient bandgaps for high on/off ratios and allows for good electrical contacts, both of which are important for high-performance electronics [25]. The dual effects of selective metal removal and diameter-distribution narrowing combined with compatibility with microfabrication technology make the method promising for large-scale SWNT electronics. This method was used to demonstrate FETs with large numbers of S-SWNTs in parallel by selectively etching metallic SWNTs in a large ensemble of tubes. About 20 intact semiconducting SWNTs in parallel in SWNT devices were obtained without any metallic short [25].

\subsection{General Principles and Perspectives of Sorting}

By looking at Table 2 and considering the selected examples, one can learn several interesting principles: Length-sorting methods are usually based on relatively weak and long-range forces, such as in field-flow fractionation $[43,51]$, size-exclusion chromatography [44, 45, 52-56], and electrophoresis $[57,58]$. One interesting exception is the length-dependent extraction in a two-phase liquid-liquid system using a phase-transfer chemical agent [72]. In contrast, methods for sorting by type and diameter (and combinations thereof) are usually based on stronger or shorter-range forces, from electric fields to covalent bonding. Nondestructive selective interactions with chemical species cannot by themselves lead to sorting, unless they are combined with an additional weaker or longer-range force, which is used to physically 
separate the different nanotubes, for example by precipitation [81], centrifugation [70, 71, 74, 75, 79, 80, 93], filtration [68, 69, 92, 103] or chromatography [95-98]. In these cases, the selective interaction with chemical species will lead to a physical separation based on chemically induced differences in solubility [68,69,74,81,92,103], density [75,93] or charge [95-98]. It is interesting to note that type and diameter selectivity often come together. This can often be attributed to the fact that smaller-diameter nanotubes have a larger bandgap and a higher strain. Hence, the diameter also affects the physical and chemical properties of the nanotubes. In general, metallic carbon nanotubes are more reactive than semiconducting ones toward covalent functionalization due to their higher density of states near the Fermi energy [100], resulting in stronger orbital interactions [111,112]. However, semiconducting-selective reactions have also been reported [79]. Smaller-diameter nanotubes are generally more reactive toward covalent functionalization due to their higher strain and $\mathrm{sp}^{2}$-orbital pyramidalization [113]. On the other hand, their doping or charge-transfer functionalization is more favorable for large-diameter nanotubes due to their smaller bandgap [76,77,113]. Interestingly, there are very few reports of chirality-dependent processes [105, 110], and their chiral dependence can actually be attributed to a combination of type and diameter selectivity. The lack of intrinsically chirality-dependent processes may indicate that chirality per se does not strongly affect the chemical or physical properties of the nanotubes, but rather their type and diameter. Moreover, there is currently no method for chirally resolving left-handed from right-handed nanotubes. One could envisage a chiral-dependent process by a commensurate interaction between the rolled graphene sheet and a chiral crystalline surface or a helical overlayer of chiral molecules, but such resolution has not yet been reported. Finally, the ultimate sorting of unique $(n, m)$ structures has so far been achieved by highly type- and diameter-selective processes using narrowly distributed starting materials [93, 95]. From these principles, it may be concluded that the most promising pathway for the production of pure $(n, m)$ samples could be a skillful combination of a selective synthetic method (Sect. 2.4) with a highly type- and diameter-selective sorting method, based in turn on a combination of chemical modification with an effective means of physical separation. Amplification of $(n, m)$-sorted SWNT "seeds" by continued growth [114-116] could make the sorting process even more scalable in an analogous way as the synthesis of DNA has been boosted by the invention of a polymerase chain reaction (PCR). If progress continues at a good pace, the day when one can order carbon nanotubes with specific $(n, m)$ and length from a catalog may not be too far away.

\section{Organization into Fibers}

Carbon nanotubes, both single wall and multiwall have beguiling axial properties. Mechanically, they have been shown to be very strong and stiff, elec- 
trically they are conducting, and in the case of single-wall tubes, the nature of the conductivity, ranging from metallic to a large-band-gap semiconductivity, depends critically on how the graphene sheet is joined up to make the tube, in fact on its chirality. They also show promise as thermal conductors.

The challenge is to organize these individual nanotubes into a material that will transfer their individual properties into an exploitable material form. In the case of the axial properties of carbon nanotubes, the obvious material form is a fiber in which the nanotubes are well aligned with the axis and as closely packed as possible. There is a broad spectrum of research aimed at maximizing the property transfer from the nano- to the macroscale, and it reflects one of the central themes of materials science, namely the organization of atomic, molecular, nano- and microscales of structure to realize desired properties at the macroscale. The properties of the individual nanotubes, the building blocks for the fiber, are crucial in determining the final properties, for it is all but impossible that these will be enhanced on fiber forming, and in general they will be degraded.

This section focuses on the developing technologies that are providing routes to the production of fibers consisting of carbon nanotubes. However, there are many options for incorporating varying amounts of a second material, typically a polymer, within the fiber. Of course there is a continuous range of compositions here, ranging from pure nanotube fibers, through property-modified nanotube fibers, through composites, through to what are really no more than nanotube-filled polymer fibers, and finally through to pure polymer fibers themselves. An arbitrary limit is set in this section, in that only fibers with concentrations greater than $20 \%$ carbon nanotubes are discussed, and where the nanotubes are the minority component, then the descriptions are limited to processes that can also make quite pure nanotube fibers. Hence, coagulation spinning of composite fibers is included, but not melt extrusion and drawing of a polymer containing carbon nanotubes as a filler, as in this latter case the enhancement of the melt viscosity by the nanotubes makes such processing difficult above concentrations of $10-20 \%$. There is also another focus, and most attention is given to processes that have the ability to spin fibers continuously.

\subsection{Processing Principles}

The task is clear, we have to start with carbon nanotubes in some form, and organize these into a fiber, while at the same time, as far as is possible, align them perfectly with the fiber axis. Carbon nanotubes are long and thin, and in the case of single-wall tubes, their diameter is similar to that of many polymer molecules (e.g., the PMMA molecule is $\sim 0.7 \mathrm{~nm}$ in diameter). They are, however, very stiff compared with even the stiffest polymer molecules. Computational modeling [117] indicates that the persistence length of even a small-diameter single-wall carbon nanotube $(7,7)$ is some tens of micrometers, which would make it some 100 times stiffer than DNA. While such a 
value may be surprising, it should be remembered that a thin-walled tube is amongst the stiffest, per unit mass, of all engineering structures, and in the case of carbon nanotubes bending requires the stretching and compression of the strong carbon-carbon bonds. A direct consequence of this stiffness is that assemblies of carbon nanotubes are not expected to melt to form any freeflowing phase. The melting point of any crystal is given by $T_{\mathrm{m}}=\Delta H / \Delta S$. In the case of nanotubes, the $\Delta S$ term is vanishingly small on two counts. Firstly, their intrinsic stiffness means they are all but as rigid in the disorganized state than when packed parallel on an hexagonal net; secondly they are large compared with individual atoms so the entropy of mixing is correspondingly very small. The melting point is thus effectively "off scale", and melt-processing routes are not available. It is therefore necessary to look at other options. There are two: creating free-flowing suspensions in a low molecular weight liquid or applying mechanical fields to dilute assemblies of nanotubes in a gas phase. In each gas the dilution of the nanotubes will enable them to be oriented by the field as it forms the fiber. These two approaches, which may be classified as "wet" and "dry", form the basis of all the successful process strategies for the formation of carbon nanotube fibers. A final method, which is currently being reduced to practice, involves the extension of an existing fiber by continual synthesis and deposition of carbon nanotubes onto its end [115]. In the descriptions below, the wet routes will be explored first, and then the dry.

\subsection{Liquid Suspensions of Carbon Nanotubes}

With so little entropy to be gained through distributing nanotubes in a liquid medium, it has to be achieved by treating the system so that there is a clearly negative heat of mixing $\left(\Delta H_{\text {mix }}\right)$, or in other words, there needs to be a preference for bonds to be formed between the nanotube surface and the molecules of the liquid. Such a favorable heat of mixing can be achieved either by a very careful choice of solvent [118], or by surface treating the carbon nanotubes so that they are compatible with the suspending liquid [119] or through the use of surfactants, i.e., small molecules that have a liquid compatible part and a carbon nanotube-compatible part [52]. These strategies continue to evolve. A key aspect of liquid suspensions of long rigid rods is that they can organize in two ways, they can assume random orientations (bag of nails) but with very inefficient packing so that such structures will be associated with high dilutions. Alternatively, the rods can pack parallel, but with solvent molecules still between them. Such a phase is a solvent stabilized, or, specifically a lyotropic, liquid-crystalline phase. The relationship between the concentration of rigid rods in a suspending medium, and either the heat of mixing $\left(\Delta H_{\text {mix }}\right)$, or the temperature, is shown as a schematic phase diagram in Fig. 2, first predicted by Flory [120]. Song et al. [121], first demonstrated nanotube liquid crystallinity using aqueous suspensions of multiwall nanotubes. They extended the study to topological defects in the 


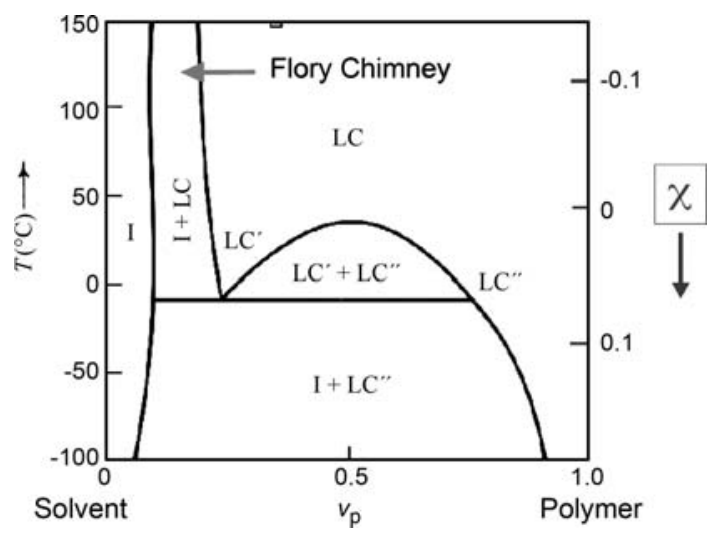

Fig. 2. Schematic phase diagram for a system consisting of a liquid containing rigid rods in suspension. It shows isotropic and liquid-crystalline phase fields (I) and (LC). The diagram does not allow for the possibility of crystallization of the rods at high concentrations. The diagram is drawn here as a function of temperature and $\chi$, which is a variable related to the heat of mixing, $\Delta H_{\text {mix }}$

director fields relating these back to the precepts of liquid-crystal science. Incidentally, where there is a distribution of lengths and perfection amongst any suspension of carbon nanotubes, it has been shown that, for concentrations within the two-phase region or "Flory chimney", the longer, straighter tubes partition into the liquid-crystalline phase, with the poorer material being rejected into the isotropic phase. Such partition has recently been exploited in a fractionation process [122], which has relevance as the longer, straighter nanotubes are known to form stronger fibers, as is discussed further below. Liquid crystallinity is an example of self-organization, and such phases are known to be an excellent precursor for obtaining high orientation in fibers spun from the liquid, the process for making aramid fibers being the most important example, e.g., [123].

\subsection{Spinning Carbon Nanotube Fibers from Liquid-Crystalline Suspensions}

The first successful spinning of a continuous single-wall carbon nanotube fiber from a liquid-crystalline suspension was reported in 2004 by Ericson et al. [124] from Rice University. The suspending medium for an $8 \%$ dispersion single-wall nanotube, which had been synthesized from carbon monoxide in the HiPCO method, was $102 \%$ sulfuric acid ( $2 \mathrm{wt} \%$ excess $\mathrm{SO}_{3}$ ). The suspension was stabilized by the formation of a charge-transfer complex as the acid protonated the nanotubes. The solution, which had to be kept completely dry, was extruded into an aqueous coagulation bath where the sulfuric acid was removed, leaving a fiber of the order of $50 \mu \mathrm{m}$ diameter. Figure 3 shows a WAXS diffraction pattern of the fiber (fiber axis vertical) 


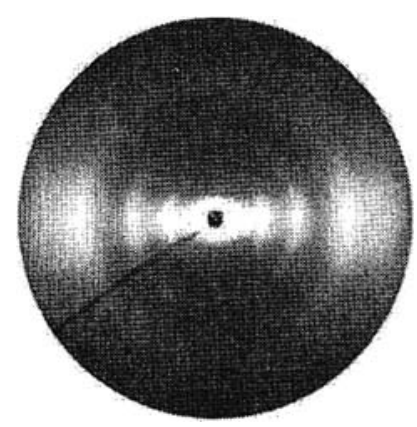

Fig. 3. Wide-angle X-ray diffraction picture of a single-wall carbon nanotube fiber drawn from a liquid-crystalline suspension in concentrated sulfuric acid. The pattern indicates high-quality alignment of the nanotubes with the fiber axis [124]

and an azimuthal scan yielded a peak width of $31^{\circ}$ (at half-maximum). The fibers showed a strength of $0.16 \mathrm{GPa}$ and a stiffness of $120 \mathrm{GPa}$.

Two other pieces of work have explored the production of fibers by essentially the same route as the Rice work, but using rather more amenable solvents. Steinmetz et al. [125] spun from a stabilized aqueous suspension of arc-grown single-wall nanotubes into a coagulation bath of either ethylene glycerol or ethylene glycol, to which 1\% SDS had been added to act as a surfactant. The fibers were then washed to remove the surfactant. By way of comparison, Zhang et al. [126] spun from an ultrasonically stabilized suspension of multiwall nanotubes in glycol into a coagulation bath of ether. The processes produced fibers of strengths of $\sim 0.1 \mathrm{GPa}$ (inferred) and $0.20 \mathrm{GPa}$, respectively. The elastic moduli, however, were $2 \mathrm{GPa}$ and $130 \mathrm{GPa}$ for the two processes. The electrical conductivities were also very different, being $700 \mathrm{~S} / \mathrm{m}$ and $8000 \mathrm{~S} / \mathrm{m}$. Zhang et al. also measured the quality of the alignment in a way that permits comparison with the sulfuric acid spinning of [124]. The Zhang value was $20^{\circ}$ width at half-height for the azimuthal peak, indicating somewhat better alignment for the multiwall nanotube specimen.

It would appear that all three of these spinning processes were from lyotropic liquid-crystalline suspensions, although Steinmetz et al. do not say so explicitly. The strengths, and in the case of the single-wall surfactant dispersed tubes, the elastic modulus, are disappointing. Various authors across the field have commented that a central factor in maximizing strength will be the lengths of the individual nanotubes $[127,128]$, in much the same way as the strength of polymeric fibers is optimized at high molecular weights. Strengths are also likely to be enhanced by crosslinks between individual nanotubes [125].

\subsection{Wet Spinning of CNT Composite Fibers}

It appears that the first successful spinning of a carbon nanotube-bearing fiber was by Vigolo, Poulin and coworkers in a sequence of papers stemming from Vigolo et al. in 2000 [129]. In [130] the team reports the spinning of continuous lengths of a composite fiber consisting of a significant concentration 


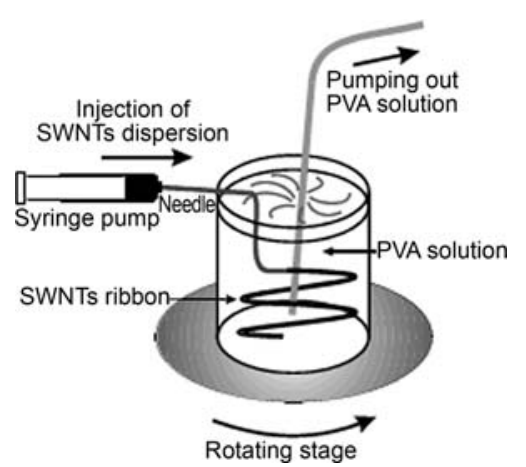

Fig. 4. The wet spinning method developed by Vigolo, Poulin and co-workers [130]. An aqueous suspension of carbon nanotubes, stabilized by a surfactant, is injected into a flowing solution of polyvinyl alcohol which causes the fiber to coagulate. It is then removed from the bath and washed to remove excess polymer. The resultant fiber is a composite, typically $50 / 50$ of carbon nanotubes and polymer

of nanotubes in polyvinyl alcohol (PVA). They set the work in the context of other alignment methods such as electrophoretic nanotube alignment for short fiber lengths [131], and also provided a good basis for the use of surfactants to achieve the dispersions. Figure 4 [130], shows the essence of the process. A dilute surfactant-stabilized aqueous suspension of single-wall carbon nanotubes, (CNT concentration $<1 \%$, surfactant, SDS, concentration $<5 \%)$ is injected into a flowing bath of PVA solution in water. The PVA replaces the water causing the fiber to coagulate. The fibers had a strength of $0.15 \mathrm{GPa}$ and a stiffness of $15 \mathrm{GPa}$. The work sparked a development process involving stretching of "rewetted" fibers [132], to give a strength of $0.2 \mathrm{GPa}$ and a stiffness of $40 \mathrm{GPa}$.

Further developments have followed by the French group [133, 134], who have shown that for SWNT PVA ratios in the region of 50/50, that in addition to improved strength and stiffness, very high toughness fibers can be obtained. In particular, the process of hot drawing of the composite [133] seems to provide an exciting enhancement of toughness at low strains, with a strength of $1.5 \mathrm{GPa}$ and a toughness (at $11 \%$ fracture strain), of $55 \mathrm{~J} / \mathrm{g}$. The orientation of the PVA molecules in such samples is of a particularly high order. Figure 5 shows the stress/strain data from [133] correlated with the WAXS patterns indicating the degree of alignment in the fibers. In a parallel development involving coaxial flow, fibers of strengths as high as 1.8 GPa [135] have been seen. Salt routes for nanotube dispersion, where a sodium salt is made in association with the nanotube that then carries charge, possibly as much as one unit per ten carbon atoms, are a further important step [136]. When placed in a polar solvent the charged nanotubes form a suspension without the need for sonication and the possible consequence of a reduction in fiber lengths. Applying this suspension route to the coagulation process [127], produced mechanical properties close to those for the SDSspun material, even with only $15 \%$ CNT loading compared with $50 \%$ for the surfactant suspensions. Wet-spun composite fibers are unlikely to be good candidates for high electrical conductivity owing to the insulating polymer separating the nanotubes, and there are correspondingly only sparse reports 


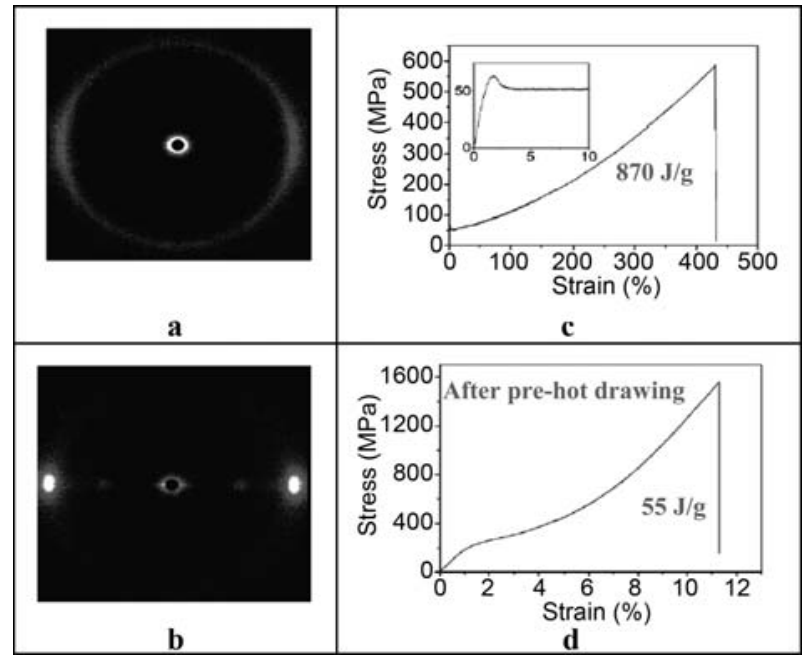

Fig. 5. More recent results from the wet-spinning method showing X-ray and stressstrain data from wet-spun composite fibers (SWNT/polyvinyl alcohol), incorporating additional alignment procedure during spinning, (a) to give a remarkable tough fiber by any comparative standard, albeit measured up to a breaking strain of $430 \%$ and (b), the result of hot drawing the fiber, giving much better orientation of both the nanotubes and the matrix polymer molecules, and a resultant increased fracture stress, whilst retaining a significant energy to break for only $11 \%$ elongation [133]

of such measurements. However, values in the range of $4-300 \mathrm{~S} / \mathrm{m}$, have been quoted for SDS wet-spun CNT fiber.

The coagulation spinning technique is versatile, and has been used by several workers to generate fibers with a wide range of polymers with comparatively low nanotube loadings for specific purposes. References [137-140], for example, cover polyanaline, polyacronitrile and UHMW polyethylene.

\subsection{Dry Spinning from Carbon Nanotube Forests}

Carbon nanotubes, especially multiwall ones, can be grown as "forests" from a flat substrate with comparative ease (Fig. 6a). The nanotubes are relatively straight and thus have only modest levels of entanglement. Jiang et al. [141] first demonstrated that for forests grown under the right conditions, the nanotubes can be drawn away from the forest edgeways to form a very thin coherent film that can then be twisted into a fiber.

A process established for continuous wind-up [142] has provided an excellent basis for determining the influence of twist on strength, where the relationships between twist angle and nanotube lengths can be analyzed using the precepts of yarn technology, as it applies to the creation of continuous yarn from twisted short fibers (staple) [143]. Figure 6b shows the process in operation. The twisted yarn in this example is in fact very fine ( $2 \mu \mathrm{m}$ diameter), 

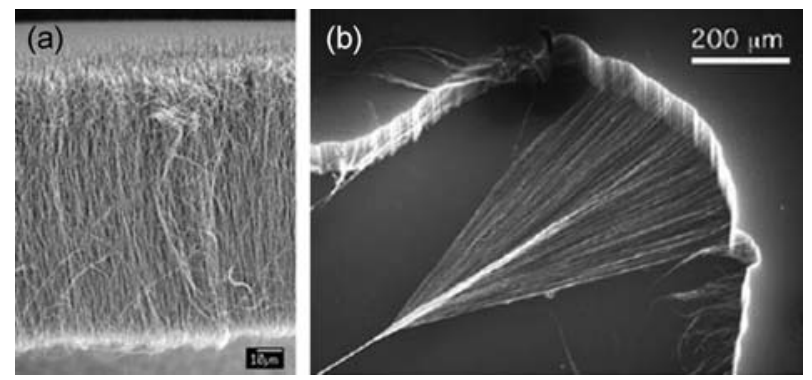

Fig. 6. Dry spinning from carbon nanotube forests. (a) A typical "forest" of multiwall carbon nanotubes grown from a substrate, in this case a silica plate. (b) Photograph of the spinning of a carbon nanotube fiber from a forest of multiwall nanotubes. The fiber is drawn at right angles to the direction of the nanotubes in the forest [142]

and could be difficult to see with the naked eye. The mechanical properties first reported were tensile strengths in the range of $0.3-0.8 \mathrm{GPa}$ and stiffnesses up to $25 \mathrm{GPa}$. The electrical conductivity reported was $30 \times 10^{3} \mathrm{~S} / \mathrm{m}$ (compared to $\mathrm{Cu}: 60 \times 10^{6} \mathrm{~S} / \mathrm{m}$ ). With the twist optimized, one route towards increased mechanical and electrical properties is likely to be the growth of forests of ever-increasing length $[128,144]$. There is also the possibility of exploiting the thin films drawn as they first emerge from the forest. They are exceptionally thin and transparent; they also show amazingly high strengths on a mass per unit area basis [145].

\subsection{Direct Spinning from Carbon Nanotube Fibers from the CVD Reaction Zone}

In all the techniques described so far, the carbon nanotubes have to be synthesized in one stage, and the fibers spun as a second distinct step. The method of spinning nanotube fibers directly from the CVD reaction zone was introduced by $L i$ and coworkers [146]. There have been numerous reports in the literature of fibrous-like deposits in CVD reaction chambers. Of these Zhu et al. [147] were the first to measure mechanical properties of $20 \mathrm{~cm}$ strands, which they found were significant with elastic moduli of the order of $50 \mathrm{GPa}$ and strengths of $1 \mathrm{GPa}$. The Cambridge process [146] took the significant step of realizing that the carbon plume formed in the reaction chamber, where the floating catalyst of iron was introduced into the furnace as ferrocene dissolved in the liquid hydrocarbon feedstock, had elastic properties owing to the entanglement of the long nanotubes. This elastic smoke can be "hooked" and wound up continually either within the furnace or outside. The geometry can be varied to form either a fiber or a film (Figs. 7 and 8). The elastic smoke is technically an aerogel. Its condensation into fiber or film geometries is either achieved through the particular wind-up geometry em- 


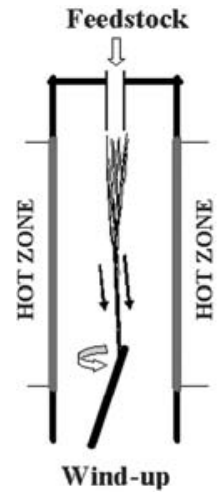

"Vertical"

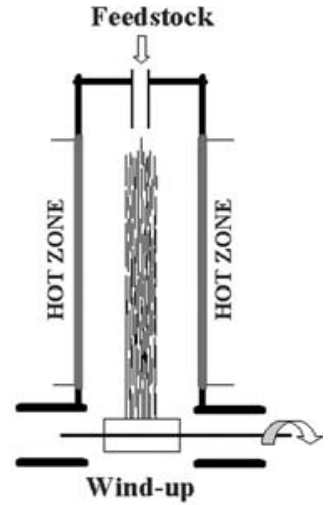

"Horizontal"
Fig. 7. Schematic diagrams of the process by which carbon nanotube fibers are spun directly from the CVD reaction zone. Two wind-up schemes are illustrated here, one to produce fiber, the other thin film

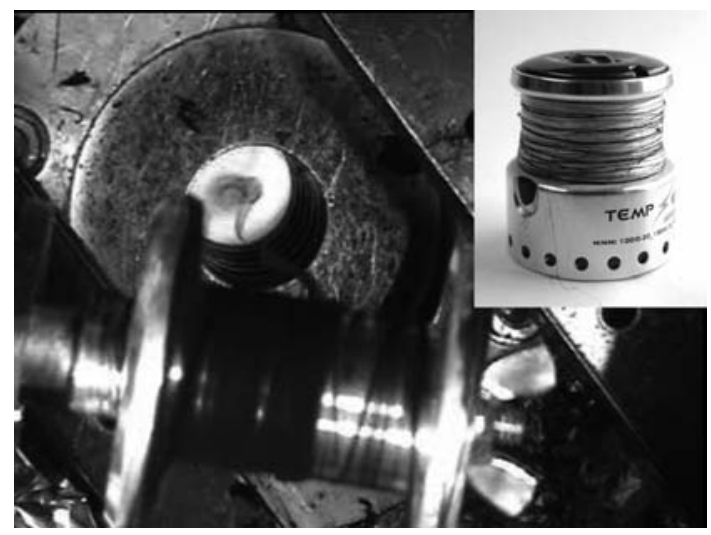

Fig. 8. Fiber being spun directly from the furnace via a gas valve that separates the hydrogen in the furnace from the air. The fiber condensation, at an intermediate level is achieved by the rotary motion of the gas. The inset shows a fiber after full condensation using acetone evaporation

ployed, or by subsequent twisting or wetting with an appropriate solvent. The process is enhanced through the addition of a sulfur-containing compound, typically thiophene, to the feed stock. Optimization of the process depends on the achievement of the right balance of feedstock composition, including catalyst and sulfur concentration, injection rate, hydrogen carrier gas flow rate, furnace temperature and wind-up speed $[148,149]$.

Fibers can be routinely spun with strengths of the order of $1 \mathrm{~N} /$ tex (if the specific gravity of the fiber is assumed to be unity, then these numbers equate to GPa) and if the conditions are taken to the limits of spinnability, then strengths of double this are seen. The elastic moduli are in the region of $70 \mathrm{~N} /$ tex [150] (Note: "tex" is a unit of measure for the linear mass den- 
sity of fibers, defined as the mass in grams per $1000 \mathrm{~m}$, i.e. 1 tex is equal to $10^{-6} \mathrm{~kg} / \mathrm{m}$ in S.I. units). Typical stress-strain curves of continuously spun material that has not been subjected to any post-treatment except for condensation are shown in Fig. 9. Very much higher values have been seen on short sections of the fiber, approaching $8 \mathrm{~N} /$ tex strength and $200 \mathrm{~N} /$ tex elastic modulus (values about three times those of Kevlar), which indicate not only the potential of the process but the directions for future development. The fibers are also tough, absorbing $60 \mathrm{~J} / \mathrm{g}$ at $\sim 10 \%$ extension. Adjustment of the process parameters can control the type of nanotubes comprising the fiber, whether they are single wall, thin wall or multiwall, and their mean diameter. The fibers with the best mechanical performance appear to comprise mainly double-wall nanotubes of unusually large diameter, so that many of them have undergone autocollapse to give "dog-bone" cross sections. The fibers also show a readiness to absorb polymer solutions, which opens up one of several routes to the further enhancement of their properties. Their electrical conductivity, without any optimization, is $0.3 \times 10^{6} \mathrm{~S} / \mathrm{m}$ and their thermal conductivity between 1000 and $1500 \mathrm{Wm}^{-1} \mathrm{~K}^{-1}$. As with the fibers made by dry spinning of nanotube forests, the fiber has yarn-like qualities. It is tough, flexible and has a knot strength of $80 \%$.

Of the three spinning methods described, the liquid route appears to produce the best-ordered fibers and quite stiff ones (up to $130 \mathrm{GPa}$ ), although the strengths were low, at around 0.2 GPa. Fibers dry-spun from multiwall nanotube forests have reported strengths up to $0.8 \mathrm{GPa}$, and stiffness of the order of $25 \mathrm{GPa}$. Fibers spun directly from the CVD reaction zone reach strengths considerably above $1 \mathrm{GPa}$, and stiffnesses equal to, or in excess of, the liquid-crystalline spun material, while much higher strengths and stiffness have been demonstrated on occasions, underlining the huge potential of the process. A key parameter is the length of the component nanotubes, which can now reach $>1 \mathrm{~mm}$ by "supergrowth" (Sect. 6.1). Electrical conductivity is also promising in pure dry-spun fibers, with values approaching $1 \%$ of copper, or $8 \%$ on a weight basis. Nanotube-polymer coagulation spun fibers show high energy absorption at fracture. Ultimately, the process that succeeds to win a substantial portion of the high-performance fiber market ( 2 B p.a.) will be the one that is the most industrially scalable, cheaper and better. See also the contribution in this volume by Endo et al.

\section{Organization on Surfaces}

The organization of carbon nanotubes on surfaces is an important issue toward many different future applications. Organization into vertical arrays (Sect. 6.1) may be especially important for field emitters in display technology (see contribution by Endo et al.), as well as a source for dry spinning of nanotube fibers (Sect. 5), whereas organization into horizontal arrays (Sects. 6.2 and 6.3) is a critical prerequisite for large-scale integration into 


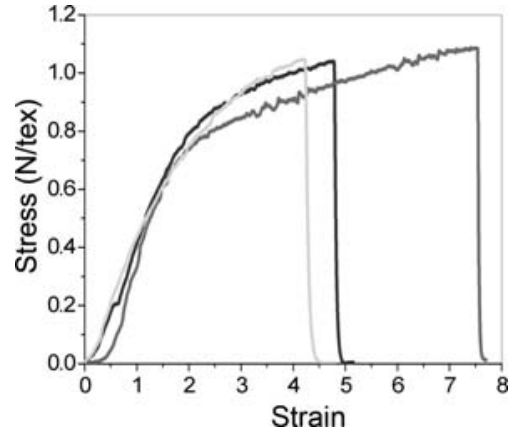

Fig. 9. Stress-strain curves from standard fibers that can be spun in the laboratory by the $\mathrm{km}$. Higher strengths can also be achieved

nanocircuits [151]. An important difference exists between the organization of SWNTs and MWNTs on surfaces, due to their different mechanical properties. MWNTs are relatively rigid, and their growth is more naturally directional. Therefore, significant progress in the organization of MWNTs on surfaces was achieved relatively earlier [152-154]. In contrast, SWNTs are relatively flexible, making their organization especially challenging. This section focuses on the recent progress in the organization of SWNTs on surfaces. Such organization can be achieved during nanotube formation by vertically or horizontally aligned growth (Sects. 6.1 and 6.3, respectively), or by organized assembly of pre-formed nanotubes (Sect. 6.2).

\subsection{Vertically Aligned Growth and Supergrowth}

With arc discharge or laser-ablation techniques, only tangled nanotubes mixed randomly with various impurities are obtainable. Research in CVD nanotube growth has also been initiated by the idea that aligned and ordered nanotube structures can be grown on surfaces with control, as first demonstrated by Ren and coworkers by growing large-diameter MWNTs forming oriented "forests" on glass substrates by PECVD [155], and by Fan and coworkers by CVD on uniform and catalytically patterned substrates [156]. Carbon nanotubes can self-assemble into aligned structures during CVD growth into vertically aligned films or patterns perpendicular to the substrate surface. The mechanism of nanotube self-orientation typically involves the nanotube base-growth mode substrates. During CVD growth, nanotubes interact with their neighbors via van der Waals forces to gain rigidity, which allows the nanotubes to self-orient and grow perpendicular to the substrate [7].

\subsubsection{Supergrowth}

Most growth methods based on CVD, such as those mentioned above, are seriously limited by the low activity and short life time of the catalyst. The resulting low catalytic activity of CNT synthesis has not only reduced the availability of SWNTs, but the dead catalysts remain in the as-grown material 
as impurities. Addition of a controlled amount of water vapor in the growth ambient can dramatically enhance the activity and lifetime of the catalysts and address these problems [157-161]. This new CVD approach denoted as "supergrowth", works best on substrates with catalytic nanoparticles. With the assistance of water, dense, vertical-standing, and aligned SWNT forests with millimeter-scale height can be synthesized. Figure 10a shows a 2.5-mm high SWNT forest that was grown in ten minutes [157]. The SWNT/catalyst weight ratio exceeds 50,000\%, more than 100 times higher than for other processes, illuminating the remarkable efficiency of water-assisted nanotube synthesis. A close examination (Fig. 10b) at the ledge of the SWNT forest illustrates that the nanotubes are well aligned vertically relative to the substrate. High-resolution transmission electron microscopy (TEM) studies (Fig. 10c) show that the nanotubes are clean SWNTs free from amorphous carbon. Low-resolution TEM studies (Fig. 10d) of the as-grown forest reveal the presence of only thin nanotubes and the absence of metallic particles and supporting materials that usually comprise a major constituent of as-grown SWNT material. Raman spectra showed clear radial breathing mode peaks that correspond well with the diameter of the SWNTs measured by TEM. Thermogravimetric analysis (TGA) on pure SWNT material using $\mathrm{N}_{2}$ gas with water shows that SWNT combustion in the presence of water starts at about $950^{\circ} \mathrm{C}$, indicating that the water does not oxidize or damage the nanotubes at the growth temperature.

Characterization of the structure of SWNT forests provided interesting insights into the growth mechanism [162]. For a typical SWNT forest, the SWNT area density is $5.2 \times 10^{11}$ tubes $/ \mathrm{cm}^{2}$, the mass density of the forest is $0.037 \mathrm{~g} / \mathrm{cm}^{3}$, the average SWNT size is $3.0 \mathrm{~nm}$, and the SWNT area density is $5.2 \times 10^{11}$ tubes $/ \mathrm{cm}^{2}$. The catalyst activity was estimated to be $84 \%( \pm 6 \%)$, the highest value ever reported for SWNT growth. On average, there exists one $3-\mathrm{nm}$ diameter SWNT in a substrate area of $190 \mathrm{~nm}^{2}$ and the average distance between tubes is $14 \mathrm{~nm}$. SWNTs occupy $3.6 \%$ of the total volume, and more that $96 \%$ is empty space. Interestingly, other SWNT forests synthesized by different methods and catalysts also show a similar sparseness, and the density of the CNTs is roughly in the range of a few to $10 \%$ [163]. The sparseness of the forest is thought to be essential for growing SWNT forests by CVD with root growth. This is because excessively high densities inhibit carbon diffusion to the catalysts (diffusion limited); whereas, for insufficiently high densities, the SWNTs would fail to grow vertically and would instead form a mat and quickly suffocate the catalysts as observed in normal CVD growth. With this sparseness, vast amounts of SWNTs can be grown on a substrate while not restricting the delivery of the carbon feedstock.

The SWNT forest structure can be easily removed from the substrate using, for example, a razor blade. After removal, the substrate is still catalytically active to grow SWNT forests again, which means that growth by a root-growth mode with catalysts remaining on the substrate after removal of 

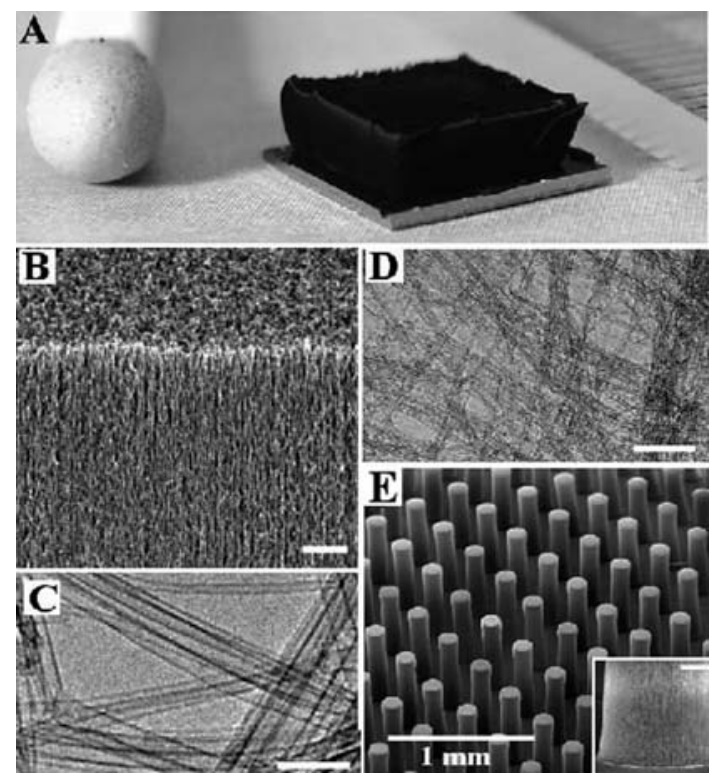

Fig. 10. (a) Picture of a single-walled carbon nanotube (SWNT) forest. (b) Scanning electron microscopy (SEM) image of the SWNT forest ledge. (c) Highresolution transmission electron microscopy (TEM) image of SWNTs. (d) A largearea TEM image. (e) SEM image of SWNT cylindrical pillars with $150 \mu \mathrm{m}$ radius, $250 \mu \mathrm{m}$ pitch, and $1 \mathrm{~nm}$ height. Inset, SEM image of a root of a pillar. Scale bar, $50 \mu \mathrm{m}[157]$

the forest. The high SWNT/catalyst weight ratio, the clean TEM images in Fig. 10, and the easy removal of the forests suggest that the as-grown SWNT material is highly pure. Thermogravimetric analysis (TGA) showed no measurable residue after heating above $750^{\circ} \mathrm{C}$, indicative of a carbon purity above $99 \%$. Quantitative elemental analysis with X-ray fluorescence spectrometry detected $0.013 \%$ Fe as the only impurity, meaning a carbon purity over $99.98 \%$, i.e., the purest SWNT material ever made.

Realization of large-scale organized SWNT structures of [157] desired shape and form is important for obtaining scaled-up functional devices. With the assistance of water, SWNTs grow easily from lithographically patterned catalyst islands into well-defined vertical-standing organized structures, as demonstrated by the large-scale arrays of macroscopic cylindrical pillars (Fig. 10e). The cross section of the SWNT structure corresponds well with the patterned catalyst (inset of Fig. 10e), and thus it is possible to fabricate arbitrary shapes of organized SWNT structures where the base is lithographically defined and the height is controlled by the growth time.

The effect of water can be revealed by [157] formulating a numerical growth model that describes the time evolution of the supergrowth [164]. The 
time evolution of the forest heights (yield) (Fig. 11a) showed that the growth rate gradually decreased over the subsequent $20 \mathrm{~min}$, and finally terminated with a height of $970 \mu \mathrm{m}$. The time evolution of the height $H(t)$ of the forest growth rate could be expressed, as $H(t)=\beta \tau_{0}\left[1-\exp \left(-t / \tau_{0}\right)\right]$, by assuming that the catalysts lose their activity in a similar fashion to radioactive decay. Important physical meaning can be assigned to the two fitting parameters: $\beta$ is the initial growth rate (IGR) and $\tau_{0}$ is the characteristic catalyst lifetime. Fitting the growth equation to the experimental time-evolution data yielded excellent agreement (Fig. 11a), and the fitting parameters were: IGR $(\beta)$ of $207 \mu \mathrm{m} / \mathrm{min}$ and lifetime $\left(\tau_{0}\right)$ of $4.74 \mathrm{~min}$, respectively. According to the growth equation, the product of the two fitting parameters, $\beta \tau_{0}$ gives the theoretical maximum height, $H_{\max }$, that was calculated to be $980 \mu \mathrm{m}$, matching well with the experimentally obtained height of $970 \mu \mathrm{m}$. A family of time-evolution data of the supergrowth was obtained at different ethylene flow rates at a fixed water level. From each growth equation curve, the initial growth rate $\beta$ and the characteristic catalyst lifetime $\tau_{0}$ was calculated and plotted as a function of the ethylene flow rate (Fig. 11b). Overall, the lifetime and IGR followed divergent trends, i.e., the IGR monotonically increases while the lifetime decreases with the ethylene level. The existence of these crossings among growth equation curves is a direct result of this divergent trend: slower initial growth with long lifetimes achieves higher maximum heights than rapid initial growth but with short lifetimes. Furthermore, despite this divergent trend, the theoretical maximum height (Fig. 11b, shown as a histogram), being the product of the two fitting parameters, $\beta \tau_{0}$ exhibits a peak representing the optimum ethylene flow rate for this water level. The divergent trends are easily explainable: 1 . IGR monotonically increases with ethylene flow rate because the carbon source for SWNT growth increases, 2. the lifetime decreases since the catalyst is poisoned faster by rapid accumulation of an amorphous-carbon coating, a factor known to kill the catalyst activity.

Further analysis was carried out by the two-dimensional mapping of the dependence of the lifetime, initial growth rate, and theoretical maximum height on water and ethylene. Rearranging the data into a plot of the maximum height as a function of the water/ethylene ratio revealed a clear trend. The maximum height initially increased with the water/ethylene ratio, peaked at a value of about $1 / 1000$, then decreased with further increase of the water/ethylene ratio. The water-assisted enhanced growth efficiency is reflected in the initial increase of the maximum height, and the peak represents the optimum growth conditions of the supergrowth. This result demonstrates the existence of a scaling relation between ethylene and water, and that the balance of the ethylene and water is the most critical factor for controlling the supergrowth. The same maximum height can be achieved by both a slow growth with a long lifetime and a fast growth with a short lifetime, given that the water/ethylene ratio is the same. Furthermore, the existence of this scaling law suggests that the catalysts consume a specific number of ethylene 

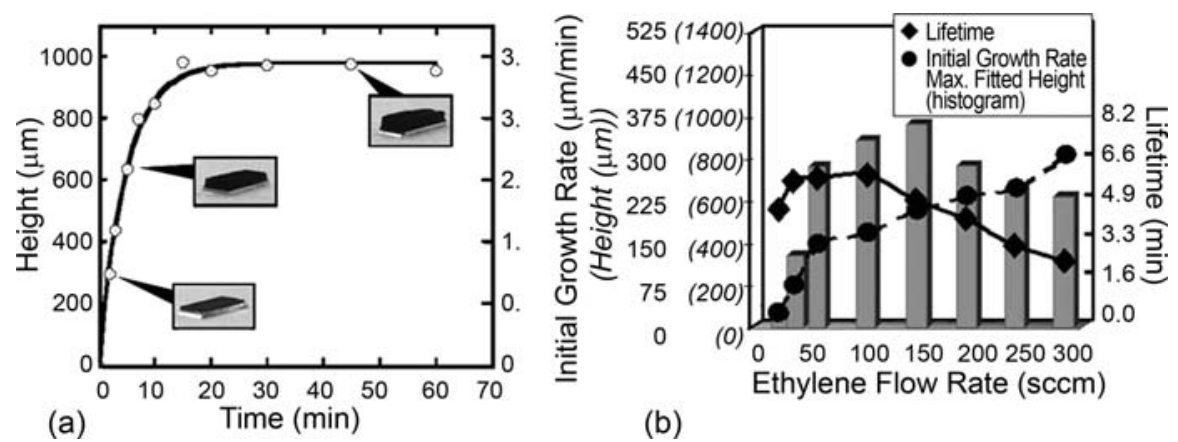

Fig. 11. Time evolution of SWNT forest growth. (a) Plot of the SWNT forest height as a function of the growth time. (b) Overlaid plots of the lifetime, initial growth rate, and maximum height (histogram), as derived from each growth curve [164]

and water molecules before they die, and this specific number is determined by the water/ethylene ratio. This implies that the rate-limiting process of the SWNT growth is the supply of carbon to catalysts from the gas phase.

Synthesis of catalyst-free DWNT forests with millimeter-scale height is also possible by using catalyst nanoparticles tailored to achieve maximum DWNT selectivity [165] (see more on DWNTs in the contribution by Pfeiffer et al.) Catalyst engineering is the key point for selective DWNT synthesis and was achieved by determining the optimum mean tube diameter for selective DWNT synthesis and by growing CNTs with this optimum mean diameter by precisely controlling the thickness of the Fe catalyst film. To understand and control CNT synthesis, the relationship between the tube type (single, double or multiwall) and diameter was studied by constructing a phase diagram (Fig. 12a) of the relative populations of SWNTs, DWNTs, MWNTs vs. the tube diameter. The phase diagram clearly shows that DWNTs occupy the majority of the nanotube population within a distinct diameter range sandwiched between the SWNT and MWNT regions. The existence of this distinct DWNT region affords selective DWNT growth via tuning the tube diameter into this DWNT region. Importantly, the mean CNT diameter was found to increase approximately linearly with the thickness of the Fe thin-film thickness (Fig. 12b). This finding enabled control of the mean CNT diameter accurately into the DWNT region. The maximum DWNT selectivity achieved with this approach was $85 \%$. This DWNT selectivity is one of the highest reported, and it is worth noting that it was achieved on an as-grown sample without any additional processes implemented to improve selectivity. Supergrowth was implemented to demonstrate highly efficient DWNT syntheses from these engineered catalyst particles, demonstrating that it is possible to 

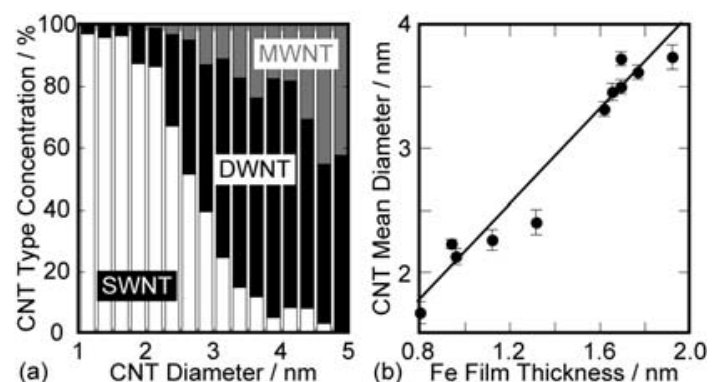

Fig. 12. Trends in CNT type and diameter. (a) Phase diagram of the relative concentration of SWNTs, double-wall carbon nanotubes (DWNTs) and multiwall carbon nanotubes as a function of the CNT diameter. (b) Plot of CNT mean diameter as a function of Fe film thickness [165]

grow DWNT forests with the same quality, yield and purity as the SWNT forests.

Generally, SWNT syntheses have been implemented on insulating substrates, such as Si wafers or quartz. In many cases, growths on conducting substrates are desired but have resulted in the formation of multiwalled carbon nanotubes (MWNTs) or graphite films. Ni-based alloys with $\mathrm{Cr}$ or Fe were found to possess both high durability to the CVD ambient and the ability to support highly efficient SWNT (DWNT) synthesis [166]. Successful syntheses of SWNT forests were achieved on various alloy substrates such as, Inconel 601, YEF 426, NiCr , YEF 50, SUS 310S, covering a wide range of $\mathrm{Ni}-\mathrm{Fe}-\mathrm{Cr}$ compositions (Fig. 13a). The growth product was mainly SWNTs with a selectivity of $95 \%$, and the growth yield and quality was comparable to the level of growth on Si wafers. To demonstrate an easy one-step assembly of CNT devices by this approach, a short DWNT forest was grown on a 1-cm diameter YEF 426 metal cathode (Fig. 13b) as a field electron emitter. The emission current increased exponentially with increasing electric field (Fig. 13c), following the Fowler-Nordheim equation. The spatial mapping of the emission current showed an excellent homogeneity that is evidence of good electrical contact between DWNTs and the grid substrate. Metal foils are much more economical and scalable than Si wafers or quartz substrates. Therefore, this approach would open up an economical route towards the mass production of SWNT and DWNT forests, and will facilitate flexible design of device architectures and fabrication processes for CNT devices.

\subsubsection{SWNT-Solid}

The intrinsic excellent properties of individual CNTs are frequently lost in macroscopic forms of CNTs. To address this matter, various CNT forms have been demonstrated ranging from fibers and yarns (Sect. 5), mats, vertically aligned CNTs (forests), powders, pellets, foams, and sheets. Yet, it is dif- 


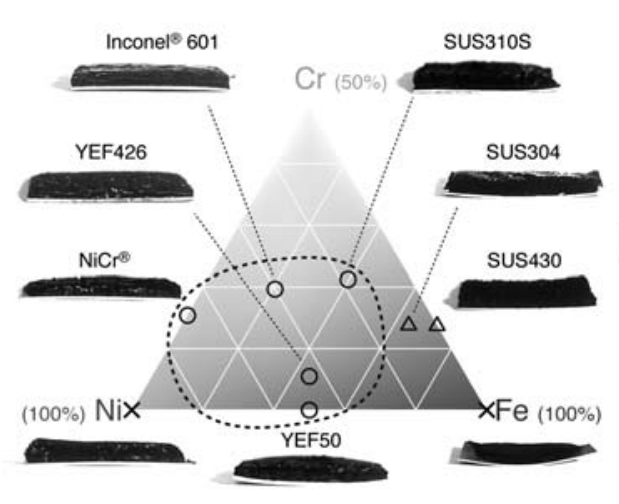

(a) (b)

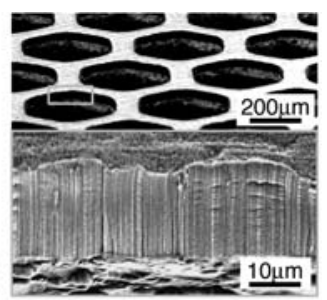

(c)

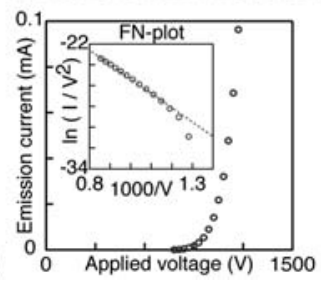

Fig. 13. (a) Correlation between components of $\mathrm{Ni}-\mathrm{Cr}-\mathrm{Fe}$ alloys and photographs of CNT forests grown on them. Symbols indicate the level of SWNT selectivity ( $\circ$ high selectivity; $\triangle$ medium selectivity; $\times$ low selectivity). (b) Double/YEF 426 as an electron field emitter. SEM images of a DWNT forest directly grown on a YEF 426 grid. (c) Emission current versus applied voltage profile (the inset shows a Fowler-Nordheim plot and fit) [166]

ficult to simultaneously retain the fundamental SWNT properties, such as high surface area and electrical conductivity on a macroscopic scale and have versatility in creating different shapes. A macroscopic bulk form of CNT material called the "SWNT solid" addresses this issue and has aligned densely packed SWNTs, while retaining the intrinsic properties of SWNTs [167]. When liquids are introduced into the sparse as-grown SWNT forest and dried, the surface tension of liquids, and the strong van der Waals interaction effectively "zippers" the SWNTs together in a nearly ideal graphitic spacing. Liquid-induced collapse shows a 4.5-fold decrease in the two lateral dimensions with no detectable change in the height, producing a $\sim 20$-fold increase in mass density (Fig. 14a). This indicates that alignment of the as-grown forest is critical in triggering an efficient liquid-induced collapse, which is understandable because the aligned nature of the growth provides an ideal condition for optimum van der Waals overlap. The initial $97 \%$ empty space in forests is here reduced to $\sim 50 \%$, and the intertube and innertube volumes become equivalent. The Vickers hardness of the SWNT solid increases over 70 -fold to $7-10$, which is comparable with that of highly oriented pyrolytic graphite (HOPG). The zippering action proceeds without damaging the tubes. The above characterization highlights the solidness and unity of the liquid-induced collapsed material, and thus this form was denoted as a "SWNT-solid".

The SWNT solid concept can be extended to create a variety of unique solid structures from as-grown forest material with diverse shapes by con- 
trolling the parameters that influence the collapse process, such as the forest aspect ratio, the initial liquid contact point, and substrate-forest interaction. For example, lithographically defined as-grown SWNT circular pillars with [167] a high aspect ratio collapsed from the tip creates well-defined and patterned SWNT solid needles with a high aspect ratio (Fig. 14b). Another approach to extend the ability to engineer the shape of the solid is to employ moderate external forces, such as very light pressure, to direct the direction of the collapse. For example, applying light pressure in one lateral direction suppresses collapse in one lateral dimension and creates a bar (Fig. 14d). Similar application of light pressure at a shearing angle to the alignment induces a vertical collapse, without any collapse in both lateral dimensions, results in a solid sheet (Fig. 14c). Because the SWNTs are directed to lie like a field of corn stalks flattened by a tractor, the solid sheets maintain a comparable degree of alignment of the forest. This approach is easily extendable to fabricate strongly adhered solid sheets on desired flat substrates without the use of bonding agents by simply transferring the forest onto a target substrate and implementing the collapse process. The ability to engineer the shapes of solids is beneficial for a multitude of applications requiring high surface area, high density, aligned, conducting, and flexible or rigid CNT material. The SWNT solids represent substantial progress towards producing a macroscopic scale, high-density SWNT material, engineered in both shape and structure, thus opening diverse functionality that is advantageous for numerous applications, such as energy storage.

\subsection{Organized Assembly of Preformed Nanotubes}

The controlled deposition of preformed nanotubes from solution onto a substrate with well-defined structures has been a topic of intense research for several years [168]. To be able to control the structure of deposited nanotubes, external forces, such as electrical, mechanical and chemical interactions, are needed to provide guidance toward the desired structures. One of the earliest examples of the controlled deposition of nanotubes on a substrate was developed by Smalley's group at Rice University, where surface patterns with chemically different functional groups are used to guide the structure of deposited nanotubes [169]. More recently, the affinity of nanotubes to $\mathrm{Al}_{2} \mathrm{O}_{3}$ was used as a way to guide nanotubes to specific locations to form nanotube devices in large numbers (Fig. 15) [170].

Surface patterns with different hydrophobic/hydrophilic functional groups is another common method to provide forces needed for guided deposition of nanotubes. A representative example for the use of such methods was developed by Rao et al. [171] where individual nanotubes or small bundles of nanotubes were deposited onto a substrate with precise control of their location and orientation (Fig. 16). More recently, Mirkin and coworkers [172, 173] used the edge of a hydrophilic pattern on a hydrophobic background to 


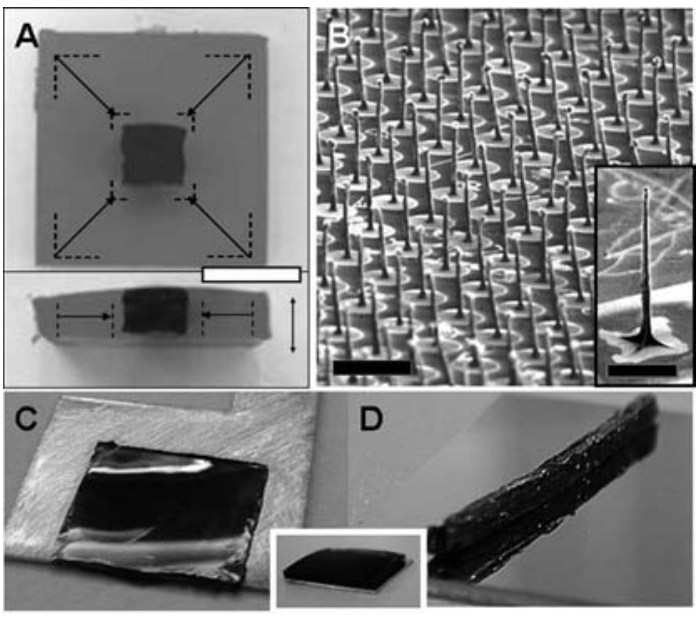

Fig. 14. (a) Overlaid pictures illustrating the decrease in lateral dimensions before (gray) and after (black) collapse. The double-ended arrow indicates tube alignment direction. (b) Engineerable shape: SEM image of an array of lithographically designed solid needles. Scale bar, $500 \mu \mathrm{m}$ Inset, magnified individual needle. Scale bar, $60 \mu \mathrm{m}$. (c) Flexible SWNT solid, engineered into a flattened sheet adhered to a copper sheet. Inset, Photograph of the as-grown forest. (d) SWNT solid, engineered into a rigid bar [167]

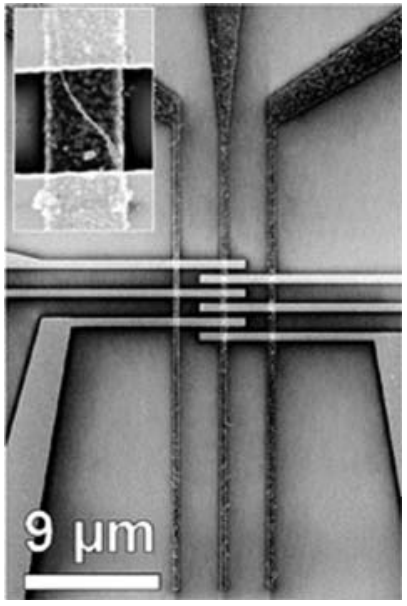

Fig. 15. SEM image of a CNT field-effect transistor [170]. Functionalized CNTs were assembled by their chemical affinity for the $\mathrm{Al}_{2} \mathrm{O}_{3}$ gate oxide, then contacted with evaporated gold source and drain electrodes [170] 
a)
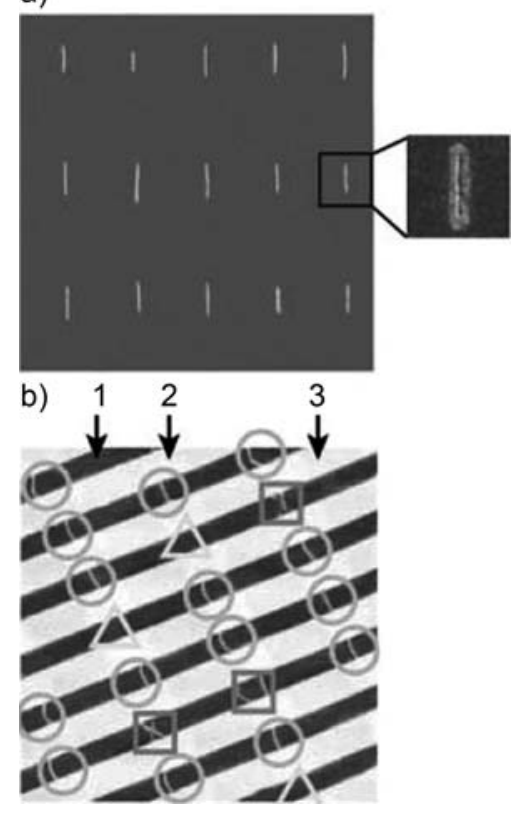

Fig. 16. AFM images showing largescale self-assembly of SWNTs: (a) Topography $\left(30 \times 30 \mu \mathrm{m}^{2}\right)$ of an array of individual nanotubes covering about $1 \mathrm{~cm}^{2}$ of a $\mathrm{Au}$ surface. The friction-force image (inset) shows a single nanotube (dark line), and the regions containing 2-mercaptoimidazole (bright area) and octadecyltrichlorosilane (ODT; used to passivate the $\mathrm{SiO}_{2}$ surface; dark area). (b) Topography $\left(20 \times 20 \mu^{2}\right)$ of an array of junctions with no nanotube $(\triangle)$, one nanotube $(\circ)$, or two nanotubes $(\square)$ covering an area of about $1 \mathrm{~cm}^{2}$. Arrows 1, 2, and 3 indicate ODT, 2mercaptoimidazole on $\mathrm{Au}$, and ODT on $\mathrm{Au}$, respectively [171]

organize nanotubes on a substrate into rings with well-controlled diameters and locations.

Electric and magnetic forces are also used to align nanotubes into desired structures. There are many reports on the formation of aligned nanotube structures under such forces. For example, Smalley and coworkers used a very strong magnetic field to form films with aligned nanotubes [174-178]. Mechanical force introduced by liquid flow was also demonstrated to align nanotubes into parallel structures [179]. However, it is the electric forces that showed the most promising advances in organizing nanotubes into desired structures. Both dc and ac dielectrophoresis have been used to deposit nanotubes from a suspension onto a substrate with good control of the alignment [59-61, 180-183]. Krupke and coworkers [60] used ac dielectrophoresis forces to precisely align nanotubes into parallel arrays of electrodes (Fig. 17), and provide a convenient way to make nanotube devices under good control. The same approach was used to separate metallic and semiconducting nanotubes [59] (Sect. 4).

Assembly of premade, chemically processed SWNTs on substrates is also a promising approach for the placement of densely aligned nanotubes. Langmuir-Blodgett (LB) films have been known for decades as an effective method for making well-organized organic monolayer patterns. Recently, Dai and coworkers [184] produced LB films of SWNTs on a large scale using HiPCO nanotubes as well as laser-ablation-grown nanotubes (Fig. 18). Suspensions of as-grown laser-ablation and HiPCO SWNTs in 1,2-dichloro- 

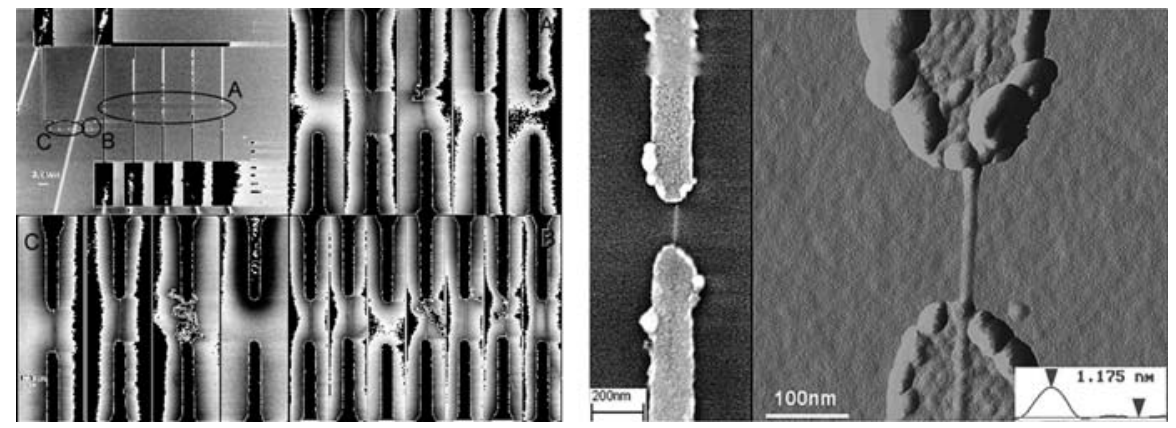

Fig. 17. SEM and AFM images of metallic nanotube bundles deposited on electrode arrays by dielectrophoresis [60] (the right-bottom corner inset shows a topographic section of a nanotube)

ethane (DCE) solutions of poly(m-phenylenevinylene-co-2,5-dioctoxy-p-phenylenevinylene) (PmPV) were prepared by sonication, ultra-centrifugation and filtration. The suspension contained mostly individual nanotubes well solubilized in DCE without free unbound PmPV. PmPV binds to SWNT sidewalls via $\pi$ stacking of its conjugated backbone and thus imparts solubility to nanotubes in an organic solvent. Importantly, DCE was found to be the only solvent in which PmPV-bound SWNTs remained stably suspended when free unbound PmPV molecules were removed. The PmPV-treated SWNTs exhibited no aggregation in DCE over several months. An LB film of SWNTs was made by adding SWNT DCE solutions to water in a LB trough to form a layer of SWNTs floating on a water surface upon vaporization of DCE. An aligned SWNT film was then formed by compressing the SWNTs on a water surface using two barriers. Such a film was then transferred to a substrate by simple dipping. AFM microscopy and Raman spectroscopy characterization revealed high-quality, densely aligned SWNTs (normal to the compression and substrate pulling direction) formed uniformly over large substrates for both HiPCO and laser-ablation-derived SWNTs. The height of the film relative to the nanotube-free regions of the substrate was $<2 \mathrm{~nm}$ under AFM, suggesting a monolayer of packed SWNTs [184].

Aligned SWNT LB monolayers on oxide substrates can be treated as carbon nanotube on insulator (CNT-OI) materials for patterning and integration into potential devices, much like how Si on insulator (SOI) has been used for electronics. Lithographic patterning techniques and oxygen plasma etching can be used to remove unwanted nanotubes and form patterned arrays for device integrations. The LB assembly of densely aligned SWNTs can be combined with chemical separation and selective chemical reaction methods to yield purely metallic or semiconducting SWNTs in a massively parallel configuration, useful for interconnection or high-speed transistor ap- 

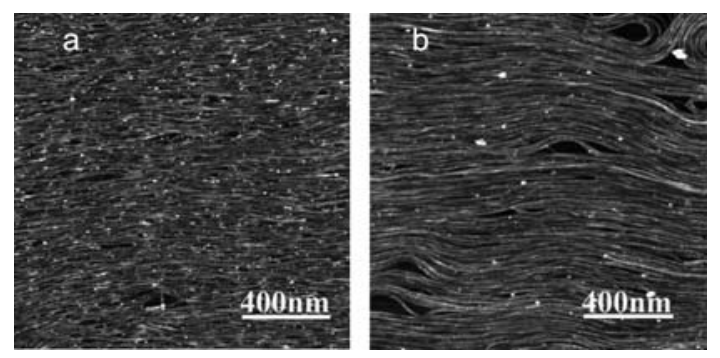

Fig. 18. Langmuir-Blodgett (LB) monolayer assembly of aligned SWNTs. (a) AFM image of a LB film of HiPCO SWNTs on a $\mathrm{SiO}_{2}$ substrate. (b) AFM image of a LB film of laser-ablation-grown SWNTs [184]

plications on a large scale. The method is generic in terms of the type of nanotube materials and substrates.

\subsection{Horizontally Aligned Growth}

An attractive approach for the organization of carbon nanotubes into horizontal arrays is the directed growth on surfaces by CVD [185], under the influence of an aligning force, such as an applied electric or magnetic field, the gas flow or the surface. One can envisage the creation of a variety of carbon nanotube arrays by defining the growth of the nanotubes on the surface in the form of a vector, having an origin, a direction, and a length. The origin can be defined by patterning the catalyst nanoparticles at specific locations, while the direction is defined by the aligning force, and the length is controlled by the growth time and rate. Successive or simultaneous steps of such "vectorial growth" [186] in different directions could yield different carbon nanotube architectures on surfaces.

\subsubsection{Field-Directed Growth}

Electric fields can orient carbon nanotubes owing to their large and highly anisotropic polarizability [187] (as seen in Sect. 4). This can be exploited for the alignment of carbon nanotubes during their growth. The growth of SWNTs suspended across lithographic ridges was observed by Dai and coworkers to be directed into parallel arrays by an electric field, which was applied by a pair of electrodes outside the ridges on a dielectric substrate during CVD growth [188], as shown in Fig. 19a. In the absence of an electric field, the SWNTs grow into random suspended networks (Fig. 19b).

Similar field-directed growth of SWNTs but lying on the surface was also observed by Joselevich et al. [186, 189] on thermally oxidized silicon wafers (Fig. 20a). In this case, the nanotubes are believed to grow up from the surface, and to be aligned by the electric field before they settle down and get 

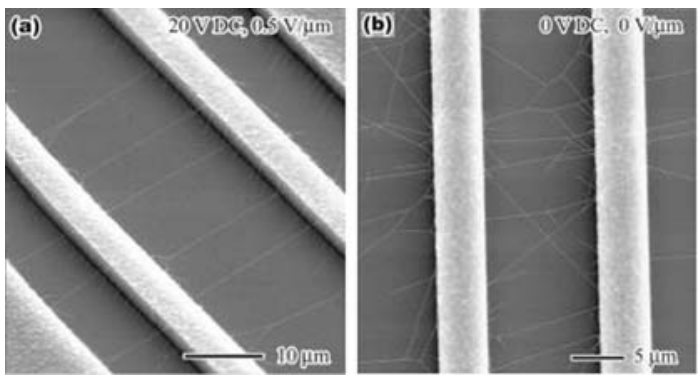

Fig. 19. Field-directed suspended SWNTs grown between two posts under an electric field (a) and in the absence of an electric field (b) [188]. The field intensity in (a) was $0.5 \mathrm{~V} / \mu \mathrm{m}$. The substrate is quartz. The distance between the poly-Si suspending ridges is $20 \mu \mathrm{m}$, and the distance between the outer poly-Si electrodes is $40 \mu \mathrm{m}$
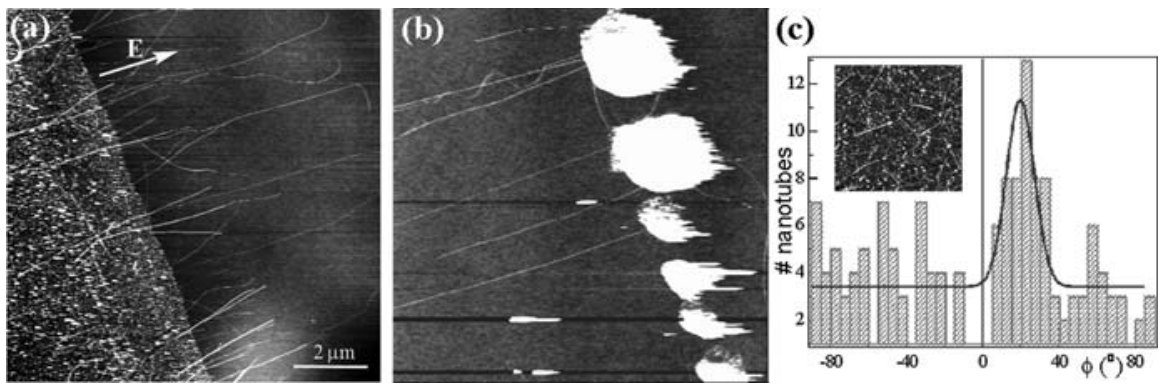

Fig. 20. Field-directed growth of SWNTs on oxidized Si wafers [186]. (a) Aligned array of SWNTs. The field intensity was $2 \times 10^{6} \mathrm{~V} / \mathrm{m}$. (b) Vectorial growth of SWNT arrays by field-directed growth from $\mathrm{Al}_{2} \mathrm{O}_{3}$-supported catalyst islands on the oxidized Si. (c) Bimodal angular distribution of field-directed short SWNTs $(L=200-$ $600 \mathrm{~nm}$ ). The solid line indicates a narrow Gaussian distribution around the direction of the field, which may be attributed to the metallic nanotubes, plus a broad distribution, which may be attributed to the semiconducting nanotubes. The inset (scale $2.5 \mu \mathrm{m}$ ) shows the partially aligned SWNTs

pinned to the surface by van der Waals forces. Vectorial growth of SWNTs from patterned islands of catalyst was used to produce ordered arrays of parallel 5-20 $\mu \mathrm{m}$ long SWNTs on oxidized silicon wafers (Fig. 20b) [186]. Interestingly, the angular distribution of short SWNTs $(L<200 \mathrm{~nm})$ grown under the same electric-field is not normal but bimodal (Fig. 20c). This can be attributed to the selective alignment of metallic carbon nanotubes, due to their higher polarizability compared to that of semiconducting ones.

Attempts to organize SWNTs into crossbar architectures by electric fielddirected growth in two dimensions actually led mainly to looped struc- 
tures [190]. This was attributed to the complex shape of the field lines, and showed the limitations of purely field-directed growth. Crossbar architectures using field-directed growth were successfully achieved by Joselevich and coworkers [191] in combination with surface-directed growth (vide infra). Field-directed growth was also used to grow SWNTs between electrodes to produce self-assembled field-effect transistors [192]. A different interesting approach combining field-directed growth with surface-directed growth is the oriented growth of SWNTs using lithographic alumina patterns, which created a local electric field due to static charging [193], without the need for electrodes and applied voltage. In this case, the SWNTs tend to lie perpendicular to the straight or curved edges of the alumina islands. Yet another approach for the generation of aligned arrays that is somehow related to electric-field-directed growth is the orientationally selective ablation of random networks with a planarly polarized laser [194]. SWNTs lying with a component parallel to the plane of polarization were selectively ablated, leaving on the surface only those nanotubes that are perpendicular to the plane of polarization.

Magnetic fields were also used to direct the growth of carbon nanotubes on surfaces in a few cases. Magnetic-field-directed growth of MWNTs was attributed to the interaction of the magnetic field with the ferromagnetic Fe catalyst [195]. Magnetic fields were also used to preorient the magnetite $\left(\mathrm{Fe}_{3} \mathrm{O}_{4}\right)$ nanoparticles from a magnetic bacterium, which were subsequently used as catalyst for CVD growth [196]. (See contribution by Kono et al.)

Overall, field-directed growth seems to be a useful tool for the organization of carbon nanotubes into horizontally aligned arrays. However, a practical limitation is that the electric field has to be generated by lithographic electrodes or charging islands, which impose size limitations and could interfere with other elements in functional devices. Also, silicon can significantly screen the electric field, producing complicated field lines, which are not parallel to the surface. Therefore, field-directed growth works fine on dielectric substrates, but is not ideal for large-scale organization on silicon wafers.

\subsubsection{Flow-Directed Growth}

Another promising approach to orient SWNTs is to use the feeding gas to align SWNTs along the flow direction. This method was originally discovered by Liu and coworkers [197-200] using a fast-heating CVD method, in which the Si wafer containing catalysts patterns were heated quickly to the reaction temperature by inserting the substrate into the center of a heated furnace. Figure 21 is a typical SEM image of the nanotubes from the new "fast-heating" process using $\mathrm{Fe} / \mathrm{Mo}$ catalyst nanoparticles and $\mathrm{CO} / \mathrm{H}_{2}$ as the feeding gas. AFM height measurements show that the diameters of the nanotubes range from $0.8 \mathrm{~nm}$ to $2.5 \mathrm{~nm}$, with an average diameter around $1.25 \mathrm{~nm}$. Note that not all the nanotubes under "fast-heating" are long and oriented. In the catalyst area there are still many short random nanotubes, relating to 


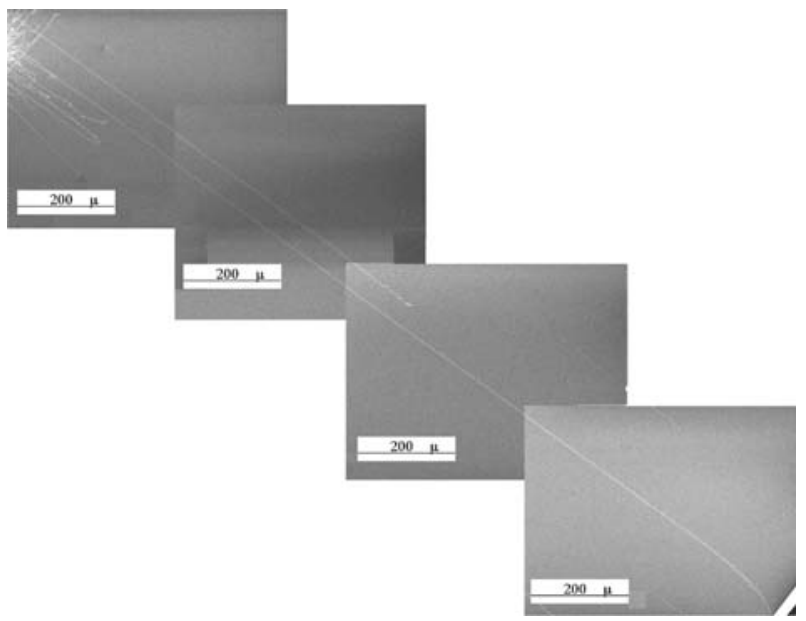

Fig. 21. SEM image of SWNTs grown with the flow-aligned CVD method

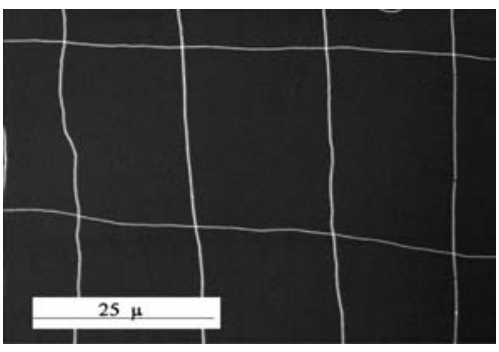

Fig. 22. SEM image of a $2 \mathrm{D}$ nanotube network grown using multistep growth [197]

different growth mechanisms, which will be discussed later. So, some of the nanotubes grown by the "fast-heating" process, using monodispersed Fe/Mo nanoparticles as a catalyst and $\mathrm{CO} / \mathrm{H}_{2}$ as the feeding gas, are long (up to a centimeter scale), well-oriented, and individual SWNTs. The orientation of nanotubes was found to be determined by the gas flow.

It has been proved that such a "fast-heating" process is a general method for growing superlong well-oriented SWNTs on a surface. Such superstructures of SWNTs can be generated by using different catalysts, other than $\mathrm{Fe} / \mathrm{Mo}$ nanoparticles (e.g., pure Fe nanoparticles, Fe/Pt nanoparticles, and water-soluble molecular Fe/Mo clusters), and different carbon sources other than $\mathrm{CO}$ (e.g., $\mathrm{CH}_{4}, \mathrm{CH}_{3} \mathrm{OH}$, etc.). It is believed that fast heating is also suitable for other SWNT growth systems. Such a big window provides a chance to control the SWNTs growth for different purposes.

As mentioned above, the long nanotubes from the "fast-heating" process are well oriented and the direction is determined by gas flow without using any external forces like a strong electric field. Figure 22 shows an SEM image of crossed nanotube arrays by a two-times patterning catalyst and a 
two-times growth using $\mathrm{Fe} / \mathrm{Mo}$ nanoparticles as catalysts and $\mathrm{CO} / \mathrm{H}_{2}$ as the feeding gas. A growth mechanism was proposed by the researchers, where a key component is that the nanotubes grow above the substrate surface. The fast-heating process was believed to cause convection of the gas flow due to the temperature difference between the substrate and feed gas. Such convection flow of the feed gas lifted the nanotubes upward and kept them floating and waving in the gas until they were caught by the laminar flow and came to the substrate. The actively growing nanotubes then floated in the feeding gas and grew along the flow direction. More recently, a variation of the fastheating method, and even methods without the need of the fast-heating step, were developed for the growth of aligned nanotubes with their growth directions controlled by the gas flow. For example, Burke and coworkers [201,202] discovered that the growth of nanotubes from catalysts deposited on a high platform fabricated on a flat Si wafer can grow long nanotubes not only along the gas flow but also against the direction of the gas flow. Additionally, no fast-heating step is needed in these studies. At first sight, the results seem to contradict the proposed growth mechanism. However, after a closer look, the nanotube alignment can be explained by the formation of reversed gas flow reflected by the high platform, and the platform serves as a mechanism to lift the catalysts and the growing nanotubes above the substrate, which are two of the key components of the mechanism: nanotubes growing above the substrate and the existence of a gas-flow current to direct the growth direction. More recently, Kim and coworkers [203] also demonstrated the use of a small quartz tube inside a large tube to achieve better alignment. It is believed that the small tubes can achieve better laminar flow of the feeding gas. $\mathrm{Li}$ and coworkers [204] also demonstrated the growth of well-aligned nanotubes with their directions controlled by gas flow using different catalysts. Overall, the use of gas flow to determine the growth directions of nanotubes not only produces aligned nanotubes, but the nanotubes grown in this way are almost always produced with extremely long lengths. A Raman study along individual long nanotubes revealed that the majority of the nanotubes showed a uniform radial breathing mode spectrum along their whole length, which is very encouraging for future use of the nanotubes in nanoelectronic devices.

\subsubsection{Surface-Directed Growth: "Nanotube Epitaxy"}

A promising new approach to carbon nanotube horizontal organization, which has developed rapidly during the last three years, is the growth of carbon nanotubes directed by well-defined crystal surfaces, or "nanotube epitaxy". Epitaxy generally refers to the "growth of a crystal of one material on the crystal base of another material in such a manner that its crystalline orientation is the same as that of the substrate". Epitaxy of inorganic materials is very important in the semiconductor industry, as well as in many other fields of technology. Molecular epitaxy [205], which refers to the formation of organized molecular layers on crystalline surfaces, has a more complex 
nature than the classical commensurate epitaxy of inorganic crystals, and has been recognized to exist in a hierarchy of different modes: Commensurate, semicommensurate (or "coincident") and incommensurate (or "orientational") epitaxy, taking place on atomically flat surfaces; ledge-directed epitaxy, taking place on vicinal surfaces, which are terminated with atomic steps; and graphoepitaxy, taking place on nanostructured surfaces. Joselevich and coworkers [206] have recently developed different nanotube analogs to some of these epitaxial modes, followed by other groups. These orientational heteroepitaxial modes of nanotube growth should not be confused with the continued growth of SWNTs [114-116] (Sects. 4.4 and 5.1), which may be considered as a mode of nanotube homoepitaxy.

Different phenomena that can now be considered as early manifestations of carbon nanotube epitaxy include the aligned electrophoretic deposition of SWNTs on highly oriented pyrolytic graphite C (0001) [43], the aligned growth of short SWNTs along the low-index directions of Si (100), Si (111) [207] and Au (111) [208] surfaces, the catalyst-free formation of SWNTs along or across step edges of $6 \mathrm{H}-\mathrm{SiC}$ (0001) [209], and the aligned dry deposition of SWNTs on GaAs (110) [210, 211]. In all these cases, however, the nanotubes lay in different directions and were often too short to be considered as organized arrays. The formation of highly aligned, unidirectional and dense arrays of long SWNTs on surfaces was first observed to form by CVD growth on low-quality C-plane sapphire [212]. The cheap C-plane sapphire wafers turned out to be miscut a few degrees off the Cplane, and the SWNT grew along the atomic steps of the vicinal $\alpha-\mathrm{Al}_{2} \mathrm{O}_{3}$ (0001) surfaces (vide infra). This mode of surface-directed growth could thus be related to ledge-directed epitaxy. Later, SWNTs were also shown to grow along well-defined directions of a variety of other crystal surfaces by different epitaxial modes. Following a rational order from smaller to larger aligning features, three different modes of carbon nanotube epitaxy have been identified so far [206]: lattice-directed epitaxy (by atomic rows), ledge-directed epitaxy (by atomic steps) and graphoepitaxy (by nanofacets).

\section{Lattice-Directed Nanotube Epitaxy by Atomic Rows}

Following the first reports of atomic-step-templated growth of SWNTs on miscut C-plane sapphire [212], two independent groups reported the observation of aligned SWNT growth on singular A-plane and R-plane sapphire, i.e., $\alpha-\mathrm{Al}_{2} \mathrm{O}_{3}(11 \overline{2} 0), \alpha-\mathrm{Al}_{2} \mathrm{O}_{3}(1 \overline{1} 02)$, respectively [213, 214], where the SWNTs

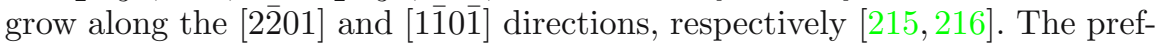
erential growth of SWNTs along these lattice directions was attributed to higher charge densities along these atomic rows due to electrostatic and van der Waals forces. The lattice-directed epitaxial growth of SWNTs on A-plane and R-plane sapphire was recently used for building field-effect transistors, and a nanotube-on-insulator technology of registry-free nanocircuits 
was proposed [217]. Lattice-directed epitaxial growth of SWNTs will probably take place on other atomically flat single-crystal substrates. Recently, for instance, SWNTs were observed to grow on $\mathrm{MgO}$ (001) preferentially along the [110] and [1ํㅣ directions [218].

\section{Ledge-Directed Nanotube Epitaxy by Atomic Steps}

SWNT produced by CVD on miscut C-plane sapphire were shown to grow along the $0.2-\mathrm{nm}$ high atomic steps of the vicinal $\alpha-\mathrm{Al}_{2} \mathrm{O}_{3}$ (0001) surfaces [212]. This atomic-step-templated mode of nanotube growth leads to the formation of dense arrays of highly parallel arrays of SWNTs, as shown in Fig. 23. The miscut is characterized by an inclination angle $\theta$ and an azimuth angle $\phi$, as defined in Fig. 23b. The degree of alignment correlates mainly with the miscut inclination angle $\theta$, while the straightness of the nanotubes correlates with the azimuth angle $\phi$ : When the step direction is

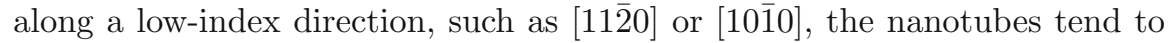
be straight. However, when the steps run along a high-index direction, then the nanotubes tend to be kinked, with straight segments parallel to the nearest low-index directions. Some nanotubes present sharp kinks of exactly $30^{\circ}$, which could be attributed to single pentagon-heptagon defects induced by kinked steps. These defects may involve structural changes along the nanotubes, constituting interesting metallic--semiconducting intramolecular junctions and superlattices. Single-nanotube Raman spectra from these samples exhibit a high intensity of D-band peaks, which indicates a significant loss of translational symmetry, consistent with this picture [219]. Polarized Raman measurements confirm the preferred orientation of the SWNTs along lowindex directions [220]. A "wake-growth mechanism" has been proposed [212], where the catalyst nanoparticle slides along the step leaving the nanotube behind as a wake. Recent AFM images of SWNTs grown on well-defined vicinal $\alpha-\mathrm{Al}_{2} \mathrm{O}_{3}(0001)$ surfaces (Fig. 23d), succeeded to resolve the densely packed atomic steps, and confirmed that the SWNTs continuously follow the atomic steps [220].

Ledge-directed nanotube epitaxy was recently observed also on miscut quartz [221]. In this case, the surface consists of vicinal $\alpha-\mathrm{SiO}_{2}(1 \overline{1} 01)$ with steps running along the $[\overline{2} 1 \overline{1} 0]$ direction. SWNTs grown on this surface by CVD form along the steps, similarly as on vicinal $\alpha-\mathrm{Al}_{2} \mathrm{O}_{3}$ (0001). The parallel arrays of SWNTs on quartz were used as thin-film transistors. Patterning of the catalyst allowed the formation of spatially selective arrays of SWNTs, and their integration into submillimeter thin-film transistors [222].

\section{Nanotube Graphoepitaxy by Nanofacets}

"Graphoepitaxy" is a mode of incommensurate epitaxy that generally refers to the orientation of crystals or periodic molecular assemblies by relief features of the substrate, such as steps or grooves, which can be significantly 

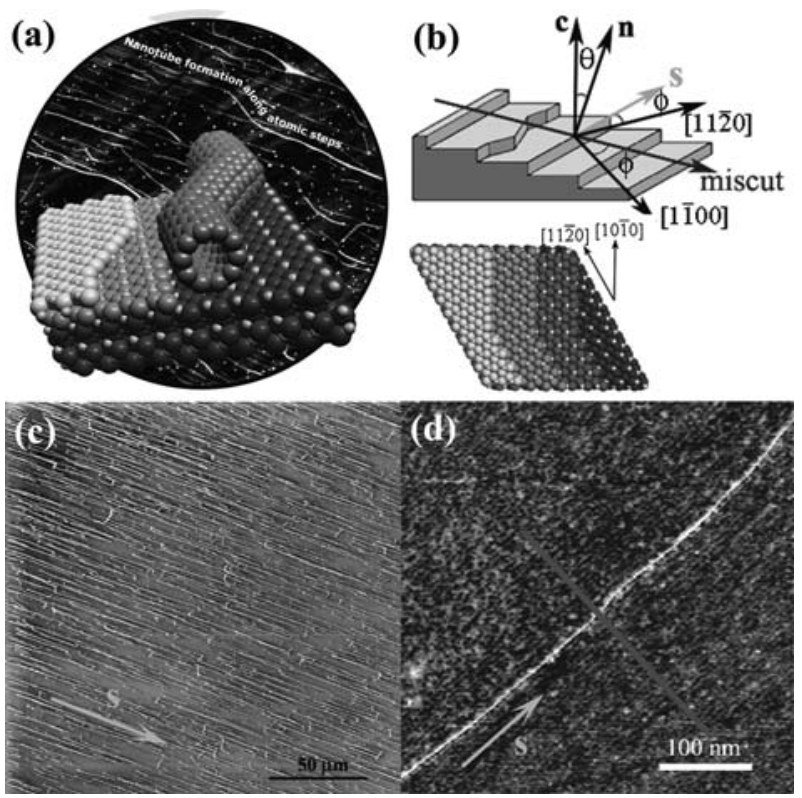

Fig. 23. Nanotube formation along the atomic steps of miscut C-plane sapphire, i.e., vicinal $\alpha-\mathrm{Al}_{2} \mathrm{O}_{3}$ (0001) [212]. (a) Model of a kinked SWNT grown along an atomic step on the background of an AFM topographic image of a real sample. (b) Definition of the step vector $\boldsymbol{s}=(\boldsymbol{c} / c) \times \boldsymbol{n}$, miscut inclination $\theta$, and miscut azimuth $\phi$, where $\boldsymbol{c}$ is the principal lattice vector and $\boldsymbol{n}$ is a unit vector normal to the surface. A top-view model of the vicinal surface is shown below. (c) Largescale SEM micrograph of SWNTs grown on a vicinal $\alpha-\mathrm{Al}_{2} \mathrm{O}_{3}$ (0001) surface. (d) High-resolution AFM topographic image providing a direct observation of the atomic steps on the surface, and a SWNT tightly following one of the steps [220]

larger than the lattice parameter [223, 224]. Graphoepitaxy of SWNTs was demonstrated on thermally annealed miscut C-plane sapphire [206]. When the vicinal $\alpha-\mathrm{Al}_{2} \mathrm{O}_{3}(0001)$ surfaces are annealed at $1100{ }^{\circ} \mathrm{C}$, the thermodynamically unstable atomic steps of $0.2 \mathrm{~nm}$ height bunch together into periodic nanosteps of $1.3 \mathrm{~nm}$ to $4 \mathrm{~nm}$ height. SWNTs grown by CVD on these periodically faceted surfaces generate a variety of morphologies, depending on the shape and surface energy of the nanofacets. As shown in Fig. 24, annealing of a C-plane sapphire miscut in different directions can yield nanofacets with a variety of morphologies (straight or sawtooth) and different surface energies. SWNTs produced on these nanofaceted surfaces (Fig. 25d) can have different morphologies (straight, wavy and ultrastraight). SWNTs grown on metastable nanofacets are especially straight, with angular deviations of less than $\pm 0.5^{\circ}$, for lengths of up to $100 \mu \mathrm{m}$ (Figs. 25a and b). This can be attributed to the high surface energy of these nanofacets, which makes them particularly sticky toward the nanotubes. 


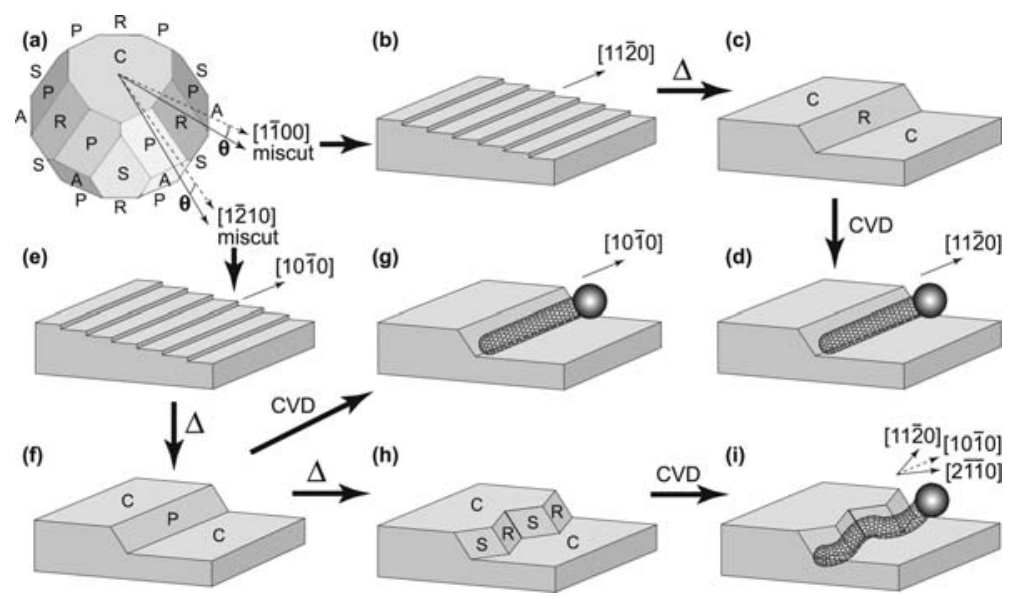

Fig. 24. Flow chart describing the formation of possible morphologies of carbon nanotube graphoepitaxy by miscut of C-plane sapphire, annealing, and CVD [206]. (a) Equilibrium shape of $\alpha-\mathrm{Al}_{2} \mathrm{O}_{3}$, with facets $\mathrm{C}\{0001\}, \mathrm{R}\{1 \overline{1} 02\}, \mathrm{S}\{1011\}$, $\mathrm{P}\{11 \overline{2} 3\}$, and $\mathrm{A}\{11 \overline{2} 0\}$, in order of increasing surface energy. The same drawing is used to show the different miscut directions. (b) Miscut toward [1100] produces a vicinal $\alpha-\mathrm{Al}_{2} \mathrm{O}_{3}$ (0001) surface with atomic steps along [1120]. (c) Annealing leads to R-faceted nanosteps. (d) SWNTs grow straight along [1120] (the ball represents the catalyst nanoparticle). (e) Miscut toward [12 10$]$ produces a vicinal $\alpha-\mathrm{Al}_{2} \mathrm{O}_{3}$ (0001) with atomic steps along [1010]. (f) Annealing initially leads to metastable P-faceted nanosteps. (g) SWNTs grow straight along [10̄10]. (h) Further annealing from (f) leads to sawtooth-shaped S/R-faceted nanosteps. (i) SWNTs grow loosely conformal to the sawtooth-shaped nanosteps, with segments along [11 $\overline{2} 0]$ and $[2 \overline{1} \overline{1} 0]$

\section{Nanotube Epitaxy Combined with External Forces}

Combinations of nanotube epitaxy with field-directed or flow-directed growth offer the possibility of generating more complex carbon nanotube patterns. For instance, crossbar architectures are especially desired for nanoelectronics, as they constitute the basis of most logic and memory elements in computing devices [225]. Crossbar arrays of single-wall carbon nanotubes were spontaneously produced in a single CVD step by simultaneous graphoepitaxy and field-directed growth, perpendicular to each other [191], as shown in Fig. 26. The two alignment mechanisms take place selectively on miscut Cplane sapphire and patterned amorphous $\mathrm{SiO}_{2}$ islands, respectively, without mutual interference, producing dense nanotube grids, with up to 12 junctions per $\mu \mathrm{m}^{2}$. This one-step method of orthogonal self-assembly may open up new possibilities for nanotube circuit integration. In principle, nanotube epitaxy should also be compatible with other external forces, such as in flow-directed growth (vide supra).

Judging by these examples, nanotube epitaxy seems to be rapidly evolving into an efficient approach for the organization of aligned arrays of SWNTs 

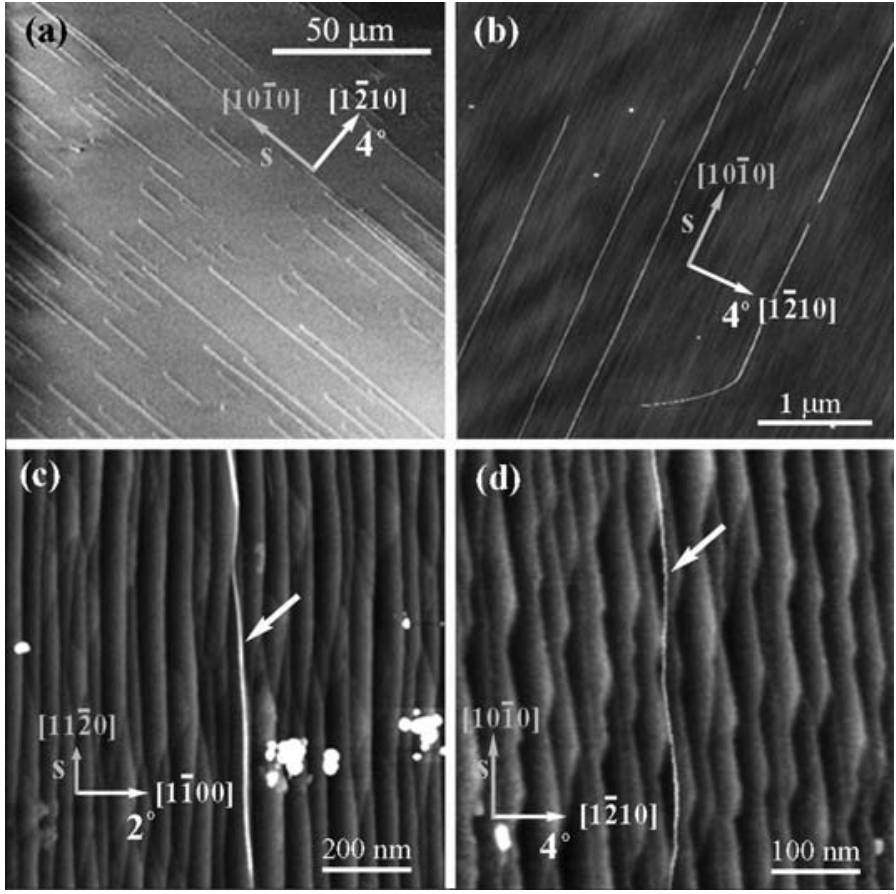

Fig. 25. Graphoepitaxial SWNTs on different annealed miscut C-plane sapphire [206]: (a) Straight nanosteps along [1010], as in Fig. 24g, observed by SEM. (b) AFM image of (a), showing the nanosteps. (c) Nanosteps along [1120], as in Fig. 24d. (d) Highly faceted sawtooth-shaped nanosteps along [1010], as in Fig. 24i

with a variety of morphologies, including highly straight, kinked, wavy and crossed. Some of these arrays have been already integrated into thin-film transistors. An intriguing open question that remains is whether all of these modes of nanotube epitaxy are indeed incommensurate, or may there be at least some degree of commensurism or registry between the surface lattice or the ledges, and the curved graphene lattice of the nanotubes. If this were the case, then one could expect a certain degree of control over the chirality and handedness of the nanotubes. Another question of greater practical implications toward future applications of nanotube epitaxy in nanoelectronics concerns the degree of atomic perfection and the electronic properties of the epitaxial nanotubes. Moreover, it is not yet clear whether or not the atomic steps and rows or nanofacets perturb the electronic properties of the nanotubes. The fact that these epitaxial substrates are bulk dielectric materials has also made their integration and electrical characterization difficult. A possible solution would be to develop a procedure for transferring the organized nanotubes from the epitaxial templates onto silicon wafers, or onto predefined 


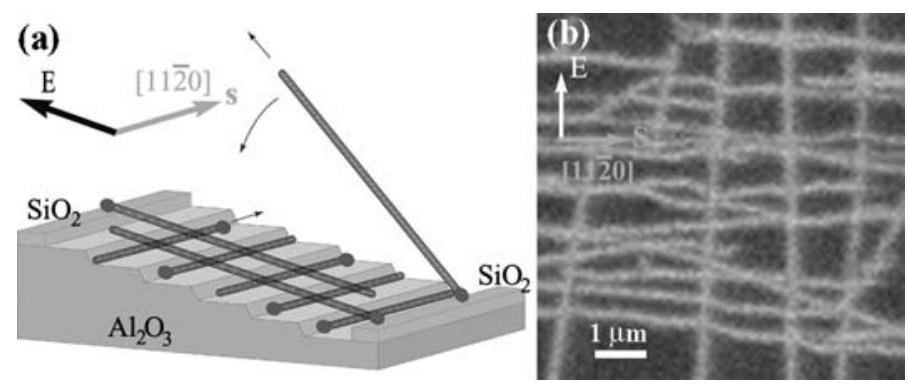

Fig. 26. Orthogonal self-assembly of SWNT crossbar architectures by simultaneous graphoepitaxy and field-directed growth, in a single step of CVD [191]. (a) Schematic representation of the process, showing the annealed miscut C-plane sapphire with nanosteps along the [11 $\overline{2} 0]$ direction, while the electric field is applied perpendicularly to the steps. The nanotubes originating from catalyst nanoparticles lying on the sapphire $\left(\mathrm{Al}_{2} \mathrm{O}_{3}\right)$ grow along the faceted nanosteps, while the nanotubes emerging from the catalyst on patterned amorphous $\mathrm{SiO}_{2}$ stripes grow freely without interacting with the surface, in a way that allows their alignment by the electric field, eventually falling across the nanotubes grown on the sapphire. (b) SEM image of a dense SWNT grid obtained by simultaneous graphoepitaxy and field-directed growth (miscut inclination angle $\theta=4^{\circ}$, field intensity $\left.E=2 \times 10^{6} \mathrm{~V} / \mathrm{m}\right)$

target structures. Progress in these directions may enable the realization of many applications.

\subsubsection{Patterned Growth on Surfaces}

Another important aspect of the organization of carbon nanotubes on surfaces is not only their orientation, but also their location. One approach to control the placement of SWNTs is to control the sites from which nanotubes are grown by patterning catalyst particles on a substrate [5]. The initial idea of such patterned growth was demonstrated with micrometer-scale islands of catalyst patterned on $\mathrm{SiO}_{2} / \mathrm{Si}$ wafers (Fig. 27a). Approximate location control of SWNTs (without orientation control) was obtained by chemical vapor deposition (CVD) growth of nanotubes from the catalyst islands. Since the initial patterned CVD growth demonstration, single-catalyst particle $(\sim$ $2 \mathrm{~nm}$ Fe clusters) patterning on substrates and orientation control of SWNTs grown by CVD have been actively pursued [226]. Single catalyst-nanoparticle patterning (Fig. 27b) for SWNTs growth by CVD was recently achieved (Fig. 27c). 


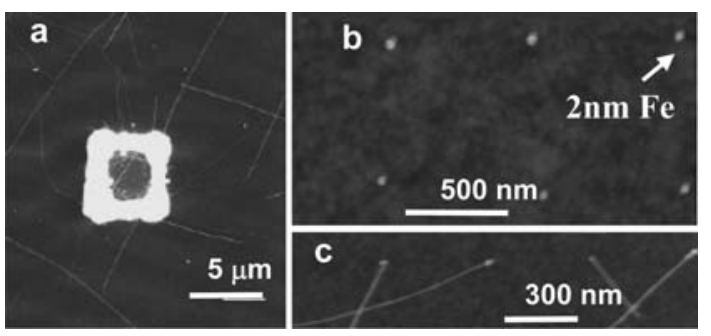

Fig. 27. Patterned growth of SWNTs. (a) Previous growth from micrometer-sized catalyst island (white structure) [5]. (b) AFM image of a recently made regular array of individual Fe clusters [226]. (c) SWNTs grown from the single-particle array

\section{Summary and Outlook}

Mass production, selective production, and organization of carbon nanotubes continue to be major synthetic challenges toward future applications. Significant advances during the last five years include the development of new CVD methods for mass production and for aligned growth on surfaces, both vertically and horizontally. Mass production and vertically aligned growth by CVD have been significantly boosted by the discovery of "supergrowth", adding small amounts of water to the CVD precursor or using alcohol as the carbon feedstock, to inhibit the formation of amorphous carbon, and thus to enhance the formation of clean carbon nanotubes. A fundamental key to further increase the control of carbon nanotube synthesis is understanding the different mechanisms of formation of carbon nanotubes, which determine their structure, electronic properties and arrangement. Since the mechanisms of CVD growth are not yet fully understood, further mechanistic insight will probably have an impact on the yield, purity and selectivity of CVD growth. An important development in this direction has been the direct observation of nanotube growth under the TEM [227].

We have seen promising advances in the selective production of carbon nanotubes, as well as in the continued growth of carbon nanotubes with conservation of their initial $(n, m)$ structure. Single-wall carbon nanotubes have been sorted by length, electronic type and diameter using a variety of physical and chemical methods. Combinations of these methods promise to enable the production of nanotube fractions with progressively narrower $(n, m)$ distribution. However, the selective production of pure nanotube samples with a unique $(n, m)$ structure is a challenge yet to be met.

An important synthetic advance toward application of carbon nanotubes as structural and textile materials has been the development of new methods for spinning carbon nanotube fibers, either from liquid suspension, from dry forests, or directly from the CVD zone during growth. The mechanical properties of these carbon nanotube fibers are remarkable, but are still inferior to 
those of individual carbon nanotubes. Approaching the nanoscopic properties at a macroscopic scale will probably require a better control, not only of the nanotube length and alignment, but also of the nanotube-nanotube interactions. Textile processing of carbon nanotubes into ultrastrong or "smart" clothing could be an exciting outcome to be seen soon.

Finally, the organization of carbon nanotubes into horizontally aligned arrays on surfaces, which continues to be a major challenge toward integration into nanocircuits, has been significantly advanced by the development fielddirected, flow-directed and surface-directed growth. Flow-directed growth has allowed the controlled production of macroscopically long carbon nanotubes on surfaces. More recently, aligned growth by orientational epitaxy on welldefined crystal surfaces, directed by atomic steps, nanofacets or atomic rows, has opened up new ways of organizing nanotubes on surfaces into perfectly aligned arrays, as well as more complex morphologies. In addition to the control of nanotube orientation, there is still a need for better control of the nanotube location. This requires a reliable method for patterning catalyst nanoparticles that nucleate carbon nanotubes with a $100 \%$ yield. Thanks to encouraging advances in this direction, the combination of patterned growth with orientational control is a promising prospect, which could greatly advance nanotube electronics in the next few years.

\section{References}

[1] T. W. Ebbesen, P. M. Ajayan: Large-scale synthesis of carbon nanotubes, Nature 358, 220-222 (1992) 102, 103

[2] D. S. Bethune, C. H. Kiang, M. DeVries, G. Gorman, R. Savoy, J. Vazquez, R. Beyers: Cobalt-catalysed growth of carbon nanotubes with single-atomiclayer walls, Nature 363, 605-607 (1993) 102, 103

[3] A. Thess, R. Lee, P. Nikolaev, H. J. Dai, P. Petit, J. Robert, C. H. Xu, Y. H. Lee, S. G. Kim, A. G. Rinzler, D. T. Colbert, G. E. Scuseria, D. Tomanek, J. E. Fischer, R. E. Smalley: Crystalline ropes of metallic carbon nanotubes, Science 273, 483-487 (1996) 102, 103, 105

[4] H. Dai, A. G. Rinzler, A. Thess, P. Nikolaev, D. T. Colbert, R. E. Smalley: Single-wall carbon nanotubes produced by metal-catalyzed disproportionation of carbon monoxide, Chem. Phys. Lett. 260, 471-475 (1996) 102, 104, 105

[5] J. Kong, H. Soh, A. Cassell, C. F. Quate, H. Dai: Synthesis of individual single-walled carbon nanotubes on patterned silicon wafers, Nature 395, 878881 (1998) 102, 104, 147, 148

[6] J. Hafner, M. Bronikowski, B. Azamian, P. Nikolaev, D. Colbert, R. Smalley: Catalytic growth of single-wall carbon nanotubes from metal particles, Chem. Phys. Lett. 296, 195-202. (1998) 102, 104

[7] H. Dai: Carbon nanotubes: opportunities and challenges, Surf. Sci. 500, 218241 (2002) 103, 104, 126

[8] H. Dai: Carbon nanotubes: synthesis, integration, and properties, Acc. Chem. Res. 35, 1035-1044 (2002) 103, 104 
[9] H. Dai, J. Kong, C. Zhou, N. Franklin, T. Tombler, A. Cassell, S. Fan, M. Chapline: Controlled chemical routes to nanotube architectures, physics and devices, J. Phys. Chem. 103, 11246-11255 (1999) 103, 104

[10] J. Kong, A. M. Cassell, H. Dai: Chemical vapor deposition of methane for single-walled carbon nanotubes, Chem. Phys. Lett. 292, 567-574 (1998) 104

[11] A. Cassell, J. Raymakers, J. Kong, H. Dai: Large scale single-walled nanotubes by CVD synthesis, J. Phys. Chem. 103, 6484-6492 (1999) 104

[12] M. Su, B. Zheng, J. Liu: A scalable CVD method for the synthesis of singlewalled carbon nanotubes with high catalyst productivity, Chem. Phys. Lett. 322, 321-326 (2000) 104

[13] P. Nikolaev, M. J. Bronikowski, R. K. Bradley, F. Rohmund, D. T. Colbert, K. A. Smith, R. E. Smalley: Gas-phase catalytic growth of single-walled carbon nanotubes from carbon monoxide, Chem. Phys. Lett. 313, 91-97 (1999) 104

[14] B. Kitiyanan, W. E. Alvarez, J. H. Harwell, D. E. Resasco: Controlled production of single-wall carbon nanotubes by catalytic decomposition of $\mathrm{CO}$ on bimetallic Co-Mo catalysts, Chem. Phys. Lett. 317, 497-503 (2000) 104

[15] S. Maruyama, R. Kojima, Y. Miyauchi, S. Chiashi, M. Kohno: Lowtemperature synthesis of high-purity single-walled carbon nanotubes from alcohol, Chem. Phys. Lett. 360, 229-234 (2002) 104

[16] Y. M. Li, D. Mann, M. Rolandi, W. Kim, A. Ural, S. Hung, A. Javey, J. Cao, D. W. Wang, E. Yenilmez, Q. Wang, J. F. Gibbons, Y. Nishi, H. J. Dai: Preferential growth of semiconducting single-walled carbon nanotubes by a plasma enhanced CVD method, Nano Lett. 4, 317-321 (2004) 105, 106

[17] G. F. Zhong, T. Iwasaki, K. Honda, Y. Furukawa, I. Ohdomari, H. Kawarada: Low temperature synthesis of extremely dense and vertically aligned singlewalled carbon nanotubes, Jpn. J. App. Phys. 1 44, 1558-1561 (2005) 105

[18] G. Y. Zhang, D. Mann, L. Zhang, A. Javey, Y. M. Li, E. Yenilmenz, Q. Wang, J. McVittie, Y. Nishi, J. Gibbons, H. Dai: Ultra-high-yield growth of vertical single-walled carbon nanotubes: Hidden roles of hydrogen and oxygen, Proc. Nat. Acad. Sci. 102, 16141-16145 (2005) 105

[19] W. L. Wang, X. D. Bai, Z. Xu, S. Liu, E. G. Wang: Low temperature growth of single-walled carbon nanotubes: Small diameters with narrow distribution, Chem. Phys. Lett. 419, 81-85 (2006) 105

[20] T. Kato, R. Hatakeyama, K. Tohji: Diffusion plasma chemical vapour deposition yielding freestanding individual single-walled carbon nanotubes on a silicon-based flat substrate, Nanotechnol. 17, 2223-2226 (2006) 105

[21] A. V. Melechko, V. I. Merkulov, T. E. McKnight, M. A. Guillorn, K. L. Klein, D. H. Lowndes, M. L. Simpson: Vertically aligned carbon nanofibers and related structures: Controlled synthesis and directed assembly, J. Appl. Phys. 97, $041301(2005) \quad 105$

[22] C. Journet, W. K. Maser, P. Bernier, A. Loiseau, M. L. delaChapelle, S. Lefrant, P. Deniard, R. Lee, J. E. Fischer: Large-scale production of singlewalled carbon nanotubes by the electric-arc technique, Nature 388, 756-758 (1997) 105

[23] P. Nikolaev, M. J. Bronikowski, R. K. Bradley, F. Rohmund, D. T. Colbert, K. A. Smith, R. E. Smalley: Gas-phase catalytic growth of single-walled carbon nanotubes from carbon monoxide, Chem. Phys. Lett. 313, 91-97 (1999) 105 
[24] Y. Li, S. Peng, D. Mann, J. Cao, R. Tu, K. J. Cho, H. Dai: On the origin of preferential growth of semiconducting single-walled carbon nanotubes, J. Phys. Chem. B 109, 6968-6971 (2005) 107

[25] G. Y. Zhang, P. F. Qi, X. R. Wang, Y. R. Lu, X. L. Li, R. Tu, S. Bangsaruntip, D. Mann, L. Zhang, H. J. Dai: Selective etching of metallic carbon nanotubes by gas-phase reaction, Science 314, 974-977 (2006) 107, 115

[26] S. M. Bachilo, L. Balzano, J. E. Herrera, F. Pompeo, D. E. Resasco, R. B. Weisman: Narrow (n,m)-Distribution of Single-Walled Carbon Nanotubes Grown Using a Solid Supported Catalyst, J. Am. Chem. Soc. 125, 11186-11187 (2003) 107

[27] G. Lolli, L. A. Zhang, L. Balzano, N. Sakulchaicharoen, Y. Q. Tan, D. E. Resasco: Tailoring $(\mathrm{n}, \mathrm{m})$ structure of single-walled carbon nanotubes by modifying reaction conditions and the nature of the support of CoMo catalysts, J. Phys. Chem. B 110, 2108-2115 (2006) 107

[28] T. J. Park, S. Banerjee, T. Hemraj-Benny, S. S. Wong: Purification strategies and purity visualization techniques for single-walled carbon nanotubes, J. Mater. Chem. 16, 141-154 (2006) 107, 108

[29] R. C. Haddon, J. Sippel, A. G. Rinzler, F. Papadimitrakopoulos: Purification and separation of carbon nanotubes, MRS Bull. 29, 252-259 (2004) 107, 108, 109, 110

[30] T. W. Ebbesen, P. M. Ajayan, H. Hiura, K. Tanigaki: Purification of Nanotubes, Nature 367, 519-519 (1994) 108

[31] J. L. Zimmerman, R. K. Bradley, C. B. Huffman, R. H. Hauge, J. L. Margrave: Gas-phase purification of single-wall carbon nanotubes, Chem. Mater. 12, 1361-1366 (2000) 108

[32] J. M. Moon, K. H. An, Y. H. Lee, Y. S. Park, D. J. Bae, G. S. Park: Highyield purification process of singlewalled carbon nanotubes, J. Phys. Chem. B 105, 5677-5681 (2001) 108

[33] Z. J. Shi, Y. F. Lian, F. H. Liao, X. H. Zhou, Z. N. Gu, Y. G. Zhang, S. Iijima: Purification of single-wall carbon nanotubes, Solid State Commun. 112, 3537 (1999) 108

[34] I. W. Chiang, B. E. Brinson, A. Y. Huang, P. A. Willis, M. J. Bronikowski, J. L. Margrave, R. E. Smalley, R. H. Hauge: Purification and characterization of single-wall carbon nanotubes (SWNTs) obtained from the gas-phase decomposition of CO (HiPco process), J. Phys. Chem. B 105, 8297-8301 (2001) 108

[35] Y. Q. Xu, H. Q. Peng, R. H. Hauge, R. E. Smalley: Controlled multistep purification of single-walled carbon nanotubes, Nano Lett. 5, 163-168 (2005) 108

[36] I. W. Chiang, B. E. Brinson, R. E. Smalley, J. L. Margrave, R. H. Hauge: Purification and characterization of single-wall carbon nanotubes, J. Phys. Chem. B 105, 1157-1161 (2001) 108

[37] A. C. Dillon, T. Gennett, K. M. Jones, J. L. Alleman, P. A. Parilla, M. J. Heben: A simple and complete purification of single-walled carbon nanotube materials, Adv. Mater. 11, 1354-1358 (1999) 108

[38] E. Dujardin, T. W. Ebbesen, A. Krishnan, M. M. J. Treacy: Purification of single-shell nanotubes, Adv. Mater. 10, 611-613 (1998) 108 
[39] D. Chattopadhyay, I. Galeska, F. Papadimitrakopoulos: Complete elimination of metal catalysts from single wall carbon nanotubes, Carbon 40, 985-988 (2002) 108

[40] M. Monthioux, B. W. Smith, B. Burteaux, A. Claye, J. E. Fischer, D. E. Luzzi: Sensitivity of single-wall carbon nanotubes to chemical processing: an electron microscopy investigation, Carbon 39, 1251-1272 (2001) 108

[41] A. G. Rinzler, J. Liu, H. Dai, P. Nikolaev, C. B. Huffman, F. J. RodriguezMacias, P. J. Boul, A. H. Lu, D. Heymann, D. T. Colbert, R. S. Lee, J. E. Fischer, A. M. Rao, P. C. Eklund, R. E. Smalley: Large-scale purification of single-wall carbon nanotubes: process, product, and characterization, Appl. Phys. A-Mater. 67, 29-37 (1998) 108

[42] C. Bower, A. Kleinhammes, Y. Wu, O. Zhou: Intercalation and partial exfoliation of single-walled carbon nanotubes by nitric acid, Chem. Phys. Lett. 288, 481-486 (1998) 108

[43] J. Liu, A. G. Rinzler, H. J. Dai, J. H. Hafner, R. K. Bradley, P. J. Boul, A. Lu, T. Iverson, K. Shelimov, C. B. Huffman, F. Rodriguez-Macias, Y. S. Shon, T. R. Lee, D. T. Colbert, R. E. Smalley: Fullerene pipes, Science 280, 12531256 (1998) 108, 111, 115, 142

[44] Y. L. Yang, L. M. Xie, Z. Chen, M. H. Liu, T. Zhu, Z. F. Liu: Purification and length separation of single-walled carbon nanotubes using chromatographic method, Synth. Met. 155, 455-460 (2005) 109, 111, 115

[45] X. Y. Huang, R. S. McLean, M. Zheng: High-resolution length sorting and purification of DNA-wrapped carbon nanotubes by size-exclusion chromatography, Anal. Chem. 77, 6225-6228 (2005) 109, 111, 115

[46] S. Niyogi, H. Hu, M. A. Hamon, P. Bhowmik, B. Zhao, S. M. Rozenzhak, J. Chen, M. E. Itkis, M. S. Meier, R. C. Haddon: Chromatographic purification of soluble single-walled carbon nanotubes (s-SWNTs), J. Am. Chem. Soc. 123, 733-734 (2001) 109

[47] A. P. Yu, E. Bekyarova, M. E. Itkis, D. Fakhrutdinov, R. Webster, R. C. Haddon: Application of centrifugation to the large-scale purification of electric arc-produced single-walled carbon nanotube, J. Am. Chem. Soc. 128, 99029908 (2006) 109

[48] H. B. Jia, Y. F. Lian, M. O. Ishitsuka, T. Nakahodo, Y. Maeda, T. Tsuchiya, T. Wakahara, T. Akasaka: Centrifugal purification of chemically modified single-walled carbon nanotube, Sci. Technol. Adv. Mater. 6, 571-581 (2005) 109

[49] R. Krupke, F. Hennrich: Separation techniques for carbon nanotubes, Adv. Eng. Mater. 7, 111-116 (2005) 109, 110

[50] M. J. O'Connell, S. M. Bachilo, C. B. Huffman, V. C. Moore, M. S. Strano, E. H. Haroz, K. L. Rialon, P. J. Boul, W. H. Noon, C. Kittrell, J. P. Ma, R. H. Hauge, R. B. Weisman, R. E. Smalley: Band gap fluorescence from individual single-walled carbon nanotubes, Science 297, 593-596 (2002) 109

[51] B. L. Chen, J. P. Selegue: Separation and characterization of single-walled and multiwalled carbon nanotubes by using flow field-flow fractionation, Anal. Chem. 74, 4774-4780 (2002) 111, 115

[52] G. S. Duesberg, M. Burghard, J. Muster, G. Philipp, S. Roth: Separation of carbon nanotubes by size exclusion chromatography, Chem. Commun. pp. 435-436 (1998) 111, 115, 118 
[53] K. Arnold, F. Hennrich, R. Krupke, S. Lebedkin, M. M. Kappes: Length separation studies of single walled carbon nanotube dispersions, Phys. Stat. Sol. B 243, 3073-3076 (2006) 111, 115

[54] G. S. Duesberg, J. Muster, V. Krstic, M. Burghard, S. Roth: Chromatographic size separation of single-wall carbon nanotubes, Appl. Phys. A-Mater. 67, 117-119 (1998) 111, 115

[55] G. S. Duesberg, W. Blau, H. J. Byrne, J. Muster, M. Burghard, S. Roth: Chromatography of carbon nanotubes, Synthetic Met. 103, 2484-2485 (1999) 111,115

[56] E. Farkas, M. E. Anderson, Z. H. Chen, A. G. Rinzler: Length sorting cut single wall carbon nanotubes by high performance liquid chromatography, Chem. Phys. Lett. 363, 111-116 (2002) 111, 115

[57] X. Y. Xu, R. Ray, Y. L. Gu, H. J. Ploehn, L. Gearheart, K. Raker, W. A. Scrivens: Electrophoretic analysis and purification of fluorescent singlewalled carbon nanotube fragments, J. Am. Chem. Soc. 126, 12736-12737 (2004) 111, 115

[58] A. A. Vetcher, S. Srinivasan, I. A. Vetcher, S. M. Abramov, M. Kozlov, R. H. Baughman, S. D. Levene: Fractionation of SWNT/nucleic acid complexes by agarose gel electrophoresis, Nanotechnol. 17, 4263-4269 (2006) 111, 115

[59] R. Krupke, F. Hennrich, H. von Lohneysen, M. M. Kappes: Separation of metallic from semiconducting single-walled carbon nanotubes, Science 301, 344-347 (2003) 110, 111, 114, 135

[60] R. Krupke, F. Hennrich, H. B. Weber, M. M. Kappes, H. von Lohneysen: Simultaneous deposition of metallic bundles of single-walled carbon nanotubes using ac-dielectrophoresis, Nano Lett. 3, 1019-1023 (2003) 111, 135, 136

[61] R. Krupke, F. Hennrich, M. M. Kappes, H. V. Lohneysen: Surface conductance induced dielectrophoresis of semiconducting single-walled carbon nanotubes, Nano Lett. 4, 1395-1399 (2004) 111, 113, 135

[62] D. S. Lee, D. W. Kim, H. S. Kim, S. W. Lee, S. H. Jhang, Y. W. Park, E. E. B. Campbell: Extraction of semiconducting CNTs by repeated dielectrophoretic filtering, Appl. Phys. A-Mater. 80, 5-8 (2005) 111, 113

[63] Z. B. Zhang, X. J. Liu, E. E. B. Campbell, S. L. Zhang: Alternating current dielectrophoresis of carbon nanotubes, J. Appl. Phys. 98, 056103 (2005) 111

[64] T. Lutz, K. J. Donovan: Macroscopic scale separation of metallic and semiconducting nanotubes by dielectrophoresis, Carbon 43, 2508-2513 (2005) 111, 113

[65] C. W. Marquardt, S. Blatt, F. Hennrich, H. V. Lohneysen, R. Krupke: Probing dielectrophoretic force fields with metallic carbon nanotubes, Appl. Phys. Lett. 89, 183117 (2006) 111

[66] Z. Chen, Z. Y. Wu, L. M. Tong, H. P. Pan, Z. F. Liu: Simultaneous dielectrophoretic separation and assembly of single-walled carbon nanotubes on multigap nanoelectrodes and their thermal sensing properties, Anal. Chem. 78, 8069-8075 (2006) 111

[67] R. Krupke, S. Linden, M. Rapp, F. Hennrich: Thin films of metallic carbon nanotubes prepared by dielectrophoresis, Adv. Mater. 18, 1468-1470 (2006) 111,113 
[68] D. Chattopadhyay, L. Galeska, F. Papadimitrakopoulos: A route for bulk separation of semiconducting from metallic single-wall carbon nanotubes, J. Am. Chem. Soc. 125, 3370-3375 (2003) 111, 116

[69] G. G. Samsonidze, S. G. Chou, A. P. Santos, V. W. Brar, G. Dresselhaus, M. S. Dresselhaus, A. Selbst, A. K. Swan, M. S. Unlu, B. B. Goldberg, D. Chattopadhyay, S. N. Kim, F. Papadimitrakopoulos: Quantitative evaluation of the octadecylamine-assisted bulk separation of semiconducting and metallic single-wall carbon nanotubes by resonance raman spectroscopy, Appl. Phys. Lett. 85, 1006-1008 (2004) 111, 116

[70] Y. Maeda, S. Kimura, M. Kanda, Y. Hirashima, T. Hasegawa, T. Wakahara, Y. F. Lian, T. Nakahodo, T. Tsuchiya, T. Akasaka, J. Lu, X. W. Zhang, Z. X. Gao, Y. P. Yu, S. Nagase, S. Kazaoui, N. Minami, T. Shimizu, H. Tokumoto, R. Saito: Large-scale separation of metallic and semiconducting singlewalled carbon nanotubes, J. Am. Chem. Soc. 127, 10287-10290 (2005) 111, 116

[71] Y. Maeda, M. Kanda, M. Hashimoto, T. Hasegawa, S. Kimura, Y. F. Lian, T. Wakahara, T. Akasaka, S. Kazaoui, N. Minami, T. Okazaki, Y. Hayamizu, K. Hata, J. Lu, S. Nagase: Dispersion and separation of small-diameter singlewalled carbon nanotubes, J. Am. Chem. Soc. 128, 12239-12242 (2006) 111, 116

[72] K. J. Ziegler, D. J. Schmidt, U. Rauwald, K. N. Shah, E. L. Flor, R. H. Hauge, R. E. Smalley: Length-dependent extraction of single-walled carbon nanotubes, Nano Lett. 5, 2355-2359 (2005) 111, 115

[73] L. J. Li, A. N. Khlobystov, J. G. Wiltshire, G. A. D. Briggs, R. J. Nicholas: Diameter-selective encapsulation of metallocenes in single-walled carbon nanotubes, Nature Mater. 4, 481-485 (2005) 111

[74] A. Ortiz-Acevedo, H. Xie, V. Zorbas, W. M. Sampson, A. B. Dalton, R. H. Baughman, R. K. Draper, I. H. Musselman, G. R. Dieckmann: Diameter-selective solubilization of single-walled carbon nanotubes by reversible cyclic peptides, J. Am. Chem. Soc. 127, 9512-9517 (2005) 111, 116

[75] Z. H. Chen, X. Du, M. H. Du, C. D. Rancken, H. P. Cheng, A. G. Rinzler: Bulk separative enrichment in metallic or semiconducting single-walled carbon nanotubes, Nano Lett. 3, 1245-1249 (2003) 111, 116

[76] L. Kavan, L. Dunsch: Diameter-selective electrochemical doping of HiPco single-walled carbon nanotubes, Nano Lett. 3, 969-972 (2003) 111, 116

[77] A. Kukovecz, T. Pichler, R. Pfeiffer, H. Kuzmany: Diameter selective charge transfer in $\mathrm{p}$ - and n-doped single wall carbon nanotubes synthesized by the HiPCO method, Chem. Commun. pp. 1730-1731 (2002) 111, 116

[78] A. Kukovecz, T. Pichler, R. Pfeiffer, C. Kramberger, H. Kuzmany: Diameter selective doping of single wall carbon nanotubes, Phys. Chem. Chem. Phys. 5, 582-587 (2003) 111

[79] H. P. Li, B. Zhou, Y. Lin, L. R. Gu, W. Wang, K. A. S. Fernando, S. Kumar, L. F. Allard, Y. P. Sun: Selective interactions of porphyrins with semiconducting single-walled carbon nanotubes, J. Am. Chem. Soc. 126, 1014-1015 (2004) 111, 116

[80] M. S. Arnold, S. I. Stupp, M. C. Hersam: Enrichment of single-walled carbon nanotubes by diameter in density gradients, Nano Lett. 5, 713-718 (2005) 111,116 
[81] C. Menard-Moyon, N. Izard, E. Doris, C. Mioskowski: Separation of semiconducting from metallic carbon nanotubes by selective functionalization with azomethine ylides, J. Am. Chem. Soc. 128, 6552-6553 (2006) 111, 116

[82] S. Banerjee, S. S. Wong: Selective metallic tube reactivity in the solutionphase osmylation of single-walled carbon nanotubes, J. Am. Chem. Soc. 126, 2073-2081 (2004) 111

[83] L. An, Q. A. Fu, C. G. Lu, J. Liu: A simple chemical route to selectively eliminate metallic carbon nanotubes in nanotube network devices, J. Am. Chem. Soc. 126, 10520-10521 (2004) 111

[84] Y. Miyata, Y. Maniwa, H. Kataura: Selective oxidation of semiconducting single-wall carbon nanotubes by hydrogen peroxide, J. Phys. Chem. B 110, 25-29 (2006) 111

[85] A. Hassanien, M. Tokumoto, P. Umek, D. Vrbanic, M. Mozetic, D. Mihailovic, P. Venturini, S. Pejovnik: Selective etching of metallic single-wall carbon nanotubes with hydrogen plasma, Nanotechnol. 16, 278-281 (2005) 111

[86] G. Y. Zhang, P. F. Qi, X. R. Wang, Y. R. Lu, X. L. Li, R. Tu, S. Bangsaruntip, D. Mann, L. Zhang, H. J. Dai: Selective etching of metallic carbon nanotubes by gas-phase reaction, Science 314, 974-977 (2006) 111

[87] E. Borowiak-Palen, T. Pichler, X. Liu, M. Knupfer, A. Graff, O. Jost, W. Pompe, R. J. Kalenczuk, J. Fink: Reduced diameter distribution of singlewall carbon nanotubes by selective oxidation, Chem. Phys. Lett. 363, 567-572 (2002) 111

[88] E. Menna, F. D. Negra, M. D. Fontana, M. Meneghetti: Selectivity of chemical oxidation attack of single-wall carbon nanotubes in solution, Phys. Rev. B 68, 193412 (2003) 111

[89] P. C. Collins, M. S. Arnold, P. Avouris: Engineering carbon nanotubes and nanotube circuits using electrical breakdown, Science 292, 706-709 (2001) 111,115

[90] R. Seidel, A. P. Graham, E. Unger, G. S. Duesberg, M. Liebau, W. Steinhoegl, F. Kreupl, W. Hoenlein: High-current nanotube transistors, Nano Lett. 4, 831-834 (2004) 111, 115

[91] H. Q. Peng, N. T. Alvarez, C. Kittrell, R. H. Hauge, H. K. Schmidt: Dielectrophoresis field flow fractionation of single-walled carbon nanotubes, J. Am. Chem. Soc. 128, 8396-8397 (2006) 112, 113

[92] S. N. Kim, Z. T. Luo, F. Papadimitrakopoulos: Diameter and metallicity dependent redox influences on the separation of single-wall carbon nanotubes, Nano Lett. 5, 2500-2504 (2005) 112, 116

[93] M. S. Arnold, A. A. Green, J. F. Hulvat, S. I. Stupp, M. C. Hersam: Sorting carbon nanotubes by electronic structure using density differentiation, Nature Nanotechnol. 1, 60-65 (2006) 112, 114, 116

[94] L. J. Li, R. J. Nicholas: Bandgap-selective chemical doping of semiconducting single-walled carbon nanotubes, Nanotechnol. 15, 1844-1847 (2004) 112

[95] M. Zheng, A. Jagota, E. D. Semke, B. A. Diner, R. S. Mclean, S. R. Lustig, R. E. Richardson, N. G. Tassi: DNA-assisted dispersion and separation of carbon nanotubes, Nature Mater. 2, 338-342 (2003) 112, 113, 114, 116

[96] M. Zheng, A. Jagota, M. S. Strano, A. P. Santos, P. Barone, S. G. Chou, B. A. Diner, M. S. Dresselhaus, R. S. McLean, G. B. Onoa, G. G. Samsonidze, E. D. Semke, M. Usrey, D. J. Walls: Structure-based carbon nanotube sorting 
by sequence-dependent DNA assembly, Science 302, 1545-1548 (2003) 112, 114,116

[97] M. S. Strano, M. Zheng, A. Jagota, G. B. Onoa, D. A. Heller, P. W. Barone, M. L. Usrey: Understanding the nature of the DNA-assisted separation of single-walled carbon nanotubes using fluorescence and raman spectroscopy, Nano Lett. 4, 543-550 (2004) 112, 116

[98] S. R. Lustig, A. Jagota, C. Khripin, M. Zheng: Theory of structure-based carbon nanotube separations by ion-exchange chromatography of DNA/CNT hybrids, J. Phys. Chem. B 109, 2559-2566 (2005) 112, 116

[99] M. S. Strano, C. B. Huffman, V. C. Moore, M. J. O'Connell, E. H. Haroz, J. Hubbard, M. Miller, K. Rialon, C. Kittrell, S. Ramesh, R. H. Hauge, R. E. Smalley: Reversible, band-gap-selective protonation of single-walled carbon nanotubes in solution, J. Phys. Chem. B 107, 6979-6985 (2003) 112

[100] M. S. Strano, C. A. Dyke, M. L. Usrey, P. W. Barone, M. J. Allen, H. W. Shan, C. Kittrell, R. H. Hauge, J. M. Tour, R. E. Smalley: Electronic structure control of single-walled carbon nanotube functionalization, Science 301, 15191522 (2003) 112, 113, 114, 116

[101] M. L. Usrey, E. S. Lippmann, M. S. Strano: Evidence for a two-step mechanism in electronically selective single-walled carbon nanotube reactions, J. Am. Chem. Soc. 127, 16129-16135 (2005) 112

[102] C. J. Wang, Q. Cao, T. Ozel, A. Gaur, J. A. Rogers, M. Shim: Electronically selective chemical functionalization of carbon nanotubes: Correlation between Raman spectral and electrical responses, J. Am. Chem. Soc. 127, 1146011468 (2005) 112

[103] C. A. Dyke, M. P. Stewart, J. M. Tour: Separation of single-walled carbon nanotubes on silica gel. Materials morphology and Raman excitation wavelength affect data interpretation, J. Am. Chem. Soc. 127, 4497-4509 (2005) $112,113,116$

[104] S. Baik, M. Usrey, L. Rotkina, M. Strano: Using the selective functionalization of metallic single-walled carbon nanotubes to control dielectrophoretic mobility, J. Phys. Chem. B 108, 15560-15564 (2004) 112, 113

[105] M. S. Strano: Probing chiral selective reactions using a revised Kataura plot for the interpretation of single-walled carbon nanotube spectroscopy, J. Am. Chem. Soc. 125, 16148-16153 (2003) 112, 116

[106] K. Kamaras, M. E. Itkis, H. Hu, B. Zhao, R. C. Haddon: Covalent bond formation to a carbon nanotube metal, Science 301, 1501-1501 (2003) 112

[107] K. H. An, J. S. Park, C. M. Yang, S. Y. Jeong, S. C. Lim, C. Kang, J. H. Son, M. S. Jeong, Y. H. Lee: A diameter-selective attack of metallic carbon nanotubes by nitronium ions, J. Am. Chem. Soc. 127, 5196-5203 (2005) 112

[108] C. M. Yang, J. S. Park, K. H. An, S. C. Lim, K. Seo, B. Kim, K. A. Park, S. Han, C. Y. Park, Y. H. Lee: Selective removal of metallic single-walled carbon nanotubes with small diameters by using nitric and sulfuric acids, J. Phys. Chem. B 109, 19242-19248 (2005) 112

[109] C. M. Yang, K. H. An, J. S. Park, K. A. Park, S. C. Lim, S. H. Cho, Y. S. Lee, W. Park, C. Y. Park, Y. H. Lee: Preferential etching of metallic single-walled carbon nanotubes with small diameter by fluorine gas, Phys. Rev. B 73, 075419 (2006) 112 
[110] T. W. Odom, J. L. Huang, P. Kim, C. M. Lieber: Structure and electronic properties of carbon nanotubes, J. Phys. Chem. B 104, 2794-2809 (2000) 112,116

[111] E. Joselevich: Chemistry and electronics of carbon nanotubes go together, Angew. Chem. Int. Ed. 43, 2992-2994 (2004) 116

[112] E. Joselevich: Electronic structure and chemical reactivity of carbon nanotubes: A chemist's view, Chem. Phys. Chem. 5, 619-624 (2004) 116

[113] Z. F. Chen, W. Thiel, A. Hirsch: Reactivity of the convex and concave surfaces of single-walled carbon nanotubes (SWCNTs) towards addition reactions: Dependence on the carbon-atom pyramidalization, Chem. Phys. Chem. 4, 93-97 (2003) 116

[114] R. E. Smalley, Y. B. Li, V. C. Moore, B. K. Price, R. Colorado, H. K. Schmidt, R. H. Hauge, A. R. Barron, J. M. Tour: Single wall carbon nanotube amplification: En route to a type-specific growth mechanism, J. Am. Chem. Soc. 128, 15824-15829 (2006) 116, 142

[115] Y. H. Wang, M. J. Kim, H. W. Shan, C. Kittrell, H. Fan, L. M. Ericson, W. F. Hwang, S. Arepalli, R. H. Hauge, R. E. Smalley: Continued growth of single-walled carbon nanotubes, Nano Lett. 5, 997-1002 (2005) 116, 118, 142

[116] M. J. Kim, E. Haroz, Y. Wang, H. Shan, N. Nicholas, C. Kittrell, V. C. Moore, Y. Jung, D. Luzzi, R. Wheeler, T. BensonTolle, H. Fan, S. Da, W. F. Hwang, T. J. Wainerdi, H. Schmidt, R. H. Hauge, R. E. Smalley: Nanoscopically flat open-ended single-walled carbon nanotube substrates for continued growth, Nano Lett. 7, 15-21 (2007) 116, 142

[117] M. Hamm, J. A. Elliott, H. J. Smithson, A. H. Windle: Mater. Res. Soc. Symp. Proc. 788 (2004) 117

[118] S. Giordani, S. D. Bergin, V. Nicolosi, S. Lebedkin, M. M. Kappes, W. J. Blau, J. N. Coleman: Debundling of single-walled nanotubes by dilution: Observation of large populations of individual nanotubes in amide solvent dispersions, J. Phys. Chem. B. 110, 15708-15718 (2006) 118

[119] M. S. P. Shaffer, X. Fan, A. H. Windle: Dispersion and packing of carbon nanotubes, Carbon 36, 1603-1612 (1998) 118

[120] P. J. Flory, P. R. Soc.: Phase equilibria in solutions of rod-like particles, London A 234, 73-89 (1956) 118

[121] W. H. Song, I. A. Kinloch, A. H. Windle: Nematic liquid crystallinity of multiwall carbon nanotubes, Science 302, 1363-1363 (2003) 118

[122] S. J. Zhang, I. A. Kinloch, A. H. Windle: Mesogenicity drives fractionation in lyotropic aqueous suspensions of multiwall carbon nanotubes, Nano Lett. 6, 568-572 (2006) 119

[123] H. G. Chae, S. Kumar: Rigid-rod polymeric fibers, J. Appl. Polym. Sci. 100, 791-802 (2006) 119

[124] L. M. Ericson, H. Fan, H. Q. Peng, V. A. Davis, W. Zhou, J. Sulpizio, Y. H. Wang, R. Booker, J. Vavro, C. Guthy, A. N. G. Parra-Vasquez, M. J. Kim, S. Ramesh, R. K. Saini, C. Kittrell, G. Lavin, H. Schmidt, W. W. Adams, W. E. Billups, M. Pasquali, W. F. Hwang, R. H. Hauge, J. E. Fischer, R. E. Smalley: Macroscopic, neat, single-walled carbon nanotube fibers, Science 305, 1447-1450 (2004) 119, 120 
[125] J. Steinmetz, M. Glerup, M. Paillet, P. Bernier, M. Holzinger: Production of pure nanotube fibers using a modified wet-spinning method, Carbon 43, 2397-2400 (2005) 120

[126] S. Zhang, K. K. K. Koziol, I. A. Kinloch, A. H. Windle: Carbon (2007) submitted 120

[127] A. Penicaud, L. Valat, A. Derre, P. Poulin, C. Zakri, O. Roubeau, M. Maugey, P. Miaudet, E. Anglaret, P. Petit, A. Loiseau, S. Enouz: Mild dissolution of carbon nanotubes: Composite carbon nanotube fibres from polyelectrolyte solutions, Compos. Sci. Technol. 67, 795-797 (2007) 120, 121

[128] L. X. Zheng, M. J. O'Connell, S. K. Doorn, X. Z. Liao, Y. H. Zhao, E. A. Akhadov, M. A. Hoffbauer, B. J. Roop, Q. X. Jia, R. C. Dye, D. E. Peterson, S. M. Huang, J. Liu, Y. T. Zhu: Ultralong single-wall carbon nanotubes, Nature Mater. 3, 673-676 (2004) 120, 123

[129] B. Vigolo, A. Penicaud, C. Coulon, C. Sauder, R. Pailler, C. Journet, P. Bernier, P. Poulin: Macroscopic fibers and ribbons of oriented carbon nanotubes, Science 290, 1331-1334 (2000) 120

[130] P. Poulin, B. Vigolo, P. Launois: Films and fibers of oriented single wall nanotubes, Carbon 40, 1741-1749 (2002) 120, 121

[131] H. H. Gommans, J. W. Alldredge, H. Tashiro, J. Park, J. Magnuson, A. G. Rinzler: Fibers of aligned single-walled carbon nanotubes: Polarized Raman spectroscopy, J. Appl. Phys. 88, 2509-2514 (2000) 121

[132] B. Vigolo, P. Poulin, M. Lucas, P. Launois, P. Bernier: Improved structure and properties of single-wall carbon nanotube spun fibers, Appl. Phys. Lett. 81, 1210-1212 (2002) 121

[133] P. Miaudet, S. Badaire, M. Maugey, A. Derre, V. Pichot, P. Launois, P. Poulin, C. Zakri: Hot-drawing of single and multiwall carbon nanotube fibers for high toughness and alignment, Nano Lett. 5, 2212-2215 (2005) 121,122

[134] V. Pichot, S. Badaire, P. A. Albouy, C. Zakri, P. Poulin, P. Launois: Structural and mechanical properties of single-wall carbon nanotube fibers, Phys. Rev. B 74, 245416 (2006) 121

[135] A. B. Dalton, S. Collins, E. Munoz, J. M. Razal, V. H. Ebron, J. P. Ferraris, J. N. Coleman, B. G. Kim, R. H. Baughman: Super-tough carbon-nanotube fibres - These extraordinary composite fibres can be woven into electronic textiles, Nature 423, 703-703 (2003) 121

[136] A. Penicaud, P. Poulin, A. Derre, E. Anglaret, P. Petit: Spontaneous dissolution of a single-wall carbon nanotube salt, J. Am. Chem. Soc. 127, 8-9 (2005) 121

[137] T. V. Sreekumar, T. Liu, B. G. Min, H. Guo, S. Kumar, R. H. Hauge, R. E. Smalley: Polyacrylonitrile single-walled carbon nanotube composite fibers, Adv. Mater. 16, 58-61 (2004) 122

[138] C. Y. Wang, V. Mottaghitalab, C. O. Too, G. M. Spinks, G. G. Wallace: Polyaniline and polyaniline-carbon nanotube composite fibres as battery materials in ionic liquid electrolyte, J. Power Sources 163, 1105-1109 (2007) 122

[139] B. G. Min, T. V. Sreekumar, T. Uchida, S. Kumar: Oxidative stabilization of PAN/SWNT composite fiber, Carbon 43, 599-604 (2005) 122 
[140] Y. P. Wang, R. L. Cheng, L. L. Liang, Y. M. Wang: Study on the preparation and characterization of ultra-high molecular weight polyethylene-carbon nanotubes composite fiber, Compos. Sci. Technol. 65, 793-797 (2005) 122

[141] K. L. Jiang, Q. Q. Li, S. S. Fan: Nanotechnology: Spinning continuous carbon nanotube yarns - Carbon nanotubes weave their way into a range of imaginative macroscopic applications, Nature 419, 801-801 (2002) 122

[142] M. Zhang, K. R. Atkinson, R. H. Baughman: Multifunctional carbon nanotube yarns by downsizing an ancient technology, Science 306, 1358-1361 (2004) 122, 123

[143] J. W. S. Hearle, P. Grosberg, S. Becker: Structural Mechanics of Fibres, Yarns and Fabrics (Wiley, New York 1969) 122

[144] Q. Li, X. Zhang, R. F. DePaula, L. X. Zheng, Y. H. Zhao, L. Stan, T. G. Holesinger, P. N. Arendt, D. E. Peterson, Y. T. Zhu: Poster presentation preprint, MRS Fall Meeting, Boston (2006) 123

[145] M. Zhang, S. L. Fang, A. A. Zakhidov, S. B. Lee, A. E. Aliev, C. D. Williams, K. R. Atkinson, R. H. Baughman: Strong, transparent, multifunctional, carbon nanotube sheets, Science 309, 1215-1219 (2005) 123

[146] Y. L. Li, I. A. Kinloch, A. H. Windle: Direct spinning of carbon nanotube fibers from chemical vapor deposition synthesis, Science 304, 276-278 (2004) 123

[147] H. W. Zhu, C. L. Xu, D. H. Wu, B. Q. Wei, R. Vajtai, P. M. Ajayan: Direct synthesis of long single-walled carbon nanotube strands, Science 296, 884-886 (2002) 123

[148] M. Motta, A. Moisala, I. A. Kinloch, V. Premnath, M. Pick, A. H. Windle: The parameter space for the direct spinning of fibres and films of carbon nanotubes, Physica E 37, 40-43 (2007) 124

[149] M. Motta, Y. L. Li, I. Kinloch, A. Windle: Mechanical properties of continuously spun fibers of carbon nanotubes, Nano Lett. 5, 1529-1533 (2005) 124

[150] M. Motta, A. Moisala, I. Kinloch, A. H. Windle: High performance fibres from dog bone carbon nanotubes, Adv. Mater. 19, 3721-3726 (2007) 124

[151] R. F. Service: Assembling nanocircuits from the bottom up, Science 293, 782-785 (2001) 126

[152] S. M. Huang, L. M. Dai, A. W. H. Mau: Patterned growth and contact transfer of well-aligned carbon nanotube films, J. Phys. Chem. B. 103, 4223-4227 (1999) 126

[153] B. Q. Wei, R. Vajtai, Y. Jung, J. Ward, R. Zhang, G. Ramanath, P. M. Ajayan: Organized assembly of carbon nanotubes - Cunning refinements help to customize the architecture of nanotube structures, Nature 416, 495-496 (2002) 126

[154] L. M. Dai, A. Patil, X. Y. Gong, Z. X. Guo, L. Q. Liu, Y. Liu, D. B. Zhu: Aligned nanotubes, Chem. Phys. Chem. 4, 1150-1169 (2003) 126

[155] Z. F. Ren, Z. P. Huang, J. W. Xu, J. H. Wang: Synthesis of large arrays of well-aligned carbon nanotubes on glass, Science 282, 1105-1107 (1998) 126

[156] S. Fan, M. Chapline, N. Franklin, T. Tombler, A. Cassell, H. Dai: Self-oriented regular arrays of carbon nanotubes and their field emission properties, Science 283, 512-514 (1999) 126 
[157] K. Hata, D. N. Futaba, K. Mizuno, T. Namai, M. Yumura, S. Iijima: Waterassisted highly efficient synthesis of impurity-free single-waited carbon nanotubes, Science 306, 1362-1364 (2004) 127, 128

[158] Y. H. Yun, V. Shanov, Y. Tu, S. Subramaniam, M. J. Schulz: Growth mechanism of long aligned multiwall carbon nanotube arrays by water-assisted chemical vapor deposition, J. Phys. Chem. B. 110, 23920-23925 (2006) 127

[159] Q. W. Li, X. F. Zhang, R. F. DePaula, L. X. Zheng, Y. H. Zhao, L. Stan, T. G. Holesinger, P. N. Arendt, D. E. Peterson, Y. T. T. Zhu: Sustained growth of ultralong carbon nanotube arrays for fiber spinning, Adv. Mater. 18, 3160-3163 (2006) 127

[160] H. Ago, N. Uehara, N. Yoshihara, M. Tsuji, M. Yumura, N. Tomonaga, T. Setoguchi: Gas analysis of the CVD process for high yield growth of carbon nanotubes over metal-supported catalysts, Carbon 44, 2912-2918 (2006) 127

[161] S. Chakrabarti, T. Nagasaka, Y. Yoshikawa, L. J. Pan, Y. Nakayama: Growth of super long aligned brush-like carbon nanotubes, Jpn. J. Appl. Phys. 2 45, L720-L722 (2006) 127

[162] D. N. Futaba, K. Hata, T. Namai, T. Yamada, K. Mizuno, Y. Hayamizu, M. Yumura, S. Iijima: $84 \%$ Catalyst activity of water-assisted growth of single walled carbon nanotube forest characterization by a statistical and macroscopic approach, J. Phys. Chem. B 110, 8035-8038 (2006) 127

[163] G. F. Zhong, T. Iwasaki, H. Kawarada: Semi-quantitative study on the fabrication of densely packed and vertically aligned single-walled carbon nanotubes, Carbon 44, 2009-2014 (2006) 127

[164] D. N. Futaba, K. Hata, T. Yamada, K. Mizuno, M. Yumura, S. Iijima: Kinetics of water-assisted single-walled carbon nanotube synthesis revealed by a timeevolution analysis, Phys. Rev. Lett. 95, 056104 (2005) 128, 130

[165] T. Yamada, T. Namai, K. Hata, D. N. Futaba, K. Mizuno, J. Fan, M. Yudasaka, M. Yumura, S. Iijima: Size-selective growth of double-walled carbon nanotube forests from engineered iron catalysts, Nature Nanotechnol. 1, 131136 (2006) 130, 131

[166] T. Hiraoka, T. Yamada, K. Hata, D. N. Futaba, H. Kurachi, S. Uemura, M. Yumura, S. Iijima: Synthesis of single- and double-walled carbon nanotube forests on conducting metal foils, J. Am. Chem. Soc. 128, 13338-13339 (2006) 131,132

[167] D. N. Futaba, K. Hata, T. Yamada, T. Hiraoka, Y. Hayamizu, Y. Kakudate, O. Tanaike, H. Hatori, M. Yumura, S. Iijima: Shape-engineerable and highly densely packed single-walled carbon nanotubes and their application as supercapacitor electrodes, Nature Mater. 5, 987-994 (2006) 132, 133, 134

[168] Y. H. Yan, M. B. Chan-Park, Q. Zhang: Advances in carbon-nanotube assembly, Small 3, 24-42 (2007) 133

[169] J. Liu, M. J. Casavant, M. Cox, D. A. Walters, P. Boul, W. Lu, A. J. Rimberg, K. A. Smith, D. T. Colbert, R. E. Smalley: Controlled deposition of individual single-walled carbon nanotubes on chemically functionalized templates, Chem. Phys. Lett. 303, 125-129 (1999) 133

[170] C. Klinke, J. B. Hannon, A. Afzali, P. Avouris: Field-Effect Transistors Assembled from Functionalized Carbon Nanotubes, Nano Lett. 6, 906-910 (2006) 133, 134

[171] S. G. Rao, L. Huang, W. Setyawan, S. H. Hong: Large-scale assembly of carbon nanotubes, Nature 425, 36-37 (2003) 133, 135 
[172] Y. H. Wang, D. Maspoch, S. L. Zou, G. C. Schatz, R. E. Smalley, C. A. Mirkin: Controlling the shape, orientation, and linkage of carbon nanotube features with nano affinity templates, Proc. Nat. Acad. Sci. 103, 2026-2031 (2006) 133

[173] S. Zou, D. Maspoch, Y. Wang, C. A. Mirkin, G. C. Schatz: Rings of Single-Walled Carbon Nanotubes: Molecular-Template Directed Assembly and Monte Carlo Modeling, Nano Lett. 7, 276-280 (2007) 133

[174] A. L. Bassi, M. G. Beghi, C. S. Casari, C. E. Bottani, A. Podesta, P. Milani, A. Zakhidov, R. Baughman, D. A. Walters, R. E. Smalley: Inelastic light scattering from magnetically aligned single-walled carbon nanotubes and estimate of their two-dimensional Young's modulus, Diam. Relat. Mater. 12, 806-810 (2003) 135

[175] M. J. Casavant, D. A. Walters, J. J. Schmidt, R. E. Smalley: Neat macroscopic membranes of aligned carbon nanotubes, J. Appl. Phys. 93, 2153-2156 (2003) 135

[176] J. E. Fischer, W. Zhou, J. Vavro, M. C. Llaguno, C. Guthy, R. Haggenmueller, M. J. Casavant, D. E. Walters, R. E. Smalley: Magnetically aligned single wall carbon nanotube films: Preferred orientation and anisotropic transport properties, J. Appl. Phys. 93, 2157-2163 (2003) 135

[177] D. A. Walters, M. J. Casavant, X. C. Qin, C. B. Huffman, P. J. Boul, L. M. Ericson, E. H. Haroz, M. J. O'Connell, K. Smith, D. T. Colbert, R. E. Smalley: In-plane-aligned membranes of carbon nanotubes, Chem. Phys. Lett. 338, 14-20 (2001) 135

[178] B. W. Smith, Z. Benes, D. E. Luzzi, J. E. Fischer, D. A. Walters, M. J. Casavant, J. Schmidt, R. E. Smalley: Structural anisotropy of magnetically aligned single wall carbon nanotube films, Appl. Phys. Lett. 77, 663-665 (2000) 135

[179] M. D. Lay, J. P. Novak, E. S. Snow: Simple route to large-scale ordered arrays of liquid-deposited carbon nanotubes, Nano Lett. 4, 603-606 (2004) 135

[180] J. Q. Li, Q. Zhang, N. Peng, Q. Zhu: Manipulation of carbon nanotubes using AC dielectrophoresis, Appl. Phys. Lett. 86, 153116 (2005) 135

[181] J. Tang, G. Yang, Q. Zhang, A. Parhat, B. Maynor, J. Liu, L. C. Qin, O. Zhou: Rapid and reproducible fabrication of carbon nanotube AFM probes by dielectrophoresis, Nano Lett. 5, 11-14 (2005) 135

[182] J. Tang, B. Gao, H. Z. Geng, O. D. Velev, L. C. Qin, O. Zhou: Assembly of ID nanostructures into sub-micrometer diameter fibrils with controlled and variable length by dielectrophoresis, Adv. Mater. 15, 1352-1355 (2003) 135

[183] R. Krupke, F. Hennrich, H. B. Weber, D. Beckmann, O. Hampe, S. Malik, M. M. Kappes, H. V. Lohneysen: Contacting single bundles of carbon nanotubes with alternating electric fields, Appl. Phys. A-Mater. 76, 397-400 (2003) 135

[184] X. Li, L. Zhang, X. Wang, I. Shimoyama, X. Sun, W.-S. Seo, H. Dai: Langmuir-Blodgett assembly of densely aligned single-walled carbon nanotubes from bulk materials, J. Am. Chem. Soc. 129, 4890-4891 (2007) 135, 136,137

[185] J. Kong, H. T. Soh, A. M. Cassell, C. F. Quate, H. J. Dai: Synthesis of individual single-walled carbon nanotubes on patterned silicon wafers, Nature 395, 878-881 (1998) 137

[186] E. Joselevich, C. M. Lieber: Vectorial growth of metallic and semiconducting single-wall carbon nanotubes, Nano Lett. 2, 1137-1141 (2002) 137, 138 
[187] L. X. Benedict, S. G. Louie, M. L. Cohen: Static Polarizabilities of Single-Wall Carbon Nanotubes, Phys. Rev. B 52, 8541-8549 (1995) 137

[188] Y. G. Zhang, A. L. Chang, J. Cao, Q. Wang, W. Kim, Y. M. Li, N. Morris, E. Yenilmez, J. Kong, H. J. Dai: Electric-field-directed growth of aligned single-walled carbon nanotubes, Appl. Phys. Lett. 79, 3155-3157 (2001) 137, 138

[189] A. Ural, Y. M. Li, H. J. Dai: Electric-field-aligned growth of single-walled carbon nanotubes on surfaces, Appl. Phys. Lett. 81, 3464-3466 (2002) 137

[190] A. Nojeh, A. Ural, R. F. Pease, H. J. Dai: Electric-field-directed growth of carbon nanotubes in two dimensions, J. Vac. Sci. Technol. B 22, 3421-3425 (2004) 139

[191] A. Ismach, E. Joselevich: Orthogonal self-assembly of carbon nanotube crossbar architectures by simultaneous graphoepitaxy and field-directed growth, Nano Lett. 6, 1706-1710 (2006) 139, 145, 147

[192] S. Dittmer, J. Svensson, E. E. B. Campbell: Electric field aligned growth of single-walled carbon nanotubes, Curr. Appl. Phys. 4, 595-598 (2004) 139

[193] I. Radu, Y. Hanein, D. H. Cobden: Oriented growth of single-wall carbon nanotubes using alumina patterns, Nanotechnol. 15, 473-476 (2004) 139

[194] C. Kocabas, M. A. Meitl, A. Gaur, M. Shim, J. A. Rogers: Aligned arrays of single-walled carbon nanotubes generated from random networks by orientationally selective laser ablation, Nano Lett. 4, 2421-2426 (2004) 139

[195] K. H. Lee, J. M. Cho, W. Sigmund: Control of growth orientation for carbon nanotubes, Appl. Phys. Lett. 82, 448-450 (2003) 139

[196] N. Kumar, W. Curtis, J. I. Hahm: Laterally aligned, multiwalled carbon nanotube growth using Magnetospirillium magnetotacticum, Appl. Phys. Lett. 86, $173101(2005) 139$

[197] S. M. Huang, X. Y. Cai, J. Liu: Growth of millimeter-long and horizontally aligned single-walled carbon nanotubes on flat substrates, J. Am. Chem. Soc. 125, 5636-5637 (2003) 139, 140

[198] S. M. Huang, Q. Fu, L. An, J. Liu: Growth of aligned SWNT arrays from water-soluble molecular clusters for nanotube device fabrication, Phys. Chem. Chem. Phys. 6, 1077-1079 (2004) 139

[199] S. M. Huang, B. Maynor, X. Y. Cai, J. Liu: Ultralong, well-aligned singlewalled carbon nanotube architectures on surfaces, Adv. Mater. 15, 1651-1655 (2003) 139

[200] S. M. Huang, X. Y. Cai, C. S. Du, J. Liu: Oriented long single walled carbon nanotubes on substrates from floating catalysts, J. Phys. Chem. B 107, 13251-13254 (2003) 139

[201] S. D. Li, Z. Yu, C. Rutherglen, P. J. Burke: Electrical properties of $0.4 \mathrm{~cm}$ long single-walled carbon nanotubes, Nano Lett. 4, 2003-2007 (2004) 141

[202] Z. Yu, S. D. Li, P. J. Burke: Synthesis of aligned arrays of millimeter long, straight single-walled carbon nanotubes, Chem. Mater. 16, 3414-3416 (2004) 141

[203] B. H. Hong, J. Y. Lee, T. Beetz, Y. M. Zhu, P. Kim, K. S. Kim: Quasicontinuous growth of ultralong carbon nanotube arrays, J. Am. Chem. Soc. 127, 15336-15337 (2005) 141

[204] W. W. Zhou, Z. Y. Han, J. Y. Wang, Y. Zhang, Z. Jin, X. Sun, Y. W. Zhang, C. H. Yan, Y. Li: Copper catalyzing growth of single-walled carbon nanotubes on substrates, Nano Lett. 6, 2987-2990 (2006) 141 
[205] D. E. Hooks, T. Fritz, M. D. Ward: Epitaxy and molecular organization on solid substrates, Adv. Mater. 13, 227-241 (2001) 141

[206] A. Ismach, D. Kantorovich, E. Joselevich: Carbon nanotube graphoepitaxy: Highly oriented growth by faceted nanosteps, J. Am. Chem. Soc. 127, 1155411555 (2005) 142, 144, 145, 146

[207] M. Su, Y. Li, B. Maynor, A. Buldum, J. P. Lu, J. Liu: Lattice-oriented growth of single-walled carbon nanotubes, J. Phys. Chem. B 104, 6505-6508 (2000) 142

[208] M. Tominaga, A. Ohira, A. Kubo, I. Taniguchi, M. Kunitake: Growth of carbon nanotubes on a gold(111) surface using two-dimensional iron oxide nano-particle catalysts derived from iron storage protein, Chem. Commun. pp. 1518-1519 (2004) 142

[209] V. Derycke, R. Martel, M. Radosvljevic, F. M. R. Ross, P. Avouris: Catalystfree growth of ordered single-walled carbon nanotube networks, Nano Lett. 2, 1043-1046 (2002) 142

[210] L. B. Ruppalt, P. M. Albrecht, J. W. Lyding: Atomic resolution scanning tunneling microscope study of single-wailed carbon nanotubes on GaAs(110), J. Vac. Sci. Technol. B. 22, 2005-2007 (2004) 142

[211] L. B. Ruppalt, P. M. Albrecht, J. W. Lyding: UHV-STM study of singlewalled carbon nanotubes applied to the GaAs(110) and InAs(110) surfaces, J. Phys. IV 132, 31-34 (2006) 142

[212] A. Ismach, L. Segev, E. Wachtel, E. Joselevich: Atomic-step-templated formation of single wall carbon nanotube patterns, Angew. Chem. Int. Ed. 43, 6140-6143 (2004) 142, 143, 144

[213] S. Han, X. L. Liu, C. W. Zhou: Template-free directional growth of singlewalled carbon nanotubes on a- and r-plane sapphire, J. Am. Chem. Soc. 127, 5294-5295 (2005) 142

[214] H. Ago, K. Nakamura, K. Ikeda, N. Uehara, N. Ishigami, M. Tsuji: Aligned growth of isolated single-walled carbon nanotubes programmed by atomic arrangement of substrate surface, Chem. Phys. Lett. 408, 433-438 (2005) 142

[215] H. Ago, N. Uehara, K. Ikeda, R. Ohdo, K. Nakamura, M. Tsuji: Synthesis of horizontally-aligned single-walled carbon nanotubes with controllable density on sapphire surface and polarized Raman spectroscopy, Chem. Phys. Lett. 421, 399-403 (2006) 142

[216] Q. K. Yu, G. T. Qin, H. Li, Z. H. Xia, Y. B. Nian, S. S. Pei: Mechanism of horizontally aligned growth of single-wall carbon nanotubes on R-plane sapphire, J. Phys. Chem. B 110, 22676-22680 (2006) 142

[217] X. L. Liu, S. Han, C. W. Zhou: Novel nanotube-on-insulator (NOI) approach toward single-walled carbon nanotube devices, Nano Lett. 6, 34-39 (2006) 143

[218] M. Maret, K. Hostache, M. C. Schouler, B. Marcus, F. Roussel-Dherbey, M. Albrecht, P. Gadelle: Oriented growth of single-walled carbon nanotubes on a $\mathrm{MgO}(001)$ surface, Carbon 45, 180-187 (2007) 143

[219] M. Souza, A. Jorio, C. Fantini, B. R. A. Neves, M. A. Pimenta, R. Saito, A. Ismach, E. Joselevich, V. W. Brar, G. G. Samsonidze, G. Dresselhaus, M. S. Dresselhaus: Single- and double-resonance Raman G-band processes in carbon nanotubes, Phys. Rev. B 69, 241403 (2004) 143 
[220] A. Ismach, D. Kantorovich, J. Berson, N. Geblinger, L. Segev, E. Wachtel, H. Son, A. Jorio, M. S. Dresselhaus, G. Dresselhaus, E. Joselevich: Epitaxial Modes of Carbon Nanotube Growth on Vicinal a-Al2O3 (0001) Surfaces, unpublished 143, 144

[221] C. Kocabas, S. H. Hur, A. Gaur, M. A. Meitl, M. Shim, J. A. Rogers: Guided growth of large-scale, horizontally aligned arrays of single-walled carbon nanotubes and their use in thin-film transistors, Small 1, 1110-1116 (2005) 143

[222] C. Kocabas, M. Shim, J. A. Rogers: Spatially selective guided growth of highcoverage arrays and random networks of single-walled carbon nanotubes and their integration into electronic devices, J. Am. Chem. Soc. 128, 4540-4541 (2006) 143

[223] H. I. Smith, D. C. Flanders: Oriented Crystal-Growth on Amorphous Substrates Using Artificial Surface-Relief Gratings, Appl. Phys. Lett. 32, 349-350 (1978) 144

[224] R. A. Segalman, H. Yokoyama, E. J. Kramer: Graphoepitaxy of spherical domain block copolymer films, Adv. Mater. 13, 1152-1155 (2001) 144

[225] T. Rueckes, K. Kim, E. Joselevich, G. Y. Tseng, C. L. Cheung, C. M. Lieber: Carbon nanotube-based nonvolatile random access memory for molecular computing, Science 289, 94-97 (2000) 145

[226] A. Javey, H. J. Dai: Regular arrays of $2 \mathrm{~nm}$ metal nanoparticles for deterministic synthesis of nanomaterials, J. Am. Chem. Soc. 127, 11942-11943 (2005) 147,148

[227] S. Helveg, C. Lopez-Cartes, J. Sehested, P. L. Hansen, B. S. Clausen, J. R. Rostrup-Nielsen, F. Abild-Pedersen, J. K. Norskov: Atomic-scale imaging of carbon nanofibre growth, Nature 427, 426-429 (2004) 148

\section{Index}

alignment, 144

arc discharge, 103

array, 141

assembly, 133

centrifugation, 111

chemical reactivity, 115

chemical vapor deposition, 103

chromatography, 111

Clausius-Mossotti factor, 113

covalent functionalization, 113

crossbar architecture, 144

dielectric constant, 113

dielectrophoresis, 110

DNA, 112

doping, 116

epitaxy, 141 graphoepitaxy, 144

lattice-directed, 142

ledge-directed, 142

nanotube epitaxy, 141

fiber, 116

field emission, 131

field-flow fractionation, 111

forest, 122, 126

growth, 102

atomic-step-templated, 142

catalyst-free, 141

continued, 116

epitaxial, 141

field-directed, 137, 144

flow-directed, 139, 144

horizontally aligned, 136

lattice-directed, 142 
organization, 101

patterned, 145

preparation, 102

production, 101

rate, 128

selective, 106

surface-directed, 141

synthesis, 101

vertically aligned, 126

kinks, 142

Langmuir-Blodgett (LB), 134

laser ablation, 103

laser vaporization, 103

liquid suspension, 118 mass production, 105

metrology, 109

patterning, 146

purification, 107

Raman spectroscopy, 136

selectivity, 109

separation, 110

sorting, 109

spinning, 119

stiffness, 119

strength, 119

yarn, 122 